S1 Supporting Information. Rossler, Hartgerink, Zerull, Anderson et. al.

\title{
Au(I)-Catalyzed Synthesis of Trisubstituted Indolizines from 2-Propargyloxypyridines and Methyl Ketones
}

Matthew D. Rossler, Colin T. Hartgerink, Emily E. Zerull, Benjamin L. Boss, Abigail K. Frndak,
Miles M. Mason, Leslie A. Nickerson, Evan O. Romero, Jaimie E. Van de Burg, Richard J. Staples,
Carolyn E. Anderson*

Department of Chemistry and Biochemistry, Calvin College, 1726 Knollcrest Circle SE, Grand Rapids, MI 49546.

\section{Supporting Information}

Representative experimental procedures and tabulated characterization data for all new compounds, details of further optimization studies, CIF file and solid-state packing diagrams for 4, isotope study spectral data, and copies of ${ }^{1} \mathrm{H}$ and ${ }^{13} \mathrm{C}$ NMR spectra for new compounds.

(71 Pages)

General experimental details

S3

Experimental details for newly synthesized compounds

S3-S22

Optimization Studies

S23

Experimental details and solid state packing diagrams for the

S24-S25

X-ray crystal structure of Compound 4

Isotope study spectral data

S26-S27

${ }^{1} \mathrm{H}$ and ${ }^{13} \mathrm{C}$ NMR Spectra of Compound 8a

S28

${ }^{1} \mathrm{H}$ and ${ }^{13} \mathrm{C}$ NMR Spectra of Compound $8 \mathrm{~g}$

S29

${ }^{1} \mathrm{H}$ and ${ }^{13} \mathrm{C}$ NMR Spectra of Compound 10a

$\mathrm{S} 30$

${ }^{1} \mathrm{H}$ and ${ }^{13} \mathrm{C}$ NMR Spectra of Compound 10c

S31

${ }^{1} \mathrm{H}$ and ${ }^{13} \mathrm{C}$ NMR Spectra of Compound 22

$\mathrm{S} 32$

${ }^{1} \mathrm{H}$ and ${ }^{13} \mathrm{C}$ NMR Spectra of Compound 10d

S33

${ }^{1} \mathrm{H}$ and ${ }^{13} \mathrm{C}$ NMR Spectra of Compound 4

${ }^{1} \mathrm{H}$ and ${ }^{13} \mathrm{C}$ NMR Spectra of Compound $7 \mathbf{a}$

S35

${ }^{1} \mathrm{H}$ and ${ }^{13} \mathrm{C}$ NMR Spectra of Compound $7 \mathbf{b}$

S36

${ }^{1} \mathrm{H}$ and ${ }^{13} \mathrm{C}$ NMR Spectra of Compound 7c

S37

${ }^{1} \mathrm{H}$ and ${ }^{13} \mathrm{C}$ NMR Spectra of Compound 7d

S38

${ }^{1} \mathrm{H}$ and ${ }^{13} \mathrm{C}$ NMR Spectra of Compound 7e

S39

${ }^{1} \mathrm{H}$ and ${ }^{13} \mathrm{C}$ NMR Spectra of Compound $7 \mathbf{f}$

S40

${ }^{1} \mathrm{H}$ and ${ }^{13} \mathrm{C}$ NMR Spectra of Compound $7 \mathbf{g}$ 
S2 Supporting Information. Rossler, Hartgerink, Zerull, Anderson et. al.

${ }^{1} \mathrm{H}$ and ${ }^{13} \mathrm{C}$ NMR Spectra of Compound $7 \mathbf{h}$

${ }^{1} \mathrm{H}$ and ${ }^{13} \mathrm{C}$ NMR Spectra of Compound $7 \mathbf{i}$

${ }^{1} \mathrm{H}$ and ${ }^{13} \mathrm{C}$ NMR Spectra of Compound $7 \mathbf{j}$

${ }^{1} \mathrm{H}$ and ${ }^{13} \mathrm{C}$ NMR Spectra of Compound $7 \mathbf{k}$

${ }^{1} \mathrm{H}$ and ${ }^{13} \mathrm{C}$ NMR Spectra of Compound $7 \mathbf{l}$

${ }^{1} \mathrm{H}$ and ${ }^{13} \mathrm{C}$ NMR Spectra of Compound $7 \mathbf{m}$

${ }^{1} \mathrm{H}$ and ${ }^{13} \mathrm{C}$ NMR Spectra of Compound 7n

${ }^{1} \mathrm{H}$ and ${ }^{13} \mathrm{C}$ NMR Spectra of Compound 9a

${ }^{1} \mathrm{H}$ and ${ }^{13} \mathrm{C}$ NMR Spectra of Compound 9aa

${ }^{1} \mathrm{H}$ and ${ }^{13} \mathrm{C}$ NMR Spectra of Compound $\mathbf{9 b}$

${ }^{1} \mathrm{H}$ and ${ }^{13} \mathrm{C}$ NMR Spectra of Compound $\mathbf{9 b b}$

${ }^{1} \mathrm{H}$ and ${ }^{13} \mathrm{C}$ NMR Spectra of Compound 9c

${ }^{1} \mathrm{H}$ and ${ }^{13} \mathrm{C}$ NMR Spectra of Compound 9cc

${ }^{1} \mathrm{H}$ and ${ }^{13} \mathrm{C}$ NMR Spectra of Compound 9cec

${ }^{1} \mathrm{H}$ and ${ }^{13} \mathrm{C}$ NMR Spectra of Compound 9d

${ }^{1} \mathrm{H}$ and ${ }^{13} \mathrm{C}$ NMR Spectra of Compound 9dd

${ }^{1} \mathrm{H}$ and ${ }^{13} \mathrm{C}$ NMR Spectra of Compound 9e

${ }^{1} \mathrm{H}$ and ${ }^{13} \mathrm{C}$ NMR Spectra of Compound $9 \mathbf{f}$

${ }^{1} \mathrm{H}$ and ${ }^{13} \mathrm{C}$ NMR Spectra of Compound 9 ff

${ }^{1} \mathrm{H}$ and ${ }^{13} \mathrm{C}$ NMR Spectra of Compound $9 \mathrm{~g}$

${ }^{1} \mathrm{H}$ and ${ }^{13} \mathrm{C}$ NMR Spectra of Compound 11c

S61

${ }^{1} \mathrm{H}$ and ${ }^{13} \mathrm{C}$ NMR Spectra of Compound 11ce

${ }^{1} \mathrm{H}$ and ${ }^{13} \mathrm{C}$ NMR Spectra of Compound 11cec

${ }^{1} \mathrm{H}$ and ${ }^{13} \mathrm{C}$ NMR Spectra of Compound 11d

${ }^{1} \mathrm{H}$ and ${ }^{13} \mathrm{C}$ NMR Spectra of Compound 11dd

${ }^{1} \mathrm{H}$ and ${ }^{13} \mathrm{C}$ NMR Spectra of Compound 11e

${ }^{1} \mathrm{H}$ and ${ }^{13} \mathrm{C}$ NMR Spectra of Compound 11ee

${ }^{1} \mathrm{H}$ and ${ }^{13} \mathrm{C}$ NMR Spectra of Compound 13

${ }^{1} \mathrm{H}$ and ${ }^{13} \mathrm{C}$ NMR Spectra of Compound 13a

${ }^{1} \mathrm{H}$ and ${ }^{13} \mathrm{C}$ NMR Spectra of Compound 16 
S3 Supporting Information. Rossler, Hartgerink, Zerull, Anderson et. al.

\section{A. Experimental Procedures}

General experimental details. All reagents were purchased from commercial venders. Solvents, including 1-phenylethanol and all acetophenone derivatives, were sparged with argon prior to use. All other reagents were used as received, unless noted otherwise. ${ }^{1} \mathrm{H}$ and ${ }^{13} \mathrm{C}$ NMR spectra were obtained on a $400 \mathrm{MHz}$ NMR spectrometer. Chemical shifts are reported in ppm relative to $\mathrm{CDCl}_{3}$. Multiplicity is indicated as follows: s (singlet); d (doublet); t (triplet); q (quartet); m (multiplet); dd (doublet of doublets); dt (doublet of triplets); app (apparent).

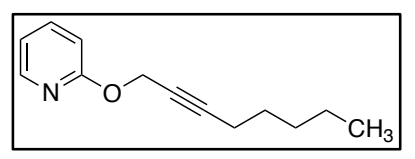

\section{General experimental procedure for the synthesis of 2-}

propargyloxypyridines. 2-(2-octynyloxy)pyridine (1). To 2-chloropyridine $(0.88 \mathrm{~mL}, 8.0 \mathrm{mmol})$ in 1,4-dioxane $(24 \mathrm{~mL})$ was added 2-octyn-1-ol (2.29 $\mathrm{mL}, 16.0 \mathrm{mmol})$. Potassium tert-butoxide (1.35 g, $12.0 \mathrm{mmol})$ was added, and the flask was rinsed with 1,4-dioxane $(12 \mathrm{~mL})$. The reaction was equipped with an air condenser and heated to $98{ }^{\circ} \mathrm{C}$, open to air, for 18 hours. After cooling to room temperature, ethyl acetate $(30 \mathrm{~mL})$ and $\mathrm{H}_{2} \mathrm{O}(30 \mathrm{~mL})$ were added. The layers were separated and the aqueous layer was extracted with ethyl acetate $(30 \mathrm{~mL} \times 2)$. The combined organic layers were washed with 1:1 brine/ $\mathrm{H}_{2} \mathrm{O}(30 \mathrm{~mL})$ and brine $(30 \mathrm{~mL})$ and dried $\left(\mathrm{MgSO}_{4}\right)$. After filtration, the reaction mixture was concentrated in vacuo. Purification by column chromatography $\left(\mathrm{SiO}_{2}, 19: 1\right.$ hexanes/ethyl acetate) provided $1.55 \mathrm{~g}\left(95 \%\right.$ yield) of $\mathbf{1}$ as a yellow oil. ${ }^{1} \mathrm{H}$ NMR (400 MHz, $\left.\mathrm{CDCl}_{3}\right): \delta 8.15(\mathrm{dd}, J=2.0,4.8 \mathrm{~Hz}, 1 \mathrm{H}), 7.56(\mathrm{ddd}, J=2.0,6.8,8.4 \mathrm{~Hz}, 1 \mathrm{H}), 6.88$ (ddd, $J=0.8,4.8,7.2 \mathrm{~Hz}, 1 \mathrm{H}), 6.79(\mathrm{~d}, J=7.6 \mathrm{~Hz}, 1 \mathrm{H}), 4.95(\mathrm{t}, J=2.0 \mathrm{~Hz}, 2 \mathrm{H}), 2.23(\mathrm{tt}, J=2.0,7.2$ $\mathrm{Hz}, 2 \mathrm{H}), 1.52$ (quintet, $J=7.2 \mathrm{~Hz}, 2 \mathrm{H}), 1.24-1.38(\mathrm{~m}, 4 \mathrm{H}), 0.88(\mathrm{t}, J=6.8 \mathrm{~Hz}, 3 \mathrm{H}) ;{ }^{13} \mathrm{C} \mathrm{NMR}(100$ $\left.\mathrm{MHz}, \mathrm{CDCl}_{3}\right): \delta 162.9,147.0,138.8,117.3,111.5,87.4,75.5,54.4,31.2,28.4,22.4,19.1,14.2$; IR (neat): 2932, 2237, 1749, 1594, 1570, 1472, 1432, $1272 \mathrm{~cm}^{-1}$; HRMS (ESI) m/z 226.1201 [226.1208 calcd for $\mathrm{C}_{13} \mathrm{H}_{17} \mathrm{NONa}(\mathrm{M}+\mathrm{Na})^{+}$.

2-(6-Phenyl-2-pentynyloxy)pyridine (8a). Following the general procedure outlined above for the

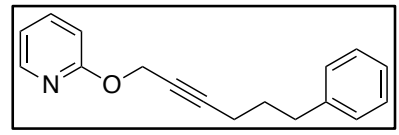
synthesis of compound 1, potassium tert-butoxide (993 $\mathrm{mg}, 8.9 \mathrm{mmol}$ ) was added to 2-chloropyridine $(0.55 \mathrm{~mL}, 5.40 \mathrm{mmol})$ and 6-phenyl-2-pentyn-1-ol ${ }^{1}$ $(1.28 \mathrm{~g}, 7.4 \mathrm{mmol})$ in 1,4-dioxane $(27 \mathrm{~mL})$. After 22 hours, the reaction was worked up and purified by column chromatography ( $\mathrm{SiO}_{2}, 97: 3$ hexanes/ethyl acetate) to afford $1.07 \mathrm{~g}$ (72\% yield) of $\mathbf{8 a}$ as a yellow

(1) Larionov, O. V.; Corey, E. J. J. Am. Chem. Soc. 2008, 130, 2954-2955. 
S4 Supporting Information. Rossler, Hartgerink, Zerull, Anderson et. al.

oil. ${ }^{1} \mathrm{H}$ NMR (400 MHz, $\left.\mathrm{CDCl}_{3}\right): \delta 8.18(\mathrm{~d}, J=2.9 \mathrm{~Hz}, 1 \mathrm{H}), 7.57(\mathrm{t}, J=7.7 \mathrm{~Hz}, 1 \mathrm{H}), 7.25-7.29(\mathrm{~m}, 2 \mathrm{H})$, 7.15-7.20 (m, 3H), $6.88(\mathrm{t}, J=6.0 \mathrm{~Hz}, 1 \mathrm{H}), 6.81(\mathrm{~d}, J=8.4 \mathrm{~Hz}, 1 \mathrm{H}), 4.99(\mathrm{~s}, 2 \mathrm{H}), 2.70(\mathrm{t}, J=7.5 \mathrm{~Hz}$, 2H), $2.25(\mathrm{t}, J=6.9 \mathrm{~Hz}, 2 \mathrm{H}), 1.84$ (quintet, $J=7.4 \mathrm{~Hz}, 2 \mathrm{H}) ;{ }^{13} \mathrm{C} \mathrm{NMR}\left(100 \mathrm{MHz}, \mathrm{CDCl}_{3}\right): \delta 162.7$, 146.8, 141.5, 138.6, 128.5, 128.3, 125.9, 117.2, 111.3, 86.5, 76.0, 54.1, 34.7, 30.0, 18.3; IR (neat): 3024, 2933, 2238, 1495, 1270, $1141 \mathrm{~cm}^{-1}$; HRMS (ESI) m/z 252.1388 [252.1383 calcd for $\left.\mathrm{C}_{17} \mathrm{H}_{18} \mathrm{NO}(\mathrm{M}+\mathrm{H})^{+}\right]$.

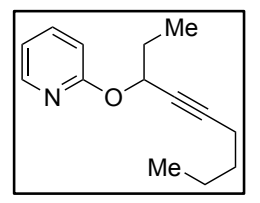

2-(4-Nonynyloxy)pyridine (8g). Following the general procedure outlined above for the synthesis of compound 1, potassium tert-butoxide (329 $\mathrm{mg}, 2.9 \mathrm{mmol}$ ) was added to 2-chloropyridine $(0.22 \mathrm{~mL}, 2.35 \mathrm{mmol})$ and 4-nonyn-3-ol ${ }^{1}$ (412 mg, $\left.2.9 \mathrm{mmol}\right)$ in 1,4-

dioxane $(11 \mathrm{~mL})$. After 22 hours, the reaction was worked up and purified by column chromatography $\left(\mathrm{SiO}_{2}, 19: 1\right.$ hexanes/ethyl acetate) to afford $149 \mathrm{mg}$ (29\% yield) of $\mathbf{8 g}$ as a yellow oil. ${ }^{1} \mathrm{H}$ NMR (400 $\left.\mathrm{MHz}, \mathrm{CDCl}_{3}\right): \delta 8.15(\mathrm{~d}, J=4.4 \mathrm{~Hz}, 1 \mathrm{H}), 7.54(\mathrm{t}, J=7.7 \mathrm{~Hz}, 1 \mathrm{H}), 6.83(\mathrm{t}, J=6.0 \mathrm{~Hz}, 1 \mathrm{H}), 6.74(\mathrm{~d}, J=$ $8.3 \mathrm{~Hz}, 1 \mathrm{H}), 5.59$ (t, $J=5.4 \mathrm{~Hz}, 1 \mathrm{H}), 2.17$ (t, $J=7.0 \mathrm{~Hz}, 2 \mathrm{H}), 1.84-1.90$ (m, 2H), 1.44 (quintet, $J=7.0$ $\mathrm{Hz}, 2 \mathrm{H}), 1.33$ (sextet, $J=7.2 \mathrm{~Hz}, 2 \mathrm{H}), 1.05(\mathrm{t}, J=7.4 \mathrm{~Hz}, 3 \mathrm{H}), 0.84(\mathrm{t}, J=7.2 \mathrm{~Hz}, 3 \mathrm{H}) ;{ }^{13} \mathrm{C} \mathrm{NMR}(100$ $\mathrm{MHz}_{\mathrm{CDCl}}$ ): $\delta 162.8,146.8,138.5,116.8,111.4,85.6,78.6,66.5,30.6,28.6,21.8,18.4,13.5,9.5 ; \mathrm{IR}$ (neat): 2958, 1593, 1468, 1430, $1307 \mathrm{~cm}^{-1}$; HRMS (ESI) m/z 218.1530 [218.1539 calcd for $\mathrm{C}_{14} \mathrm{H}_{20} \mathrm{NO}$ $\left.(\mathrm{M}+\mathrm{H})^{+}\right]$.

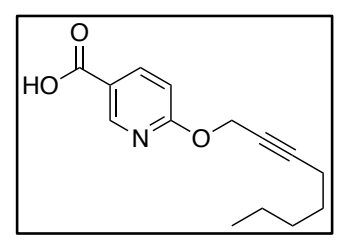

4-Carboxy-2-(2-octynyloxy)pyridine (10a). Following the general procedure outlined above for the synthesis of compound $\mathbf{1}$, potassium tert-butoxide $(2.80 \mathrm{~g}$, $25 \mathrm{mmol})$ was added to 6-chloropyridine carboxylic acid (1.57 g, $10 \mathrm{mmol})$ and 2octyn-1-ol $(2.16 \mathrm{~mL}, 15 \mathrm{mmol})$ in 1,4-dioxane $(46 \mathrm{~mL})$. After 22 hours, ethyl acetate $(30 \mathrm{~mL})$ and $1 \mathrm{M} \mathrm{HCl}(30 \mathrm{~mL})$ were added. The layers were separated and the aqueous layer was extracted with ethyl acetate $(30 \mathrm{~mL}$ x 2$)$. The combined organic layers were washed with $1 \mathrm{M} \mathrm{Hcl}(30$ $\mathrm{mL}), 1: 1$ brine $/ \mathrm{H}_{2} \mathrm{O}(30 \mathrm{~mL})$, and brine $(30 \mathrm{~mL})$ and dried $\left(\mathrm{MgSO}_{4}\right)$. After filtration, the reaction mixture was concentrated in vacuo. Recrystallization from 1:1 ethanol: $\mathrm{H}_{2} \mathrm{O}$ afforded $1.12 \mathrm{~g}$ (45\% yield) of 10a as a white solid. mp: $146-147{ }^{\circ} \mathrm{C},{ }^{1} \mathrm{H}$ NMR (400 MHz, acetone- $\left.d_{6}\right): \delta 8.79(\mathrm{~s}, 1 \mathrm{H}), 8.22(\mathrm{~d}, J=8.6 \mathrm{~Hz}$, $1 \mathrm{H}), 6.90(\mathrm{~d}, J=8.6 \mathrm{~Hz}, 1 \mathrm{H}), 5.03(\mathrm{~s}, 2 \mathrm{H}), 2.21(\mathrm{t}, J=6.6 \mathrm{~Hz}, 2 \mathrm{H}), 1.47$ (q, $J=6.9 \mathrm{~Hz}, 2 \mathrm{H}), 1.38-1.22$ $(\mathrm{m}, 4 \mathrm{H}), 0.85(\mathrm{t}, J=6.7 \mathrm{~Hz}, 3 \mathrm{H}) ;{ }^{13} \mathrm{C} \mathrm{NMR}(100 \mathrm{MHz}$, acetone-d6): $\delta 165.41,165.38,149.6,140.0$, 120.5, 110.7, 86.7, 75.1, 54.3, 30.7, 28.0, 21.9, 18.1, 13.3; IR (neat): 2927, 2538, 2238, 1682, 1603, 1281, $1138 \mathrm{~cm}^{-1}$; HRMS (ESI) m/z 248.1278 [248.1281 calcd for $\left.\mathrm{C}_{14} \mathrm{H}_{18} \mathrm{NO}(\mathrm{M}+\mathrm{H})^{+}\right]$. 
S5 Supporting Information. Rossler, Hartgerink, Zerull, Anderson et. al.

4-(2,2-Dimethylpropanamide)-2-(2-octynyloxy)pyridine (10c). Following the general procedure

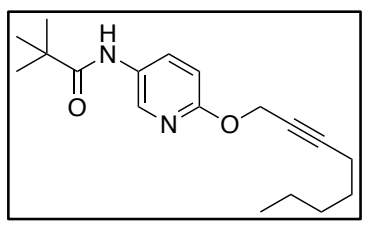
outlined above for the synthesis of compound $\mathbf{1}$, potassium tert-butoxide $(1.25$ g, $11 \mathrm{mmol}$ ) was added to $N$-(6-chloropyridin-3-yl)-2,2-dimethylpropanamide $(1.60 \mathrm{~g}, 7.4 \mathrm{mmol})$ and 2-octyn-1-ol $(1.60 \mathrm{~mL}, 11 \mathrm{mmol})$ in 1,4-dioxane (34 $\mathrm{mL}$ ). After 22 hours, the reaction was worked up and purified by column chromatography ( $\mathrm{SiO}_{2}, 9: 1$ to 3:1 hexanes/ethyl acetate) to afford $1.65 \mathrm{~g}$ (73\% yield) of 10c as a yellow powder. mp: $62-65{ }^{\circ} \mathrm{C},{ }^{1} \mathrm{H}$ NMR (400 MHz, $\mathrm{CDCl}_{3}$ ): $\delta 8.10$ (s, $\left.1 \mathrm{H}\right), 7.90(\mathrm{~d}, J=8.8 \mathrm{~Hz}, 1 \mathrm{H}), 7.37$ (s, 1H), 6.71 (d, $J=8.9 \mathrm{~Hz}, 1 \mathrm{H}), 4.87$ (s, 2H), 2.17 (t, $J=7.0 \mathrm{~Hz}, 2 \mathrm{H}), 1.47$ (t, $J=6.9 \mathrm{~Hz}, 2 \mathrm{H}), 1.26$ (s, 13H), $0.84(\mathrm{t}, J=6.7,3 \mathrm{H}) ;{ }^{13} \mathrm{C} \mathrm{NMR}\left(100 \mathrm{MHz}, \mathrm{CDCl}_{3}\right): \delta 177.1,159.4,138.8,132.8,129.0,110.7$, 87.2, 75.1, 54.4, 39.4, 34.9, 31.0, 28.1, 27.5, 22.1, 18.8, 13.9; IR (neat): 3275, 2928, 2239, 1649, 1483, 1399, 1275, $1254 \mathrm{~cm}^{-1}$; HRMS (ESI) m/z 303.2058 [303.2067 calcd for $\left.\mathrm{C}_{18} \mathrm{H}_{27} \mathrm{~N}_{2} \mathrm{O}_{2}(\mathrm{M}+\mathrm{H})^{+}\right]$.

4-(triisopropylsiloxymethyl)-2-(2-octynyloxy)pyridine (10d). To 6-chloro-3-pyridine methanol ${ }_{22}$ (718mg, $5.0 \mathrm{mmol})$ in dichloromethane $(22 \mathrm{~mL})$ was added imidazole $(408 \mathrm{mg}$, $6.0 \mathrm{mmol})$. Tris(isopropyl)silyl chloride $(1.28 \mathrm{~mL}, 6.0 \mathrm{mmol})$ was added, and the flask was rinsed with dichloromethane $(22 \mathrm{~mL})$. After 16 hours, $\mathrm{H}_{2} \mathrm{O}(30 \mathrm{~mL})$ was added, and the layers were separated. The aqueous layer was then extracted with dichloromethane $(30 \mathrm{~mL} \times 2)$. The combined organic layers were washed with saturated aqueous $\mathrm{NaCO}_{3}(30 \mathrm{~mL})$ and brine $(30 \mathrm{~mL})$ and dried $\left(\mathrm{MgSO}_{4}\right)$. After filtration, the reaction was concentrated in vacuo. Purification by column chromatography $\left(\mathrm{SiO}_{2}, 19: 1\right.$ hexanes/ethyl acetate) provided $1.37 \mathrm{~g}(91 \%$ yield) of TIPS-protected 6-chloro-3-pyridine methanol 22 as a colorless oil. ${ }^{1} \mathrm{H}$ NMR (400 $\left.\mathrm{MHz}, \mathrm{CDCl}_{3}\right)$ : $\delta 8.34(\mathrm{~s}, 1 \mathrm{H}), 7.65(\mathrm{dd}, J=2.0,7.6 \mathrm{~Hz}, 1 \mathrm{H}), 7.28(\mathrm{~d}, J=8.0 \mathrm{~Hz}, 1 \mathrm{H}), 4.81(\mathrm{~s}, 2 \mathrm{H}), 1.10-1.21(\mathrm{~m}, 3 \mathrm{H})$, $1.06(\mathrm{~d}, J=7.2 \mathrm{~Hz}, 18 \mathrm{H}) ;{ }^{13} \mathrm{C} \mathrm{NMR}\left(100 \mathrm{MHz}, \mathrm{CDCl}_{3}\right): \delta 149.9,147.4,136.6,135.9,123.8,72.2,17.9$, 11.9; IR (neat): 2942, 2890, 2865, 1568, 1456, $1098 \mathrm{~cm}^{-1}$.

Following the general procedure outlined above for the synthesis of compound 1, potassium tertbutoxide (589 mg, $11 \mathrm{mmol})$ was added to compound 22 (1.05 g, $3.5 \mathrm{mmol})$ and 2-octyn-1-ol (0.75 mL, $5.3 \mathrm{mmol})$ in 1,4-dioxane $(16 \mathrm{~mL})$. After 22 hours, the reaction was worked up and purified by column chromatography ( $\mathrm{SiO}_{2}, 98: 2$ hexanes/ethyl acetate) to afford $838 \mathrm{mg}$ (61\% yield) of $\mathbf{1 0 d}$ as a clear oil. ${ }^{1} \mathrm{H}$ NMR (400 MHz, $\mathrm{CDCl}_{3}$ ): $\delta 8.06$ (s, 1H), 7.52 (d, $\left.J=8.4 \mathrm{~Hz}, 1 \mathrm{H}\right), 6.71(\mathrm{~d}, J=8.4 \mathrm{~Hz}, 1 \mathrm{H}), 4.89$ (s, 2H), 4.69 (s, 2H), 2.16 (t, $J=6.7 \mathrm{~Hz}, 2 \mathrm{H}), 1.45$ (pentet, $J=6.8 \mathrm{~Hz}, 2 \mathrm{H}), 1.32-1.15$ (m, 4H), 1.15-1.05 
$(\mathrm{m}, 3 \mathrm{H}), 1.02(\mathrm{~d}, J=6.6 \mathrm{~Hz}, 18 \mathrm{H}), 0.81(\mathrm{t}, J=7.6 \mathrm{~Hz}, 6.3 \mathrm{~Hz}, 3 \mathrm{H}) ;{ }^{13} \mathrm{C} \mathrm{NMR}\left(100 \mathrm{MHz}, \mathrm{CDCl}_{3}\right): \delta$ $161.9,144.2,137.1,129.9,110.7,86.8,75.3,62.6,54.1,31.0,28.2,22.1,18.8,17.9,13.9,11.9$; IR (neat): 2939, 2864, 2238, 1486, $1306 \mathrm{~cm}^{-1}$; HRMS (ESI) m/z 390.2815 [390.2823 calcd for $\mathrm{C}_{23} \mathrm{H}_{40} \mathrm{NO}_{2} \mathrm{Si}$ $\left.(\mathrm{M}+\mathrm{H})^{+}\right]$.

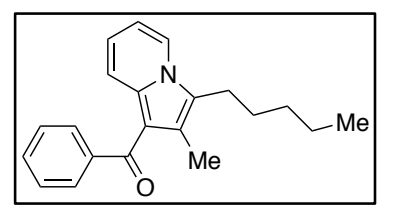

General experimental procedure for the Au(I)-catalyzed formation of trisubstituted indolizines. 1-benzoyl-2-methyl-3-pentylindolizine (4). To 2(2-octynoxy)pyridine $(1,234 \mathrm{mg}, 1.15 \mathrm{mmol})$ in a $\mathrm{G} 10$ microwave vial in an inert atmosphere glovebox was added bis(trifluoromethanesulfonyl)imide (2-dicyclohexylphosphino2',6'-dimethoxy-1,1'-biphenyl)gold(I) (catalyst 5c, $45 \mathrm{mg}, 0.051 \mathrm{mmol}), 1$-phenylethanol (0.60 mL) and acetophenone $(3.9 \mathrm{~mL})$. After closing the vial and removing it from the glovebox, the vial was heated at $100{ }^{\circ} \mathrm{C}$ for 18 hours. After cooling to room temperature, the reaction was filtered through a cotton plug, rinsing with ethyl acetate. The volatiles were removed in vacuo followed by removal of the remainder of the acetophenone by Kugelrohr distillation. The residual was purified by column chromatography (19:1 to 9:1 hexanes/ethyl acetate) to afford $190 \mathrm{mg}$ (54\% yield) of $\mathbf{4}$ as a yellow solid. mp: $91-92{ }^{\circ} \mathrm{C}$; ${ }^{1} \mathrm{H}$ NMR (400 MHz, $\left.C D C l_{3}\right): \delta 7.83(\mathrm{~d}, J=4.0 \mathrm{~Hz}, 1 \mathrm{H}), 7.66(\mathrm{~d}, J=7.8 \mathrm{~Hz}, 2 \mathrm{H}), 7.47(\mathrm{t}, J=6.6 \mathrm{~Hz}$, 1H), $7.41(\mathrm{t}, J=7.8 \mathrm{~Hz}, 2 \mathrm{H}), 6.83(\mathrm{t}, J=7.8 \mathrm{~Hz}, 1 \mathrm{H}), 6.68(\mathrm{t}, J=6.4 \mathrm{~Hz}, 1 \mathrm{H}), 2.83(\mathrm{t}, J=7.8 \mathrm{~Hz}, 2 \mathrm{H})$, $2.23(\mathrm{~s}, 3 \mathrm{H}), 1.57$ (pentet, $J=8.0 \mathrm{~Hz}, 2 \mathrm{H}), 1.28-1.37(\mathrm{~m}, 4 \mathrm{H}), 0.87(\mathrm{t}, J=6.4 \mathrm{~Hz}, 3 \mathrm{H}) ;{ }^{13} \mathrm{C}$ NMR $(100$ $\left.\mathrm{MHz}_{\mathrm{CDCl}}\right): \delta 192.1,142.3,136.1,130.8,128.7,128.2,124.5,124.3,122.3,121.1,119.4,112.3,112.2$, 31.6, 26.9, 23.4, 22.5, 14.0, 11.8; IR (neat): 3058, 2927, 2859, 1612, 1497, 1393, $1241 \mathrm{~cm}^{-1}$; HRMS (ESI) $\mathrm{m} / \mathrm{z} 306.1842\left[306.1852\right.$ calcd for $\left.\mathrm{C}_{21} \mathrm{H}_{24} \mathrm{NO}(\mathrm{M}+\mathrm{H})^{+}\right]$.

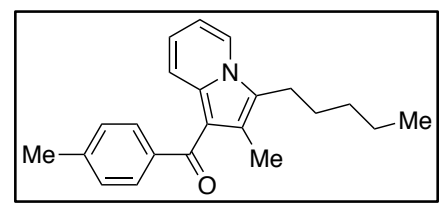

1-(4-Methylbenzoyl)-2-methyl-3-pentylindolizine (7a). Following the general procedure outlined above for the synthesis of compound $\mathbf{4}$, catalyst 5c (24 mg, $0.027 \mathrm{mmol})$, 1-phenylethanol $(0.30 \mathrm{~mL})$ and 4'methylacetophenone $(2.0 \mathrm{~mL})$ were added to 2-(2-octynoxy)pyridine (1, $103 \mathrm{mg}, 0.51 \mathrm{mmol})$. After 18 hours, the reaction was worked up and purified by column chromatography $\left(\mathrm{SiO}_{2}, 19: 1\right.$ hexanes/ethyl acetate) to afford $64 \mathrm{mg}\left(40 \%\right.$ yield) of $7 \mathbf{a}$ as a yellow solid. mp: $81-83{ }^{\circ} \mathrm{C} ;{ }^{1} \mathrm{H} \mathrm{NMR}\left(400 \mathrm{MHz}, C D C l_{3}\right)$ : $\delta 7.82(\mathrm{~d}, J=6.8 \mathrm{~Hz}, 1 \mathrm{H}), 7.59(\mathrm{~d}, J=8.0 \mathrm{~Hz}, 2 \mathrm{H}), 7.42(\mathrm{~d}, J=8.8 \mathrm{~Hz}, 1 \mathrm{H}), 7.22(\mathrm{~d}, J=8.0 \mathrm{~Hz}, 2 \mathrm{H})$, $6.83(\mathrm{ddd}, J=0.8,6.6,9.0 \mathrm{~Hz}, 1 \mathrm{H}), 6.67(\mathrm{td}, J=1.2,6.8 \mathrm{~Hz}, 1 \mathrm{H}), 2.84(\mathrm{t}, J=7.6 \mathrm{~Hz}, 2 \mathrm{H}), 2.41(\mathrm{~s}, 3 \mathrm{H})$, $2.25(\mathrm{~s}, 3 \mathrm{H}), 1.53-1.63(\mathrm{~m}, 2 \mathrm{H}), 1.30-1.38(\mathrm{~m}, 4 \mathrm{H}), 0.89(\mathrm{t}, J=7.0 \mathrm{~Hz}, 3 \mathrm{H}) ;{ }^{13} \mathrm{C} \mathrm{NMR}(100 \mathrm{MHz}$, $\left.\mathrm{CDCl}_{3}\right): \delta 192.0,141.3,139.4,135.8,129.1,128.8,124.3,124.2,122.3,120.8,119.4,112.5,112.0,31.6$, 
S7 Supporting Information. Rossler, Hartgerink, Zerull, Anderson et. al.

26.9, 23.4, 22.5, 21.6, 14.0, 11.8; IR (neat): 3024, 2922, 2856, 1600, 1492, $1389 \mathrm{~cm}^{-1}$; HRMS (ESI) m/z 320.2009 [320.2011 calcd for $\left.\mathrm{C}_{22} \mathrm{H}_{26} \mathrm{NO}(\mathrm{M}+\mathrm{H})^{+}\right]$.

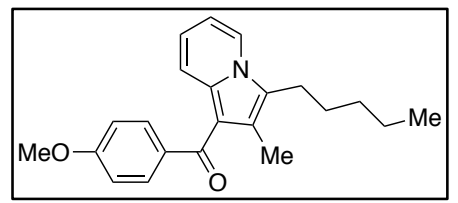

1-(4-Methyloxybenzoyl)-2-methyl-3-pentylindolizine (7b). Following the general procedure outlined above for the synthesis of compound $\mathbf{4}$, catalyst 5c (25 mg, $0.028 \mathrm{mmol})$, 1-phenylethanol $(0.30 \mathrm{~mL})$ and 4'methoxyacetophenone $(2.56 \mathrm{~g}, 17.0 \mathrm{mmol})$ were added to 2-(2-octynoxy)pyridine $(1,61 \mathrm{mg}, 0.30$ mmol). After 18 hours, the reaction was worked up and purified by column chromatography $\left(\mathrm{SiO}_{2}, 97: 3\right.$ to $19: 1$ to $9: 1$ hexanes/ethyl acetate) to afford $66 \mathrm{mg}$ (40\% yield) of $7 \mathbf{b}$ as a thick yellow oil. ${ }^{1} \mathrm{H}$ NMR (400 MHz, $\left.C D C l_{3}\right): \delta 7.82(\mathrm{~d}, J=6.8 \mathrm{~Hz}, 1 \mathrm{H}), 7.70(\mathrm{dt}, J=2.4,9.4 \mathrm{~Hz}, 2 \mathrm{H}), 7.42(\mathrm{~d}, J=8.8 \mathrm{~Hz}, 1 \mathrm{H})$, $6.92(\mathrm{dt}, J=2.2,9.4 \mathrm{~Hz}, 2 \mathrm{H}), 6.82(\mathrm{ddd}, J=1.0,6.8,9.0 \mathrm{~Hz}, 1 \mathrm{H}), 6.66(\mathrm{td}, J=1.2,6.8 \mathrm{~Hz}, 1 \mathrm{H}), 3.86$ (s, $3 \mathrm{H}), 2.85(\mathrm{t}, J=7.6 \mathrm{~Hz}, 2 \mathrm{H}), 2.27(\mathrm{~s}, 3 \mathrm{H}), 1.52-1.63(\mathrm{~m}, 2 \mathrm{H}), 1.30-1.39(\mathrm{~m}, 4 \mathrm{H}), 0.89(\mathrm{t}, J=7.0 \mathrm{~Hz}$, $3 \mathrm{H}) ;{ }^{13} \mathrm{C} \mathrm{NMR}\left(100 \mathrm{MHz}, \mathrm{CDCl}_{3}\right): \delta 191.1,162.1,135.5,134.6,131.3,124.2,124.0,122.2,120.5,119.3$, 113.3, 112.5, 111.9, 55.4, 31.6, 27.0, 23.5, 22.5, 14.0, 11.7; IR (neat): 3069, 2924, 2855, 1596, 1494, 1389, 1241, $1029 \mathrm{~cm}^{-1}$; HRMS (ESI) m/z 336.1951 [336.1958 calcd for $\mathrm{C}_{22} \mathrm{H}_{26} \mathrm{NO}_{2}(\mathrm{M}+\mathrm{H})^{+}$].

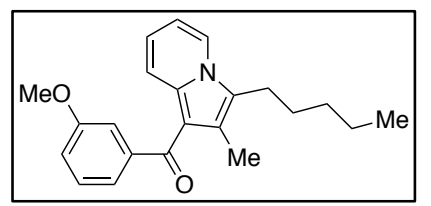

1-(3-Methyloxybenzoyl)-2-methyl-3-pentylindolizine (7c). Following the general procedure outlined above for the synthesis of compound 4 , catalyst $\mathbf{5 c}$ (23 mg, $0.026 \mathrm{mmol})$, 1-phenylethanol $(0.30 \mathrm{~mL})$ and 3'methoxyacetophenone $(2.0 \mathrm{~mL})$ were added to 2-(2-octynoxy)pyridine (1, $120 \mathrm{mg}, 0.59 \mathrm{mmol})$. After 18 hours, the reaction was worked up and purified by column chromatography $\left(\mathrm{SiO}_{2}, 97: 3\right.$ hexanes/ethyl acetate) to afford $75 \mathrm{mg}$ (38\% yield) of $7 \mathrm{c}$ as a thick yellow oil. ${ }^{1} \mathrm{H}$ NMR (400 MHz, $\left.C D C l_{3}\right): \delta 7.83$ (d, $J=6.8 \mathrm{~Hz}, 1 \mathrm{H}), 7.43(\mathrm{~d}, J=9.2 \mathrm{~Hz}, 1 \mathrm{H}), 7.32$ (t, $J=8.0 \mathrm{~Hz}, 1 \mathrm{H}), 7.23$ (dd, $J=1.6,8.4 \mathrm{~Hz}, 2 \mathrm{H}), 7.02$ $7.06(\mathrm{~m}, 1 \mathrm{H}), 6.85(\mathrm{ddd}, J=1.2,6.8,9.0 \mathrm{~Hz}, 1 \mathrm{H}), 6.69(\mathrm{td}, J=1.2,6.8 \mathrm{~Hz}, 1 \mathrm{H}), 3.82(\mathrm{~s}, 3 \mathrm{H}), 2.84(\mathrm{t}, J$ $=7.6 \mathrm{~Hz}, 2 \mathrm{H}), 2.25(\mathrm{~s}, 3 \mathrm{H}), 1.52-1.63(\mathrm{~m}, 2 \mathrm{H}), 1.29-1.39(\mathrm{~m}, 4 \mathrm{H}), 0.89(\mathrm{t}, J=7.0 \mathrm{~Hz}, 3 \mathrm{H}) ;{ }^{13} \mathrm{C} \mathrm{NMR}$ (100 MHz, $\left.\mathrm{CDCl}_{3}\right): \delta 191.7,159.5,143.7,136.1,129.2,124.5,124.3,122.4,121.3,121.2,119.4,117.2$, 113.0, 112.3, 112.2, 55.4, 31.6, 26.9, 23.4, 22.5, 14.0, 11.8; IR (neat): 3066, 2925, 2856, 1519, 1493, $1252,1043 \mathrm{~cm}^{-1}$; HRMS (ESI) m/z 336.1974 [336.1958 calcd for $\mathrm{C}_{22} \mathrm{H}_{26} \mathrm{NO}_{2}(\mathrm{M}+\mathrm{H})^{+}$]. 
Supporting Information. Rossler, Hartgerink, Zerull, Anderson et. al.

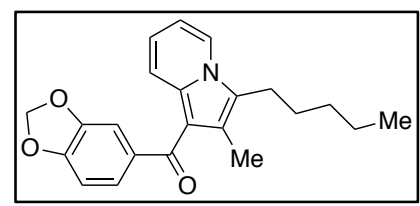

Following the general procedure outlined above for the synthesis of compound 4, catalyst 5c (23 mg, $0.026 \mathrm{mmol})$, 1-phenylethanol $(0.30 \mathrm{~mL})$ and 2H-1,3-benzodioxole methyl ketone (2.80 g, 17.0) were added to 2-(2-octynoxy)pyridine (1, 100 $\mathrm{mg}, 0.49 \mathrm{mmol}$ ). After 18 hours, the reaction was worked up and purified by column chromatography $\left(\mathrm{SiO}_{2}, 19: 1\right.$ to 9:1 hexanes/ethyl acetate) to afford $65 \mathrm{mg}\left(38 \%\right.$ yield) of $7 \mathbf{d}$ as a thick yellow oil. ${ }^{1} \mathrm{H}$ NMR (400 MHz, $C D C l$ ): $\delta 7.82(\mathrm{~d}, J=6.8 \mathrm{~Hz}, 1 \mathrm{H}), 7.46(\mathrm{~d}, J=9.0 \mathrm{~Hz}, 1 \mathrm{H}), 7.20-7.29(\mathrm{~m}, 2 \mathrm{H}), 6.78-$ $6.89(\mathrm{~m}, 2 \mathrm{H}), 6.67(\mathrm{t}, J=6.7 \mathrm{~Hz}, 1 \mathrm{H}), 6.03(\mathrm{~s}, 2 \mathrm{H}), 2.84(\mathrm{t}, J=7.5 \mathrm{~Hz}, 2 \mathrm{H}), 2.26(\mathrm{~s}, 3 \mathrm{H}), 1.52-1.64(\mathrm{~m}$, 2H), 1.28-1.40 (m, 4H), 0.89 (app t, $\left.J=6.0 \mathrm{~Hz}, 3 \mathrm{H}) ;{ }^{13} \mathrm{C} \mathrm{NMR} \mathrm{(100} \mathrm{MHz,} \mathrm{CDCl}_{3}\right): \delta 190.6,150.2$, 147.6, 136.3, 135.6, 124.8, 124.3, 124.0, 122.3, 120.7, 119.3, 112.3, 112.0, 109.3, 107.7, 101.5, 31.6, 26.9, 23.4, 22.5, 14.0, 11.7; IR (neat): 2925, 2857, 1594, 1485, 1437, $1246 \mathrm{~cm}^{-1}$; HRMS (ESI) m/z $350.1730\left[350.1751\right.$ calcd for $\left.\mathrm{C}_{22} \mathrm{H}_{24} \mathrm{NO}_{3}(\mathrm{M}+\mathrm{H})^{+}\right]$.

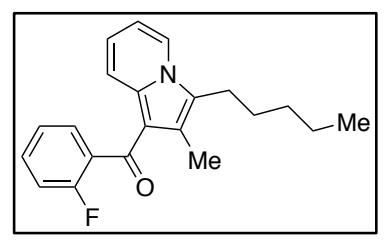

1-(2-Fluorobenzoyl)-2-methyl-3-pentylindolizine (7e). Following the general procedure outlined above for the synthesis of compound 4, catalyst 5c (23 mg, $0.026 \mathrm{mmol})$, 1-butanol $(0.23 \mathrm{~mL})$ and 2'-fluoroacetophenone $(2.0 \mathrm{~mL})$ were added to 2-(2-octynoxy)pyridine (1, $103 \mathrm{mg}, 0.51 \mathrm{mmol})$. After 18 hours, the reaction was worked up and purified by column chromatography $\left(\mathrm{SiO}_{2}, 19: 1\right.$ to 9:1 hexanes/ethyl acetate) to afford $68 \mathrm{mg}\left(41 \%\right.$ yield) of $7 \mathrm{e}$ as a thick yellow oil. ${ }^{1} \mathrm{H}$ NMR $\left(400 \mathrm{MHz}, C D C l_{3}\right): \delta 7.86$ $(\mathrm{d}, J=6.7 \mathrm{~Hz}, 1 \mathrm{H}), 7.66(\mathrm{~d}, J=9.1 \mathrm{~Hz}, 1 \mathrm{H}), 7.39-7.47(\mathrm{~m}, 2 \mathrm{H}), 7.18-7.26(\mathrm{~m}, 1 \mathrm{H}), 7.12(\mathrm{t}, J=8.8 \mathrm{~Hz}$, 1H), $6.97(\mathrm{t}, J=7.8 \mathrm{~Hz}, 1 \mathrm{H}), 6.76(\mathrm{t}, J=6.8 \mathrm{~Hz}, 1 \mathrm{H}), 2.82$ (t, $J=7.5 \mathrm{~Hz}, 2 \mathrm{H}), 2.15(\mathrm{~s}, 3 \mathrm{H}), 1.50-1.60$ $(\mathrm{m}, 2 \mathrm{H}), 1.27-1.38(\mathrm{~m}, 4 \mathrm{H}), 0.88(\mathrm{t}, J=5.9 \mathrm{~Hz}, 3 \mathrm{H}) ;{ }^{13} \mathrm{C} \mathrm{NMR}\left(100 \mathrm{MHz}, \mathrm{CDCl}_{3}\right): \delta 186.7,159.2(\mathrm{~d}, J$ $=247 \mathrm{~Hz}), 136.8,131.6(\mathrm{~d}, J=17 \mathrm{~Hz}), 131.2(\mathrm{~d}, J=7.9 \mathrm{~Hz}), 129.4(\mathrm{~d}, J=3.7 \mathrm{~Hz}), 124.8,124.4(\mathrm{~d}, J$ $=3.4 \mathrm{~Hz}), 124.3,122.9,122.7,119.3,116.0(\mathrm{~d}, J=21.7 \mathrm{~Hz}), 113.0,112.6,31.5,26.9,23.3,22.5$, 14.0, 11.4; IR (neat): 3059, 2926, 2857, 1602, 1490, $1393 \mathrm{~cm}^{-1}$; HRMS (ESI) m/z 324.1747 [324.1758 calcd for $\left.\mathrm{C}_{21} \mathrm{H}_{23} \mathrm{FNO}(\mathrm{M}+\mathrm{H})^{+}\right]$.

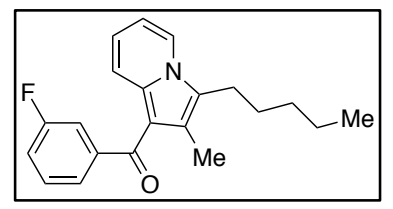

1-(3-Fluorobenzoyl)-2-methyl-3-pentylindolizine (7f). Following the general procedure outlined above for the synthesis of compound 4, catalyst 5c (22 mg, $0.026 \mathrm{mmol}), 1$-phenylethanol $(0.30 \mathrm{~mL})$ and 3'-fluoroacetophenone $(2.0 \mathrm{~mL})$ were added to 2-(2-octynoxy)pyridine (1, $103 \mathrm{mg}, 0.51 \mathrm{mmol})$. After 18 hours, the reaction was worked up and purified by column chromatography $\left(\mathrm{SiO}_{2}, 97: 3\right.$ to 19:1 hexanes/ethyl acetate) to afford $109 \mathrm{mg}$ 
(66\% yield) of $7 \mathbf{f}$ as a thick yellow oil. ${ }^{1} \mathrm{H}$ NMR (400 MHz, $\left.C D C l_{3}\right): \delta 7.85(\mathrm{~d}, J=6.8 \mathrm{~Hz}, 1 \mathrm{H}), 7.32-$ $7.49(\mathrm{~m}, 4 \mathrm{H}), 7.19(\mathrm{t}, J=8.2 \mathrm{~Hz}, 1 \mathrm{H}), 6.90$ (t, $J=7.9 \mathrm{~Hz}, 1 \mathrm{H}), 6.73(\mathrm{t}, J=6.8 \mathrm{~Hz}, 1 \mathrm{H}), 2.84(\mathrm{t}, J=7.5$ $\mathrm{Hz}, 2 \mathrm{H}), 2.22(\mathrm{~s}, 3 \mathrm{H}), 1.53-1.64(\mathrm{~m}, 2 \mathrm{H}), 1.29-1.40(\mathrm{~m}, 4 \mathrm{H}), 0.89(\mathrm{t}, J=6.4 \mathrm{~Hz}, 3 \mathrm{H}) ;{ }^{13} \mathrm{C} \mathrm{NMR}(100$ $\left.\mathrm{MHz}, \mathrm{CDCl}_{3}\right): \delta 190.3(\mathrm{~d}, J=1.9 \mathrm{~Hz}), 162.6(\mathrm{~d}, J=246 \mathrm{~Hz}), 144.5(\mathrm{~d}, J=6.1 \mathrm{~Hz}), 136.3,129.9(\mathrm{~d}, J=$ $7.7 \mathrm{~Hz}), 124.8,124.4(\mathrm{~d}, J=3.0 \mathrm{~Hz}), 124.2,122.5,121.7,119.3,117.6(\mathrm{~d}, J=21 \mathrm{~Hz}), 115.5(\mathrm{~d}, J=22$ Hz), 112.5, 11.8, 31.5, 26.9, 23.4, 22.5, 14.0, 11.8; IR (neat): 3067, 2926, 2857, 1580, 1492, 1391, 1250 $\mathrm{cm}^{-1}$; HRMS (ESI) m/z 324.1739 [324.1758 calcd for $\left.\mathrm{C}_{21} \mathrm{H}_{23} \mathrm{FNO}(\mathrm{M}+\mathrm{H})^{+}\right]$.

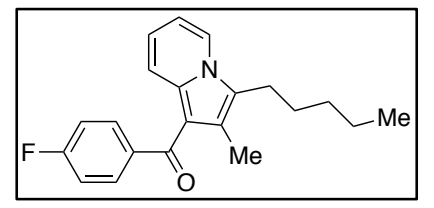

1-(4-Fluorobenzoyl)-2-methyl-3-pentylindolizine (7g). Following the general procedure outlined above for the synthesis of compound 4, catalyst 5c $(23 \mathrm{mg}, 0.026 \mathrm{mmol})$, 1-phenylethanol $(0.30 \mathrm{~mL})$ and 4'fluoroacetophenone $(2.0 \mathrm{~mL})$ were added to 2-(2-octynoxy)pyridine (1, $110 \mathrm{mg}, 0.54 \mathrm{mmol})$. After 18 hours, the reaction was worked up and purified by column chromatography $\left(\mathrm{SiO}_{2}, 97: 3\right.$ to $19: 1$ hexanes/ethyl acetate) to afford $89 \mathrm{mg}$ (51\% yield) of $7 \mathrm{~g}$ as a yellow solid. mp: $66-68{ }^{\circ} \mathrm{C} ;{ }^{1} \mathrm{H} \mathrm{NMR}(400$ $\left.\mathrm{MHz}, C D C l_{3}\right): \delta 7.84(\mathrm{~d}, J=6.8 \mathrm{~Hz}, 1 \mathrm{H}), 7.70(\mathrm{t}, J=6.9 \mathrm{~Hz}, 2 \mathrm{H}), 7.42(\mathrm{~d}, J=9.0 \mathrm{~Hz}, 1 \mathrm{H}), 7.10(\mathrm{t}, J=$ $8.4 \mathrm{~Hz}, 2 \mathrm{H}), 6.87(\mathrm{t}, J=7.9 \mathrm{~Hz}, 1 \mathrm{H}), 6.70(\mathrm{t}, J=6.8 \mathrm{~Hz}, 1 \mathrm{H}), 2.85(\mathrm{t}, J=7.5 \mathrm{~Hz}, 2 \mathrm{H}), 2.23(\mathrm{~s}, 3 \mathrm{H}), 1.52-$ $1.63(\mathrm{~m}, 2 \mathrm{H}), 1.29-1.40(\mathrm{~m}, 4 \mathrm{H}), 0.89(\operatorname{app~t}, J=6.1 \mathrm{~Hz}, 3 \mathrm{H}) ;{ }^{13} \mathrm{C} \mathrm{NMR}\left(100 \mathrm{MHz}, \mathrm{CDCl}_{3}\right): \delta 190.6$, $164.5(\mathrm{~d}, J=250 \mathrm{~Hz}), 138.4(\mathrm{~d}, J=2.8 \mathrm{~Hz}), 136.0,131.3(\mathrm{~d}, J=8.7 \mathrm{~Hz}), 124.6,124.1,122.4,121.3$, 119.2, $115.2(\mathrm{~d}, J=21.5 \mathrm{~Hz}), 112.3,112.1,31.6,26.9,23.4,22.5,14.0,11.8$; IR (neat): 3066, 2926, 2857, 1597, 1492, 1390, $1235 \mathrm{~cm}^{-1}$; HRMS (ESI) m/z 324.1727 [324.1758 calcd for $\mathrm{C}_{21} \mathrm{H}_{23} \mathrm{FNO}$ $\left.(\mathrm{M}+\mathrm{H})^{+}\right]$.

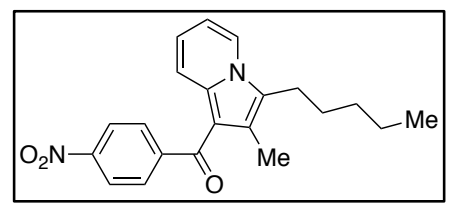

1-(4-Nitrobenzoyl)-2-methyl-3-pentylindolizine (7h). Following the general procedure outlined above for the synthesis of compound 4 , catalyst 5c (23 mg, $0.026 \mathrm{mmol}), 1$-butanol (0.3 mL) and 4'-nitroacetophenone (1.41 $\mathrm{g}, 8.5 \mathrm{mmol})$ were added to 2-(2-octynoxy)pyridine $(1,116 \mathrm{mg}, 0.57 \mathrm{mmol})$. After 18 hours, the reaction was worked up and purified by column chromatography $\left(\mathrm{SiO}_{2}, 19: 1\right.$ hexanes/ethyl acetate) to afford 147 mg (73\% yield) of $7 \mathbf{h}$ as a bright red solid. mp: $93-95{ }^{\circ} \mathrm{C} ;{ }^{1} \mathrm{H}$ NMR $\left(400 \mathrm{MHz}, C D C l_{3}\right): \delta 8.29(\mathrm{dt}, J=$ 2.4, $8.8 \mathrm{~Hz}, 2 \mathrm{H}), 7.89(\mathrm{~d}, J=6.8 \mathrm{~Hz}, 1 \mathrm{H}), 7.78$ (dt, $J=2.4,8.8 \mathrm{~Hz}, 2 \mathrm{H}), 7.49$ (dt, $J=1.2,8.8 \mathrm{~Hz}, 1 \mathrm{H})$, $6.97(\mathrm{ddd}, J=1.0,6.8,9.0 \mathrm{~Hz}, 1 \mathrm{H}), 6.78(\mathrm{dt}, J=1.2,6.8 \mathrm{~Hz}, 1 \mathrm{H}), 2.84(\mathrm{t}, J=7.6 \mathrm{~Hz}, 2 \mathrm{H}), 2.16(\mathrm{~s}, 3 \mathrm{H})$, 1.51-1.63 (m, 2H), 1.36 (heptet, $J=6.0 \mathrm{~Hz}, 4 \mathrm{H}), 0.89$ (t, $J=7.0 \mathrm{~Hz}, 3 \mathrm{H}) ;{ }^{13} \mathrm{C} \mathrm{NMR}\left(100 \mathrm{MHz}, \mathrm{CDCl}_{3}\right)$ : $\delta 189.3,148.8,148.2,136.7,129.3,125.3,124.1,123.6,122.8,122.7,119.2,113.0,111.5,31.5,26.9$, 
S10 Supporting Information. Rossler, Hartgerink, Zerull, Anderson et. al.

23.4, 22.5, 14.0, 12.1; IR (neat): 3104, 2926, 2857, 1519, 1491, $1344 \mathrm{~cm}^{-1}$; HRMS (ESI) m/z 351.1697 [351.1703 calcd for $\left.\mathrm{C}_{21} \mathrm{H}_{23} \mathrm{~N}_{2} \mathrm{O}_{3}(\mathrm{M}+\mathrm{H})^{+}\right]$.

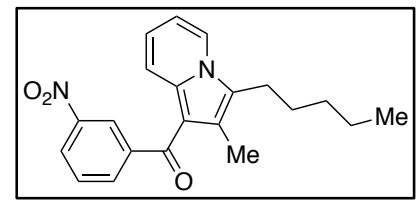

1-(3-Nitrobenzoyl)-2-methyl-3-pentylindolizine (7i). Following the general procedure outlined above for the synthesis of compound 4, catalyst $5 \mathrm{c}$ ( $22 \mathrm{mg}$, $0.026 \mathrm{mmol}), 1$-phenylethanol $(0.3 \mathrm{~mL})$ and 3'-nitroacetophenone $(2.80 \mathrm{~g}$, $17.0 \mathrm{mmol}$ ) were added to 2-(2-octynoxy)pyridine (1, $116 \mathrm{mg}, 0.57 \mathrm{mmol})$. After 18 hours, the reaction was worked up and purified by column chromatography $\left(\mathrm{SiO}_{2}, 9: 1\right.$ to $85: 15$ to 3:1 hexanes/ethyl acetate) to afford $152 \mathrm{mg}\left(76 \%\right.$ yield) of $7 \mathbf{i}$ as a thick amber oil. ${ }^{1} \mathrm{H}$ NMR $\left(400 \mathrm{MHz}, C D C l_{3}\right): \delta 8.48(\mathrm{~s}, 1 \mathrm{H})$, $8.35(\mathrm{~d}, J=8.2 \mathrm{~Hz}, 1 \mathrm{H}), 8.01(\mathrm{~d}, J=6.8 \mathrm{~Hz}, 1 \mathrm{H}), 7.89$ (d, $J=6.8 \mathrm{~Hz}, 1 \mathrm{H}), 7.64(\mathrm{t}, J=7.8 \mathrm{~Hz}, 1 \mathrm{H}), 7.53$ $(\mathrm{d}, J=9.0 \mathrm{~Hz}, 1 \mathrm{H}), 6.96(\mathrm{t}, J=7.8 \mathrm{~Hz}, 1 \mathrm{H}), 6.78(\mathrm{t}, J=6.8 \mathrm{~Hz}, 1 \mathrm{H}), 2.85(\mathrm{t}, J=7.5 \mathrm{~Hz}, 2 \mathrm{H}), 2.217(\mathrm{~s}$, $3 \mathrm{H}), 1.53-1.65(\mathrm{~m}, 2 \mathrm{H}), 1.29-1.41(\mathrm{~m}, 4 \mathrm{H}), 0.89(\mathrm{t}, J=6.4 \mathrm{~Hz}, 3 \mathrm{H}) ;{ }^{13} \mathrm{C} \mathrm{NMR}\left(100 \mathrm{MHz}, \mathrm{CDCl}_{3}\right): \delta$ $188.7,148.0,143.8,136.7,134.5,129.5,125.22,125.18,123.9,123.7,122.8,122.6,119.1,113.0,11.4$, 31.5, 26.9, 23.4, 22.5, 14.0, 12.1; IR (neat): 3082, 2925, 2857, 1604, 1528, 1491, 1391, $1345 \mathrm{~cm}^{-1}$; HRMS (ESI) m/z 351.1712 [351.1703 calcd for $\mathrm{C}_{21} \mathrm{H}_{23} \mathrm{~N}_{2} \mathrm{O}_{3}(\mathrm{M}+\mathrm{H})^{+}$].

1-(3,5-Ditrifluoromethylbenzoyl)-2-methyl-3-pentylindolizine (7j). Following the general procedure

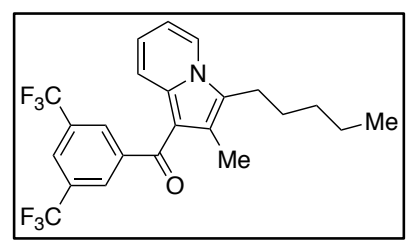

outlined above for the synthesis of compound 4, catalyst 5c (44 mg, 0.050 mmol), 1-phenylethanol $(0.6 \mathrm{~mL})$ and 3',5'-ditrifluoromethylacetophenone (4.0 mL) were added to 2-(2-octynoxy)pyridine (1, $204 \mathrm{mg}, 1.0 \mathrm{mmol})$. After 18 hours, the reaction was worked up and purified by column chromatography $\left(\mathrm{SiO}_{2}, 97: 3\right.$ hexanes/ethyl acetate) to afford $353 \mathrm{mg}$ (80\% yield) of 7j as a thick yellow oil. ${ }^{1} \mathrm{H}$ NMR (400 MHz, $C D C l$ ): $\delta 8.12(\mathrm{~s}, 2 \mathrm{H}), 8.00(\mathrm{~s}, 1 \mathrm{H}), 7.91(\mathrm{~d}, J=6.9 \mathrm{~Hz}, 1 \mathrm{H}), 7.61(\mathrm{~d}$, $J=9.0 \mathrm{~Hz}, 1 \mathrm{H}), 7.02(\mathrm{t}, J=7.7 \mathrm{~Hz}, 1 \mathrm{H}), 6.81(\mathrm{t}, J=6.7 \mathrm{~Hz}, 1 \mathrm{H}), 2.86(\mathrm{t}, J=7.5 \mathrm{~Hz}, 2 \mathrm{H}), 2.12(\mathrm{~s}, 3 \mathrm{H})$, 1.53-1.66 (m, 2H), 1.29-1.41 (m, 4H), $0.89(\mathrm{t}, J=6.5 \mathrm{~Hz}, 3 \mathrm{H}) ;{ }^{13} \mathrm{C}$ NMR $\left(100 \mathrm{MHz}, \mathrm{CDCl}_{3}\right): \delta 187.8$, 144.0, 136.8, $131.6(\mathrm{q}, J=34 \mathrm{~Hz}), 129.0(\mathrm{q}, J=3.5 \mathrm{~Hz}), 125.4,123.9$ (pentet, $J=4.0 \mathrm{~Hz}), 123.7$, $123.1(\mathrm{q}, J=271 \mathrm{~Hz}), 123.0,122.9,119.0,113.2,111.1,31.5,26.9,23.4,22.5,13.9,12.1$; IR (neat): 2928, 2860, 1626, 1493, 1368, 1275, $1129 \mathrm{~cm}^{-1}$; HRMS (ESI) m/z 442.1625 [442.1600 calcd for $\left.\mathrm{C}_{23} \mathrm{H}_{22} \mathrm{~F}_{6} \mathrm{NO}(\mathrm{M}+\mathrm{H})^{+}\right]$. 
S11 Supporting Information. Rossler, Hartgerink, Zerull, Anderson et. al.

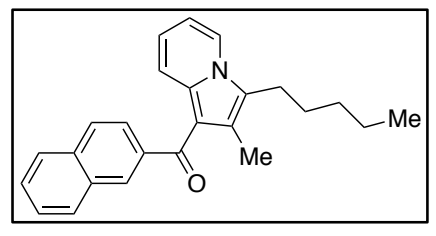

1-naphthoyl-2-methyl-3-pentylindolizine (7k). Following the general procedure outlined above for the synthesis of compound 4 , catalyst 5 c (23 $\mathrm{mg}, 0.026 \mathrm{mmol}), 1-$ phenylethanol $(0.3 \mathrm{~mL})$ and naphthyl methyl ketone (2.95 g, $17.0 \mathrm{mmol})$ were added to 2-(2-octynoxy)pyridine (1, $104 \mathrm{mg}, 0.51$ $\mathrm{mmol})$. After 18 hours, the reaction was worked up and purified by column chromatography $\left(\mathrm{SiO}_{2}, 97: 3\right.$ to $19: 1$ to $9: 1$ hexanes/ethyl acetate) to afford $113 \mathrm{mg}$ (62\% yield) of $7 \mathbf{k}$ as a thick yellow oil. ${ }^{1} \mathrm{H}$ NMR (400 MHz, $C D C l$ ) $: \delta 8.17(\mathrm{~s}, 1 \mathrm{H}), 7.84-7.93(\mathrm{~m}, 4 \mathrm{H}), 7.82(\mathrm{~d}, J=8.4 \mathrm{~Hz}, 1 \mathrm{H}), 7.56(\mathrm{t}, J=7.4 \mathrm{~Hz}, 1 \mathrm{H})$, $7.51(\mathrm{t}, J=7.3 \mathrm{~Hz}, 1 \mathrm{H}), 7.44$ (d, $J=9.1 \mathrm{~Hz}, 1 \mathrm{H}), 6.82$ (t, $J=7.8 \mathrm{~Hz}, 1 \mathrm{H}), 6.70(\mathrm{t}, J=6.8 \mathrm{~Hz}, 1 \mathrm{H}), 2.87$ $(\mathrm{t}, J=7.5 \mathrm{~Hz}, 2 \mathrm{H}), 2.26(\mathrm{~s}, 3 \mathrm{H}), 1.54-1.68(\mathrm{~m}, 2 \mathrm{H}), 1.30-1.42(\mathrm{~m}, 4 \mathrm{H}), 0.90(\mathrm{t}, J=6.3 \mathrm{~Hz}, 3 \mathrm{H}) ;{ }^{13} \mathrm{C}$ NMR (100 MHz, $\left.\mathrm{CDCl}_{3}\right): \delta$ 192.0, 139.5, 136.1, 134.7, 132.7, 129.6, 129.1, 128.0, 127.8, 127.3, 126.4, 125.7, 124.5, 124.3, 122.4, 121.2, 119.4, 112.5, 112.3, 31.6, 27.0, 23.5, 22.5, 14.1, 11.9; IR (neat): 3056, 2925, 2856, 1602, 1492, $1392 \mathrm{~cm}^{-1}$; HRMS (ESI) m/z 356.2017 [356.2009 calcd for $\left.\mathrm{C}_{25} \mathrm{H}_{26} \mathrm{NO}(\mathrm{M}+\mathrm{H})^{+}\right]$.

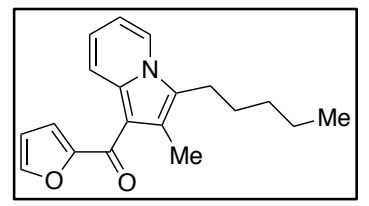

1-(2-furyl)-2-methyl-3-pentylindolizine (71). Following the general procedure outlined above for the synthesis of compound 4, catalyst $5 \mathrm{c}(25 \mathrm{mg}, 0.029 \mathrm{mmol})$, 1-phenylethanol $(0.3 \mathrm{~mL})$ and 2-furyl methyl ketone $(1.90 \mathrm{~g}, 17.0 \mathrm{mmol})$ were added to 2-(2-octynoxy)pyridine (1, $104 \mathrm{mg}, 0.51 \mathrm{mmol})$. After 18 hours, the reaction was worked up and purified by column chromatography $\left(\mathrm{SiO}_{2}, 85: 15\right.$ hexanes/ethyl acetate) to afford $107 \mathrm{mg}(71 \%$ yield) of $7 \mathrm{l}$ as a thick yellow oil. ${ }^{1} \mathrm{H}$ NMR (400 MHz, $\left.C D C l_{3}\right): \delta 7.82(\mathrm{~d}, J=6.8 \mathrm{~Hz}, 1 \mathrm{H}), 7.63(\mathrm{dt}, J=$ 1.2, $9.0 \mathrm{~Hz}, 1 \mathrm{H}), 7.58$ (q, $J=0.8 \mathrm{~Hz}, 1 \mathrm{H}), 7.08(\mathrm{dd}, J=0.8,3.6 \mathrm{~Hz}, 1 \mathrm{H}), 6.90$ (ddd, $J=1.0,6.6,9.0 \mathrm{~Hz}$, $1 \mathrm{H}), 6.69(\mathrm{td}, J=1.2,6.8 \mathrm{~Hz}, 1 \mathrm{H}), 6.54(\mathrm{t}, J=1.8,3.4 \mathrm{~Hz}, 1 \mathrm{H}), 2.84$ (t, $J=7.6 \mathrm{~Hz}, 2 \mathrm{H}), 2.33(\mathrm{~s}, 3 \mathrm{H})$, 1.52-1.63 (m, 2H), 1.30-1.39 (m, 4H), $0.89(\mathrm{t}, J=6.8 \mathrm{~Hz}, 3 \mathrm{H}) ;{ }^{13} \mathrm{C} \mathrm{NMR}\left(100 \mathrm{MHz}, \mathrm{CDCl}_{3}\right): \delta 178.5$, $154.4,145.0,135.4,124.5,123.6,122.4,120.9$, 119.1, 116.8, 112.2, 111.9, 31.6, 29.7, 26.9, 23.5, 22.5, 14.0, 11.3; IR (neat): 3112, 2924, 2855, 1735, 1599, 1495, 1392, $1248 \mathrm{~cm}^{-1}$; HRMS (ESI) m/z 296.1641 [296.1645 calcd for $\left.\mathrm{C}_{19} \mathrm{H}_{22} \mathrm{NO}_{2}(\mathrm{M}+\mathrm{H})^{+}\right]$.

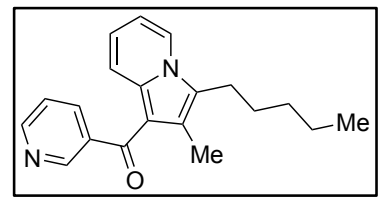

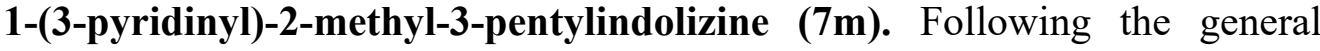
procedure outlined above for the synthesis of compound 4, catalyst $5 \mathrm{c}$ ( $23 \mathrm{mg}$, $0.026 \mathrm{mmol}), 1$-phenylethanol $(0.3 \mathrm{~mL})$ and 2-acetyl pyridine $(2.0 \mathrm{~mL})$ were added to 2-(2-octynoxy)pyridine (1, $110 \mathrm{mg}, 0.54 \mathrm{mmol})$. After 18 hours, the reaction was worked up and purified by column chromatography $\left(\mathrm{SiO}_{2}, 7: 3\right.$ hexanes/ethyl acetate) to afford $84 \mathrm{mg}$ (51\% yield) of $7 \mathbf{m}$ as a thick yellow powder. mp: $104-105{ }^{\circ} \mathrm{C} ;{ }^{1} \mathrm{H}$ NMR $\left(400 \mathrm{MHz}, C D C l_{3}\right): \delta 8.83(\mathrm{~s}, 1 \mathrm{H}), 8.71(\mathrm{~d}$, 
S12 Supporting Information. Rossler, Hartgerink, Zerull, Anderson et. al.

$J=4.8 \mathrm{~Hz}, 1 \mathrm{H}), 7.96(\mathrm{~d}, J=8.4 \mathrm{~Hz}, 1 \mathrm{H}), 7.86(\mathrm{~d}, J=6.8 \mathrm{~Hz}, 1 \mathrm{H}), 7.53(\mathrm{~d}, J=9.2 \mathrm{~Hz}, 1 \mathrm{H}), 7.38(\mathrm{dd}, J$ $=5.2,8.0 \mathrm{~Hz}, 1 \mathrm{H}), 6.94(\mathrm{dd}, J=6.8,8.8 \mathrm{~Hz}, 1 \mathrm{H}), 6.75(\mathrm{t}, J=6.8 \mathrm{~Hz}, 1 \mathrm{H}), 2.83(\mathrm{t}, J=7.6 \mathrm{~Hz}, 2 \mathrm{H}), 2.19$ $(\mathrm{s}, 3 \mathrm{H}), 1.57$ (pentet, $J=6.8 \mathrm{~Hz}, 2 \mathrm{H}), 1.29-1.37(\mathrm{~m}, 4 \mathrm{H}), 0.88$ (t, $J=6.4 \mathrm{~Hz}, 3 \mathrm{H}) ;{ }^{13} \mathrm{C} \mathrm{NMR}(100 \mathrm{MHz}$, $\left.\mathrm{CDCl}_{3}\right): \delta 189.3,151.4,149.9,137.7,136.5,136.0,125.0,124.1,123.4,122.7,122.4,119.2,112.8$, 112.0, 31.5, 26.9, 23.4, 22.5, 14.0, 12.1; IR (neat): 3089, 2917, 2852, 1589, $1486 \mathrm{~cm}^{-1}$; HRMS (ESI) m/z $307.1805\left[307.1805\right.$ calcd for $\left.\mathrm{C}_{20} \mathrm{H}_{23} \mathrm{~N}_{2} \mathrm{O}(\mathrm{M}+\mathrm{H})^{+}\right]$.

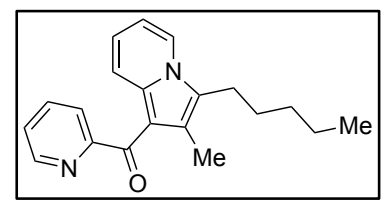

1-(2-pyridinyl)-2-methyl-3-pentylindolizine (7n). Following the general procedure outlined above for the synthesis of compound 4, catalyst $5 \mathbf{c}(25 \mathrm{mg}$, $0.028 \mathrm{mmol}), 1$-phenylethanol $(0.3 \mathrm{~mL})$ and 3-acetyl pyridine $(2.0 \mathrm{~mL})$ were added to 2-(2-octynoxy)pyridine $(\mathbf{1}, 111 \mathrm{mg}, 0.55 \mathrm{mmol})$. After $18 \mathrm{hours}$, the reaction was worked up and purified by column chromatography $\left(\mathrm{SiO}_{2}, 7: 3\right.$ hexanes/ethyl acetate) to afford $99 \mathrm{mg}(59 \%$ yield) of $7 \mathbf{n}$ as a thick yellow oil. ${ }^{1} \mathrm{H}$ NMR $\left(400 \mathrm{MHz}, C D C l_{3}\right): \delta 8.61(\mathrm{~d}, J=4.4 \mathrm{~Hz}, 1 \mathrm{H}), 7.76-7.87(\mathrm{~m}, 3 \mathrm{H})$, $7.50(\mathrm{~d}, J=9.2 \mathrm{~Hz}, 1 \mathrm{H}), 7.38 \mathrm{dd}, J=4.8,7.2 \mathrm{~Hz}, 1 \mathrm{H}), 6.91(\mathrm{t}, J=6.8 \mathrm{~Hz}, 1 \mathrm{H}), 6.70(\mathrm{t}, J=6.8 \mathrm{~Hz}, 1 \mathrm{H})$, $2.80(\mathrm{t}, J=7.6 \mathrm{~Hz}, 2 \mathrm{H}), 2.13(\mathrm{~s}, 3 \mathrm{H}), 1.48-1.58(\mathrm{~m}, 2 \mathrm{H}), 1.27-1.36(\mathrm{~m}, 4 \mathrm{H}), 0.86(\mathrm{t}, J=6.4 \mathrm{~Hz}, 3 \mathrm{H}) ;{ }^{13} \mathrm{C}$ NMR (100 MHz, $\left.\mathrm{CDCl}_{3}\right): \delta 189.9,159.1,148.6,137.01,136.96,125.0,124.9,124.5,123.1,122.6$, 122.2, 119.6, 112.7, 111.5, 31.6, 26.9, 23.4, 22.5, 14.0, 11.3; IR (neat): 3049, 2923, 2855, 1602, 1488, $1391 \mathrm{~cm}^{-1}$; HRMS (ESI) m/z 307.1805 [307.1805 calcd for $\left.\mathrm{C}_{20} \mathrm{H}_{23} \mathrm{~N}_{2} \mathrm{O}(\mathrm{M}+\mathrm{H})^{+}\right]$.

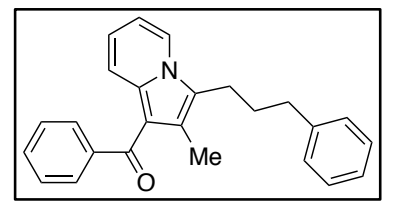

1-benzoyl-2-methyl-3-(3-phenylpropyl)indolizine (9a). Following the general procedure outlined above for the synthesis of compound 4 , catalyst 5c (25 mg, $0.029 \mathrm{mmol}), 1$-phenylethanol $(0.33 \mathrm{~mL})$ and acetophenone $(2.16 \mathrm{~mL})$ were added to 2-(6-phenyl-2-hexynoxy)pyridine (8a, $136 \mathrm{mg}, 0.54 \mathrm{mmol})$. After 18 hours, the reaction was worked up and purified by column chromatography $\left(\mathrm{SiO}_{2}, 85: 15\right.$ hexanes/ethyl acetate) to afford 94 mg (49\% yield) of 9a as a thick yellow oil. ${ }^{1} \mathrm{H}$ NMR (400 MHz, $\left.C D C l_{3}\right): \delta 7.68($ app t, $J=8.9 \mathrm{~Hz}, 3 \mathrm{H})$, $7.50(\mathrm{t}, J=7.3 \mathrm{~Hz}, 1 \mathrm{H}), 7.42(\operatorname{app~t}, J=6.1 \mathrm{~Hz}, 3 \mathrm{H}), 7.29(\mathrm{t}, J=7.4 \mathrm{~Hz}, 2 \mathrm{H}), 7.20(\operatorname{app~t}, J=6.9 \mathrm{~Hz}$, $3 \mathrm{H}), 6.65(\mathrm{t}, J=7.8 \mathrm{~Hz}, 1 \mathrm{H}), 6.66(\mathrm{t}, J=6.8 \mathrm{~Hz}, 1 \mathrm{H}), 2.88(\mathrm{t}, J=7.6 \mathrm{~Hz}, 2 \mathrm{H}), 2.71(\mathrm{t}, J=7.5 \mathrm{~Hz}, 2 \mathrm{H})$, 2.22 (s, 3H), 1.92 (pentet, $J=7.6 \mathrm{~Hz}, 2 \mathrm{H}) ;{ }^{13} \mathrm{C} \mathrm{NMR}\left(100 \mathrm{MHz}, \mathrm{CDCl}_{3}\right): \delta 192.1,142.3,141.4,136.1$, $130.9,128.7,128.4,128.3,128.2,126.0,124.4,123.9,122.3,121.3,119.4,112.32,112.28,35.4,28.7$, 22.9, 11.8; IR (neat): 3059, 3024, 2926, 2856, 1608, 1495, 1393, $1240 \mathrm{~cm}^{-1}$; HRMS (ESI) m/z 354.1861 [354.1852 calcd for $\left.\mathrm{C}_{25} \mathrm{H}_{24} \mathrm{NO}(\mathrm{M}+\mathrm{H})^{+}\right]$. 
S13 Supporting Information. Rossler, Hartgerink, Zerull, Anderson et. al.

2-methyl-1-(4-nitrobenzoyl)-3-(3-phenylpropyl)indolizine (9aa). Following the general procedure

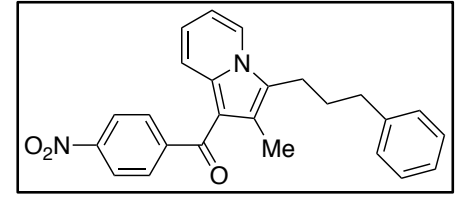
outlined above for the synthesis of compound 4, catalyst 5c (16 mg, 0.018 mmol), 1-phenylethanol (0.21 mL) and 4'-nitroacetophenone (1.99 g, 12.0 mmol) were added to 2-(6-phenyl-2-hexynoxy)pyridine (8a, $88 \mathrm{mg}, 0.35$ mmol). After 18 hours, the reaction was worked up and purified by column chromatography $\left(\mathrm{SiO}_{2}, 85: 15\right.$ hexanes/ethyl acetate) to afford $100 \mathrm{mg}$ (72\% yield) of 9aa as a thick red oil. ${ }^{1} \mathrm{H}$ NMR $(400 \mathrm{MHz}$, $\left.C D l_{3}\right): \delta 8.29(\mathrm{~d}, J=8.0 \mathrm{~Hz}, 2 \mathrm{H}), 7.77(\mathrm{~d}, J=8.2 \mathrm{~Hz}, 2 \mathrm{H}), 7.73(\mathrm{~d}, J=6.8 \mathrm{~Hz}, 1 \mathrm{H}), 7.50(\mathrm{~d}, J=8.9$ $\mathrm{Hz}, 1 \mathrm{H}), 7.29$ (t, $J=7.2 \mathrm{~Hz}, 2 \mathrm{H}), 7.19(\operatorname{app~t}, J=8.9 \mathrm{~Hz}, 3 \mathrm{H}), 6.97$ (t, $J=7.8 \mathrm{~Hz}, 1 \mathrm{H}), 6.74(\mathrm{t}, J=6.8$ $\mathrm{Hz}, 1 \mathrm{H}), 2.88$ (t, $J=7.6 \mathrm{~Hz}, 2 \mathrm{H}), 2.71$ (t, $J=7.4 \mathrm{~Hz}, 2 \mathrm{H}), 2.14$ (s, 3H), 1.92 (pentet, $J=7.5 \mathrm{~Hz}, 2 \mathrm{H})$; ${ }^{13} \mathrm{C}$ NMR $\left(100 \mathrm{MHz}, \mathrm{CDCl}_{3}\right): \delta 189.3,148.9,148.1,141.2,136.8,129.3,128.5,128.3,126.1,124.7$, 124.3, 123.6, 122.8, 122.7, 119.2, 113.1, 111.5, 35.4, 28.6, 22.8, 12.1; IR (neat): 3025, 2926, 2860, 1595 , 1493, $1345 \mathrm{~cm}^{-1}$; HRMS (ESI) m/z 399.1692 [399.1703 calcd for $\mathrm{C}_{25} \mathrm{H}_{23} \mathrm{~N}_{2} \mathrm{O}_{3}(\mathrm{M}+\mathrm{H})^{+}$].

1-benzoyl-2-methyl-3-(2-phenylethyl)indolizine (9b). Following the general procedure outlined above

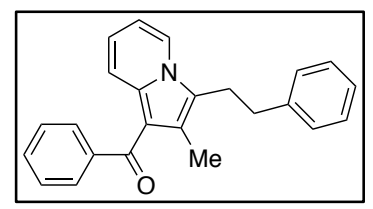
for the synthesis of compound 4, catalyst 5c (25 mg, $0.028 \mathrm{mmol})$, 1phenylethanol $(0.31 \mathrm{~mL})$ and acetophenone $(2.1 \mathrm{~mL})$ were added to $2-(5$-phenyl2-pentynoxy)pyridine $(\mathbf{8 b}, 122 \mathrm{mg}, 0.52 \mathrm{mmol})$. After 18 hours, the reaction was worked up and purified by column chromatography ( $\mathrm{SiO}_{2}, 85: 15$ hexanes/ethyl acetate) to afford $86 \mathrm{mg}$ (49\% yield) of 9b as a thick yellow oil. ${ }^{1} \mathrm{H}$ NMR (400 MHz, $\left.C D C l_{3}\right): \delta 7.79(\mathrm{~d}, J=6.9 \mathrm{~Hz}, 1 \mathrm{H}), 7.64(\mathrm{~d}$, $J=7.0 \mathrm{~Hz}, 2 \mathrm{H}), 7.50(\mathrm{dd}, J=8.8,13.6 \mathrm{~Hz}, 2 \mathrm{H}), 7.41(\mathrm{t}, J=7.4 \mathrm{~Hz}, 2 \mathrm{H}), 7.17-7.28(\mathrm{~m}, 3 \mathrm{H}), 7.07$ (d, $J$ $=7.0 \mathrm{~Hz}, 2 \mathrm{H}), 6.88(\mathrm{t}, J=7.8 \mathrm{~Hz}, 1 \mathrm{H}), 6.68(\mathrm{t}, J=6.8 \mathrm{~Hz}, 1 \mathrm{H}), 3.15(\mathrm{t}, J=7.5 \mathrm{~Hz}, 2 \mathrm{H}), 2.87(\mathrm{t}, J=7.4$ $\mathrm{Hz}, 2 \mathrm{H}), 1.97$ (s, 3H); ${ }^{13} \mathrm{C}$ NMR (100 MHz, $\left.\mathrm{CDCl}_{3}\right): \delta 192.2,142.1,140.7,136.1,130.9,128.8,128.48$, 128.46, 128.2 126.3, 124.8, 123.0, 122.0, 121.4, 119.4, 112.34, 112.28, 33.6, 25.8, 11.5; IR (neat): 3059, 3024, 2922, 2856, 1608, 1494, 1393, $1240 \mathrm{~cm}^{-1}$; HRMS (ESI) m/z 340.1690 [340.1696 calcd for $\left.\mathrm{C}_{22} \mathrm{H}_{22} \mathrm{NO}(\mathrm{M}+\mathrm{H})^{+}\right]$.

2-methyl-3-(3-phenylethyl)-1-(3,5-bistrifluoromethylbenzoyl)indolizine (9bb). Following the

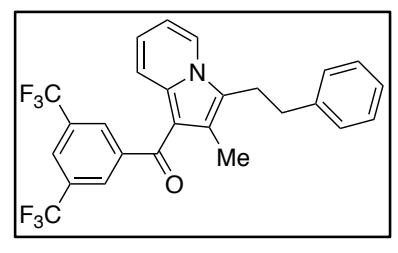
general procedure outlined above for the synthesis of compound 4 , catalyst $5 \mathrm{c}$ $(18 \mathrm{mg}, \quad 0.020 \mathrm{mmol})$, 1-phenylethanol $(0.22 \mathrm{~mL})$ and 3',5'bistrifluoromethylacetophenone $(1.48 \mathrm{~mL})$ were added to 2-(5-phenyl-2pentynoxy)pyridine $(\mathbf{8 b}, 87 \mathrm{mg}, 0.37 \mathrm{mmol})$. After 18 hours, the reaction was worked up and purified by column chromatography $\left(\mathrm{SiO}_{2}, 9: 1\right.$ hexanes/ethyl acetate) to afford $113 \mathrm{mg}$ 
S14 Supporting Information. Rossler, Hartgerink, Zerull, Anderson et. al.

(64\% yield) of $9 \mathbf{b b}$ as a yellow powder. mp: $96-97{ }^{\circ} \mathrm{C} ;{ }^{1} \mathrm{H}$ NMR $\left(400 \mathrm{MHz}, C D C l_{3}\right): \delta 8.07(\mathrm{~s}, 2 \mathrm{H})$, 7.99 (s, 1H), 7.86 (d, $J=6.8 \mathrm{~Hz}, 1 \mathrm{H}), 7.73$ (d, $J=9.0 \mathrm{~Hz}, 1 \mathrm{H}), 7.23$ (app q, $J=8.1 \mathrm{~Hz}, 3 \mathrm{H}), 7.01-7.08$ $(\mathrm{m}, 3 \mathrm{H}), 6.80(\mathrm{t}, J=6.8 \mathrm{~Hz}, 1 \mathrm{H}), 3.16(\mathrm{t}, J=7.4 \mathrm{~Hz}, 2 \mathrm{H}), 2.89(\mathrm{t}, J=7.4 \mathrm{~Hz}, 2 \mathrm{H}), 1.83(\mathrm{~s}, 3 \mathrm{H}) ;{ }^{13} \mathrm{C}$ NMR (100 MHz, $\left.\mathrm{CDCl}_{3}\right): \delta 187.9,143.9,140.3,137.0,131.6$ (q, $\left.J=34 \mathrm{~Hz}\right), 128.9$ (q, $J=3.0 \mathrm{~Hz}$ ), $128.5,128.4,126.5,124.4,124.0$ (heptet, $J=4.0 \mathrm{~Hz}$ ), 123.9, 123.2, 123.1 (q, $J=271 \mathrm{~Hz}$ ), 122.5, 119.3, 113.3, 111.1, 33.4, 25.8, 11.8; IR (neat): 3066, 2918, 2851, 1598, 1491, $1368 \mathrm{~cm}^{-1}$; HRMS (ESI) m/z 476.1433 [476.1444 calcd for $\left.\mathrm{C}_{26} \mathrm{H}_{20} \mathrm{~F}_{6} \mathrm{NO}(\mathrm{M}+\mathrm{H})^{+}\right]$.

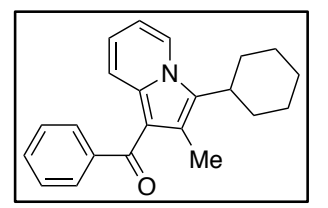

1-benzoyl-3-cyclohexyl-2-methylindolizine (9c). Following the general procedure outlined above for the synthesis of compound 4, catalyst $5 \mathbf{c}(23 \mathrm{mg}, 0.026 \mathrm{mmol})$, 1-phenylethanol $(0.3 \mathrm{~mL})$ and acetophenone $(2.0 \mathrm{~mL})$ were added to 2-(3cyclohexyl-2-propynoxy)pyridine (8c, $107 \mathrm{mg}, 0.50 \mathrm{mmol})$. After 18 hours, the reaction was worked up and purified by column chromatography $\left(\mathrm{SiO}_{2}, 19: 1\right.$ to 9:1 hexanes/ethyl acetate) to afford $63 \mathrm{mg}(40 \%$ yield) of 9c as a yellow solid. mp: $120-123{ }^{\circ} \mathrm{C} ;{ }^{1} \mathrm{H}$ NMR (400 MHz, $\left.C D C l 3\right): \delta 8.03(\mathrm{~d}, J=6.8 \mathrm{~Hz}, 1 \mathrm{H})$, $7.69(\mathrm{~d}, J=7.8 \mathrm{~Hz}, 2 \mathrm{H}), 7.49$ (app t, $J=6.8 \mathrm{~Hz}, 1 \mathrm{H}), 7.41(\operatorname{app~t}, J=7.5 \mathrm{~Hz}, 2 \mathrm{H}), 7.36$ (d, $J=9.0 \mathrm{~Hz}$, $1 \mathrm{H}), 6.80(\mathrm{t}, J=7.8 \mathrm{~Hz}, 1 \mathrm{H}), 6.64(\mathrm{t}, J=6.7 \mathrm{~Hz}, 1 \mathrm{H}), 3.01(\mathrm{t}, J=11.4 \mathrm{~Hz}, 1 \mathrm{H}), 2.31$ (s, 3H), 1.73-1.98 (m, 7H), 1.28-1.50 (m, 3H); $\left.{ }^{13} \mathrm{C} \mathrm{NMR} \mathrm{(100} \mathrm{MHz,} \mathrm{CDCl}_{3}\right): \delta$ 192.3, 142.2, 135.9, 131.0, 129.0, 128.2, 128.0, 123.5, 123.1, 120.6, 119.4, 112.9, 11.8, 36.0, 29.9, 27.2, 26.1, 12.5; IR (neat): 2925, 2851, 1607, 1495, 1392, $1239 \mathrm{~cm}^{-1}$; HRMS (ESI) m/z 318.1851 [318.1852 calcd for $\mathrm{C}_{22} \mathrm{H}_{24} \mathrm{NO}(\mathrm{M}+\mathrm{H})^{+}$].

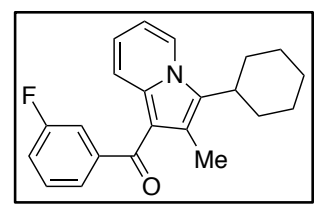

3-cyclohexyl-1-(3-fluorobenzoyl)-2-methylindolizine (9cc). Following the general procedure outlined above for the synthesis of compound 4, catalyst 5 c (20 $\mathrm{mg}, 0.023 \mathrm{mmol})$, 1-phenylethanol $(0.28 \mathrm{~mL})$ and 3'-fluoroacetophenone (1.84 mL) were added to 2-(3-cyclohexyl-2-propynoxy)pyridine $(\mathbf{8 c}, 100 \mathrm{mg}, 0.46 \mathrm{mmol})$. After 18 hours, the reaction was worked up and purified by column chromatography $\left(\mathrm{SiO}_{2}, 19: 1\right.$ hexanes/ethyl acetate) to afford $88 \mathrm{mg}$ (57\% yield) of $9 \mathrm{c}$ as a yellow solid. mp: $95-96{ }^{\circ} \mathrm{C} ;{ }^{1} \mathrm{H} \mathrm{NMR}\left(400 \mathrm{MHz}, C D C l_{3}\right): \delta 8.04$ (d, $J=6.7 \mathrm{~Hz}, 1 \mathrm{H}), 7.46$ (d, $J=7.5 \mathrm{~Hz}, 1 \mathrm{H}), 7.39$ (t, $J=8.9 \mathrm{~Hz}, 3 \mathrm{H}), 7.19$ (t, $J=8.2 \mathrm{~Hz}, 1 \mathrm{H}), 6.84(\mathrm{t}, J=$ $7.7 \mathrm{~Hz}, 1 \mathrm{H}), 6.67$ (t, $J=6.8 \mathrm{~Hz}, 1 \mathrm{H}), 3.01$ (t, $J=11.9 \mathrm{~Hz}, 1 \mathrm{H}), 2.29$ (s, 3H), 1.77-1.98 (m, 6H), 1.21$1.51(\mathrm{~m}, 4 \mathrm{H}) ;{ }^{13} \mathrm{C} \mathrm{NMR}\left(100 \mathrm{MHz}, \mathrm{CDCl}_{3}\right): \delta 190.5(\mathrm{~d}, J=2.0 \mathrm{~Hz}), 163.8,161.4,144.4(\mathrm{~d}, J=6.0 \mathrm{~Hz})$, $136.1,129.8(\mathrm{~d}, J=7.7 \mathrm{~Hz}), 128.3,124.6(\mathrm{~d}, J=2.9 \mathrm{~Hz}), 123.4(\mathrm{~d}, J=19 \mathrm{~Hz}), 121.2,119.2,117.8(\mathrm{~d}, J$ $=12 \mathrm{~Hz}), 115.7$ (d, $J=22 \mathrm{~Hz}), 112.5,112.1,36.0,29.8,27.1,26.1,12.5$; IR (neat): 3102, 2919, 2850, $1628,1601,1494,1246 \mathrm{~cm}^{-1}$; HRMS (ESI) m/z 336.1743 [336.1758 calcd for $\left.\mathrm{C}_{22} \mathrm{H}_{23} \mathrm{FNO}(\mathrm{M}+\mathrm{H})^{+}\right]$. 
S15 Supporting Information. Rossler, Hartgerink, Zerull, Anderson et. al.

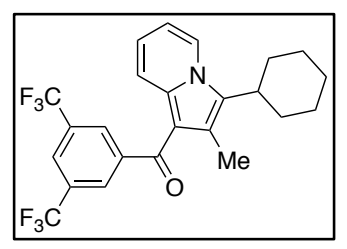

\section{3-cyclohexyl-2-methyl-1-(3,5-bistrifluoromethylbenzoyl)indolizine (9cce).}

Following the general procedure outlined above for the synthesis of compound 4 , catalyst 5c (26 mg, $0.029 \mathrm{mmol})$, 1-phenylethanol $(0.33 \mathrm{~mL})$ and 3',5'bistrifluoromethylacetophenone $(2.2 \mathrm{~mL})$ were added to 2-(3-cyclohexyl-2propynoxy)pyridine $(\mathbf{8 c}, 118 \mathrm{mg}, 0.55 \mathrm{mmol})$. After 18 hours, the reaction was worked up and purified by column chromatography ( $\mathrm{SiO}_{2}, 19: 1$ hexanes/ethyl acetate) to afford $177 \mathrm{mg}$ (71\% yield) of 9ccc as a yellow solid. mp: $134-137{ }^{\circ} \mathrm{C} ;{ }^{1} \mathrm{H}$ NMR $\left(400 \mathrm{MHz}, C D C l_{3}\right): \delta 8.14(\mathrm{~s}, 1 \mathrm{H}), 8.11(\mathrm{~d}, J=6.7 \mathrm{~Hz}, 1 \mathrm{H})$, $8.00(\mathrm{~s}, 1 \mathrm{H}), 7.53(\mathrm{~d}, J=8.9 \mathrm{~Hz}, 1 \mathrm{H}), 6.95(\mathrm{t}, J=7.8 \mathrm{~Hz}, 1 \mathrm{H}), 6.75(\mathrm{t}, J=6.8 \mathrm{~Hz}, 1 \mathrm{H}), 3.03(\mathrm{t}, J=10.9$ $\mathrm{Hz}, 1 \mathrm{H}), 2.22(\mathrm{~s}, 3 \mathrm{H}), 1.76-1.99(\mathrm{~m}, 6 \mathrm{H}), 1.23-1.51(\mathrm{~m}, 4 \mathrm{H}) ;{ }^{13} \mathrm{C} \mathrm{NMR}\left(100 \mathrm{MHz}, \mathrm{CDCl}_{3}\right): \delta 188.0$, $143.9,136.7,131.7(\mathrm{q}, J=34 \mathrm{~Hz}), 129.2(\mathrm{q}, J=3.6 \mathrm{~Hz}), 128.9,124.1(\mathrm{q}, J=3.8 \mathrm{~Hz}), 123.7,123.11(\mathrm{q}$, $J=272 \mathrm{~Hz}$ ), 123.09, 122.4, 119.0, 112.8, 111.7, 36.1, 29.8, 27.1, 26.0, 12.8; IR (neat): 2930, 2856, 1626, 1494, 1396, 1275, $1127 \mathrm{~cm}^{-1}$; HRMS (ESI) m/z 454.1592 [454.1600 calcd for $\mathrm{C}_{24} \mathrm{H}_{22} \mathrm{~F}_{6} \mathrm{NO}(\mathrm{M}+\mathrm{H})^{+}$].

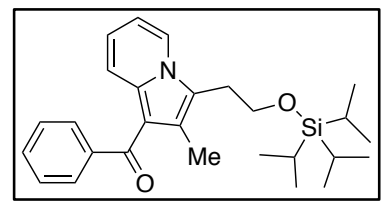

1-benzoyl-2-methyl-3-(2-triisopropylsiloxyethyl)indolizine (9d). Following the general procedure outlined above for the synthesis of compound 4, catalyst 5c (15 mg, $0.017 \mathrm{mmol}), 1-$ phenylethanol $(0.21 \mathrm{~mL})$ and acetophenone $(1.4 \mathrm{~mL})$ were added to 2-(5-triisopropylsiloxy-2-pentynoxy)pyridine (8d, $117 \mathrm{mg}, 0.35 \mathrm{mmol})$. After 18 hours, the reaction was worked up and purified by column chromatography $\left(\mathrm{SiO}_{2}, 19: 1\right.$ to 9:1 hexanes/ethyl acetate) to afford $68 \mathrm{mg}$ (45\% yield) of 9d as a thick yellow oil. ${ }^{1} \mathrm{H}$ NMR (400 MHz, $\left.C D C l 3\right): \delta 8.07$ (d, $J=6.6 \mathrm{~Hz}, 1 \mathrm{H}), 7.65(\mathrm{~d}, J=7.6 \mathrm{~Hz}, 2 \mathrm{H}), 7.49(\mathrm{t}, J=7.2 \mathrm{~Hz}, 1 \mathrm{H}), 7.41($ app t, $J=9.1 \mathrm{~Hz}, 3 \mathrm{H}), 6.84$ (t, $J=7.9 \mathrm{~Hz}, 1 \mathrm{H}), 6.65(\mathrm{t}, J=6.7 \mathrm{~Hz}, 1 \mathrm{H}), 3.90(\mathrm{t}, J=6.2 \mathrm{~Hz}, 2 \mathrm{H}), 3.12(\mathrm{t}, J=6.1 \mathrm{~Hz}, 2 \mathrm{H}), 2.24(\mathrm{~s}, 3 \mathrm{H})$, 0.89-1.08 (m, 21H); ${ }^{13} \mathrm{C}$ NMR (100 MHz, $\left.\mathrm{CDCl}_{3}\right): \delta$ 192.1, 142.3, 136.3, 130.8, 128.7, 128.2, 125.1, 123.2, 122.1, 121.4, 119.1, 112.3, 112.0, 62.2, 27.3, 17.8, 11.80, 11.76; IR (neat): 2941, 2864, 1611, 1496, 1394, $1138 \mathrm{~cm}^{-1}$; HRMS (ESI) m/z 436.2653 [436.2666 calcd for $\mathrm{C}_{27} \mathrm{H}_{38} \mathrm{NO}_{2} \mathrm{Si}(\mathrm{M}+\mathrm{H})^{+}$].

1-(3-fluorobenzoyl)-2-methyl-3-(2-triisopropylsiloxyethyl)indolizine (9dd). Following the general

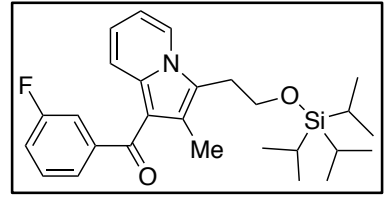
procedure outlined above for the synthesis of compound 4, catalyst $5 \mathrm{c}(17 \mathrm{mg}$, $0.018 \mathrm{mmol}), 1$-phenylethanol $(0.20 \mathrm{~mL})$ and 3'-fluoroacetophenone $(1.4 \mathrm{~mL})$ were added to 2-(5-triisopropylsiloxy-2-pentynoxy)pyridine (8d, $110 \mathrm{mg}, 0.33$ mmol). After 18 hours, the reaction was worked up and purified by column chromatography $\left(\mathrm{SiO}_{2}, 19: 1\right.$ to 9:1 hexanes/ethyl acetate) to afford $88 \mathrm{mg}$ (59\% yield) of 9dd as a thick yellow oil. ${ }^{1} \mathrm{H}$ NMR (400 MHz, $C D C l 3): \delta 8.09(\mathrm{~d}, J=6.7 \mathrm{~Hz}, 1 \mathrm{H}), 7.30-7.47(\mathrm{~m}, 4 \mathrm{H}), 7.18(\mathrm{t}, J=8.1 \mathrm{~Hz}, 1 \mathrm{H}), 6.89(\mathrm{t}, J=7.9$ 
S16 Supporting Information. Rossler, Hartgerink, Zerull, Anderson et. al.

$\mathrm{Hz}, 1 \mathrm{H}), 6.68(\mathrm{t}, J=6.7 \mathrm{~Hz}, 1 \mathrm{H}), 3.91(\mathrm{t}, J=6.1 \mathrm{~Hz}, 2 \mathrm{H}), 3.11(\mathrm{t}, J=6.0 \mathrm{~Hz}, 2 \mathrm{H}), 2.22(\mathrm{~s}, 3 \mathrm{H}), 0.87-$ 1.07 (m, 21H); ${ }^{13} \mathrm{C}$ NMR (100 MHz, $\left.\mathrm{CDCl}_{3}\right): \delta 190.3$ (d, $\left.J=2 \mathrm{~Hz}\right), 162.6$ (d, $\left.J=246 \mathrm{~Hz}\right), 144.5(\mathrm{~d}, J=$ $6.0 \mathrm{~Hz}), 136.5,129.8(\mathrm{~d}, J=7.7 \mathrm{~Hz}), 125.0,124.3(\mathrm{~d}, J=3.0 \mathrm{~Hz}), 123.4,122.5,122.0,119.0,117.6(\mathrm{~d}$, $J=21 \mathrm{~Hz}), 115.5(\mathrm{~d}, J=22 \mathrm{~Hz}), 112.2,111.8,62.3,27.2,17.8,11.8$ (2C); IR (neat): 2941, 2864, 1605, 1494, 1393, 1252, $1101 \mathrm{~cm}^{-1}$; HRMS (ESI) m/z 454.2575 [454.2572 calcd for $\mathrm{C}_{27} \mathrm{H}_{37} \mathrm{FNO}_{2} \mathrm{Si}(\mathrm{M}+\mathrm{H})^{+}$].

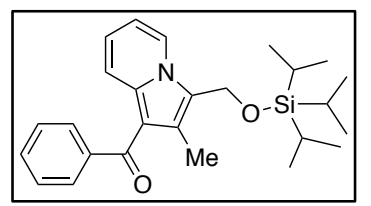

1-benzoyl-2-methyl-3-triisopropylsiloxymethylindolizine (9e). Following the general procedure outlined above for the synthesis of compound 4 , catalyst 5c (16 mg, $0.018 \mathrm{mmol})$, 1-phenylethanol $(0.20 \mathrm{~mL})$ and acetophenone $(1.4 \mathrm{~mL})$ were added to 2-(4-triisopropylsiloxy-2-butynoxy)pyridine (8e, $108 \mathrm{mg}, 0.34 \mathrm{mmol})$. After 18 hours, the reaction was worked up and purified by column chromatography ( $\mathrm{SiO}_{2}, 19: 1$ to 9:1 to 85:15 hexanes/ethyl acetate) to afford $8 \mathrm{mg}\left(6 \%\right.$ yield) of $9 \mathrm{e}$ as a thick yellow oil. ${ }^{1} \mathrm{H} \mathrm{NMR}\left(400 \mathrm{MHz}, \mathrm{CDCl}_{3}\right)$ : $\delta 8.26(\mathrm{~d}, J=6.9 \mathrm{~Hz}, 1 \mathrm{H}), 7.66(\mathrm{~d}, J=7.8 \mathrm{~Hz}, 2 \mathrm{H}), 7.50(\mathrm{t}, J=6.9 \mathrm{~Hz}, 1 \mathrm{H}), 7.36-7.46(\mathrm{~m}, 3 \mathrm{H}), 6.92(\mathrm{t}$, $J=7.7 \mathrm{~Hz}, 1 \mathrm{H}), 6.72(\mathrm{t}, J=6.6 \mathrm{~Hz}, 1 \mathrm{H}), 5.02(\mathrm{~s}, 2 \mathrm{H}), 2.27(\mathrm{~s}, 3 \mathrm{H}), 1.15$ (heptet, $J=7.5 \mathrm{~Hz}, 3 \mathrm{H}), 1.05$ (app d, $J=7.1 \mathrm{~Hz}, 18 \mathrm{H}) ;{ }^{13} \mathrm{C} \mathrm{NMR}\left(100 \mathrm{MHz}, \mathrm{CDCl}_{3}\right): \delta 192.2,142.2,136.8,130.9,128.7,128.2,125.2$, 124.5, 122.9, 122.6, 119.0, 112.2, 112.1, 54.9, 18.0, 12.0, 11.8; IR (neat): 2941, 2864, 1614, 1499, 1393 , 1240, $1058 \mathrm{~cm}^{-1}$; HRMS (ESI) m/z 422.2495 [422.2510 calcd for $\mathrm{C}_{26} \mathrm{H}_{36} \mathrm{NO}_{2} \mathrm{Si}(\mathrm{M}+\mathrm{H})^{+}$].

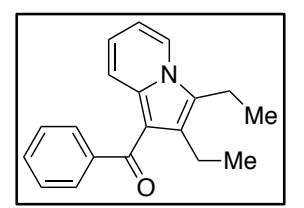

1-benzoyl-2,3-diethylindolizine (9f). Following the general procedure outlined above for the synthesis of compound 4, catalyst 5c (25 mg, $0.028 \mathrm{mmol}), 1$ phenylethanol $(0.3 \mathrm{~mL})$ and acetophenone $(2.0 \mathrm{~mL})$ were added to 2-(3-hexyn-2oxy)pyridine (8f, $91 \mathrm{mg}, 0.52 \mathrm{mmol})$. After 18 hours, the reaction was worked up and purified by column chromatography $\left(\mathrm{SiO}_{2}, 9: 1\right.$ hexanes/ethyl acetate) to afford $53 \mathrm{mg}$ (37\% yield) of 9f as a thick yellow oil. ${ }^{1} \mathrm{H}$ NMR (400 MHz, $\left.C D C l_{3}\right): \delta 7.84(\mathrm{~d}, J=6.7 \mathrm{~Hz}, 1 \mathrm{H}), 7.69(\mathrm{~d}, J=7.6 \mathrm{~Hz}, 2 \mathrm{H}), 7.50(\mathrm{t}, J=6.8 \mathrm{~Hz}$, 1H), $7.42(\mathrm{t}, J=7.5 \mathrm{~Hz}, 2 \mathrm{H}), 7.14(\mathrm{~d}, J=9.0 \mathrm{~Hz}, 1 \mathrm{H}), 6.78(\mathrm{t}, J=7.8 \mathrm{~Hz}, 1 \mathrm{H}), 6.67(\mathrm{t}, J=6.7 \mathrm{~Hz}, 1 \mathrm{H})$, $2.90(\mathrm{q}, J=7.4 \mathrm{~Hz}, 2 \mathrm{H}), 2.81(\mathrm{q}, J=7.3 \mathrm{~Hz}, 2 \mathrm{H}), 1.23(\mathrm{t}, J=7.3 \mathrm{~Hz}, 3 \mathrm{H}), 1.15(\mathrm{t}, J=7.3 \mathrm{~Hz}, 3 \mathrm{H}) ;{ }^{13} \mathrm{C}$ NMR (100 MHz, $\left.\mathrm{CDCl}_{3}\right): \delta 192.0,142.2,136.0,130.9,130.8,128.8,128.2,125.3,122.4,120.8,119.4$, 112.0, 111.4, 18.6, 16.7, 16.4, 12.3; IR (neat): 3057, 2965, 2871, 1608, 1494, 1389, 1306, $1236 \mathrm{~cm}^{-1}$; HRMS (ESI) m/z 278.1544 [278.1539 calcd for $\left.\mathrm{C}_{19} \mathrm{H}_{20} \mathrm{NO}(\mathrm{M}+\mathrm{H})^{+}\right]$. 
S17 Supporting Information. Rossler, Hartgerink, Zerull, Anderson et. al.

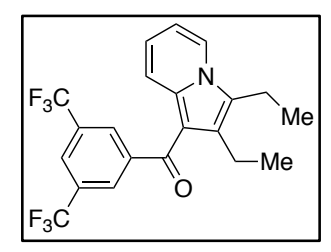

2,3-diethyl-1-(3,5-bistrifluoromethylbenzoyl)indolizine (9ff). Following the general procedure outlined above for the synthesis of compound 4 , catalyst $5 \mathrm{c}$ ( 25 mg, 0.028 mmol), 1-phenylethanol $(0.32 \mathrm{~mL})$ and 3',5'bistrifluromethylacetophenone $(1.7 \mathrm{~mL})$ were added to 2-(3-hexyn-2-oxy)pyridine (8f, $93 \mathrm{mg}, 0.53 \mathrm{mmol}$ ). After 18 hours, the reaction was worked up and purified by column chromatography $\left(\mathrm{SiO}_{2}, 9: 1\right.$ hexanes/ethyl acetate) to afford $151 \mathrm{mg}$ (69\% yield) of 9ff as a yellow powder. mp: $110-11{ }^{\circ} \mathrm{C} ;{ }^{1} \mathrm{H}$ NMR $\left(400 \mathrm{MHz}, C D C l_{3}\right): \delta 8.14(\mathrm{~s}, 2 \mathrm{H}), 8.01(\mathrm{~s}, 1 \mathrm{H}), 7.91(\mathrm{~d}, J=6.9 \mathrm{~Hz}$, 1H), $7.22(\mathrm{~d}, J=9.0 \mathrm{~Hz}, 1 \mathrm{H}), 6.93(\mathrm{t}, J=7.8 \mathrm{~Hz}, 1 \mathrm{H}), 6.78(\mathrm{t}, J=6.7 \mathrm{~Hz}, 1 \mathrm{H}), 2.92(\mathrm{q}, J=7.5 \mathrm{~Hz}, 2 \mathrm{H})$, $2.73(\mathrm{q}, J=7.4 \mathrm{~Hz}, 2 \mathrm{H}), 1.25(\mathrm{t}, J=7.5 \mathrm{~Hz}, 3 \mathrm{H}), 1.12$ (t, $J=7.4 \mathrm{~Hz}, 3 \mathrm{H}) ;{ }^{13} \mathrm{C} \mathrm{NMR}\left(100 \mathrm{MHz}, \mathrm{CDCl}_{3}\right)$ : $\delta 187.7,143.9,136.6,131.7(\mathrm{q}, J=34 \mathrm{~Hz}), 130.8,129.0(\mathrm{q}, J=3.0 \mathrm{~Hz}), 126.2,124.1$ (heptet, $J=3.8$ Hz), $123.1(\mathrm{q}, J=271 \mathrm{~Hz}), 122.9,122.5,118.8,112.9,110.1,18.7,16.7,16.2,12.2$; IR (neat): 3097, 2969, 2872, 1628, 1497, 1276, $1127 \mathrm{~cm}^{-1}$; HRMS (ESI) m/z 414.1278 [414.1287 calcd for $\mathrm{C}_{21} \mathrm{H}_{18} \mathrm{~F}_{6} \mathrm{NO}$ $\left.(\mathrm{M}+\mathrm{H})^{+}\right]$.

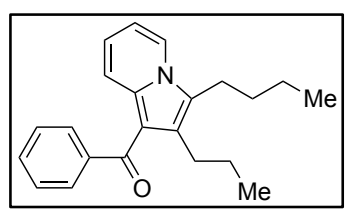

1-benzoyl-3-pentyl-2-propylindolizine (9g). Following the general procedure outlined above for the synthesis of compound 4, catalyst $5 \mathbf{c}(21 \mathrm{mg}, 0.024 \mathrm{mmol})$, 1-phenylethanol $(0.29 \mathrm{~mL})$ and acetophenone $(1.93 \mathrm{~mL})$ were added to 2-(4nonynyloxy)pyridine (8g, $105 \mathrm{mg}, 0.48 \mathrm{mmol})$. After 18 hours, the reaction was worked up and purified by column chromatography $\left(\mathrm{SiO}_{2}, 9: 1\right.$ hexanes/ethyl acetate) to afford $63 \mathrm{mg}$ (41\% yield) of $9 \mathrm{~g}$ as a thick yellow oil. ${ }^{1} \mathrm{H}$ NMR (400 MHz, $\left.C D C l_{3}\right): \delta 7.83(\mathrm{~d}, J=6.9 \mathrm{~Hz}, 1 \mathrm{H}), 7.68(\mathrm{~d}, J=7.1 \mathrm{~Hz}, 2 \mathrm{H}), 7.50$ $(\mathrm{t}, J=7.4 \mathrm{~Hz}, 1 \mathrm{H}), 7.42(\mathrm{t}, J=7.4 \mathrm{~Hz}, 2 \mathrm{H}), 7.16(\mathrm{~d}, J=9.0 \mathrm{~Hz}, 1 \mathrm{H}), 6.78(\mathrm{t}, J=7.6 \mathrm{~Hz}, 1 \mathrm{H}), 6.66(\mathrm{t}, J$ $=6.7 \mathrm{~Hz}, 1 \mathrm{H}), 2.86(\mathrm{t}, J=7.7 \mathrm{~Hz}, 2 \mathrm{H}), 2.74(\mathrm{t}, J=7.8 \mathrm{~Hz}, 2 \mathrm{H}), 1.39-1.60(\mathrm{~m}, 6 \mathrm{H}), 0.97(\mathrm{t}, J=7.2 \mathrm{~Hz}$, $3 \mathrm{H}), 0.85(\mathrm{t}, J=7.3 \mathrm{~Hz}, 3 \mathrm{H}) ;{ }^{13} \mathrm{C} \mathrm{NMR}\left(100 \mathrm{MHz}, \mathrm{CDCl}_{3}\right): \delta 192.2,142.2,136.1,130.9,129.6,128.8$, 128.2, 124.4, 122.5, 120.7, 119.3, 111.9, 111.6, 29.6, 27.5, 25.0, 23.4, 22.7, 14.3, 13.9; IR (neat): 3059, 2956, 2869, 1612, 1496, 1389, $1241 \mathrm{~cm}^{-1}$; HRMS (ESI) m/z 320.2013 [320.2009 calcd for $22{ }_{3} \mathrm{H}_{26} \mathrm{NO}$ $\left.(\mathrm{M}+\mathrm{H})^{+}\right]$.

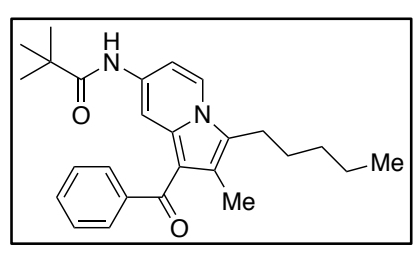

N-(1-benzoyl-2-methyl-3-pentylindolizin-7-yl)-2,2-dimethylpropanamide (11c). Following the general procedure outlined above for the synthesis of compound 4, catalyst 5c (15 mg, $0.017 \mathrm{mmol})$, 1-phenylethanol $(0.20 \mathrm{~mL})$ and acetophenone $(1.3 \mathrm{~mL})$ were added to 4-(2,2-dimethylpropanamide)-2(2-octynyloxy)pyridine (10c, $100 \mathrm{mg}, 0.33 \mathrm{mmol})$. After 18 hours, the reaction was worked up and 
S18 Supporting Information. Rossler, Hartgerink, Zerull, Anderson et. al.

purified by column chromatography ( $\mathrm{SiO}_{2}, 3: 1$ hexanes/ethyl acetate) to afford $87 \mathrm{mg}$ (65\% yield) of 11c as a yellow powder. mp: $175-179{ }^{\circ} \mathrm{C} ;{ }^{1} \mathrm{H}$ NMR $\left(400 \mathrm{MHz}, C D C l_{3}\right): \delta 8.92(\mathrm{~s}, 1 \mathrm{H}), 7.64(\mathrm{~d}, J=7.1$ $\mathrm{Hz}, 2 \mathrm{H}), 7.49$ (t, $J=7.4 \mathrm{~Hz}, 1 \mathrm{H}), 7.41$ ( app t, $J=7.8 \mathrm{~Hz}, 3 \mathrm{H}), 7.25(\mathrm{~s}, 1 \mathrm{H}), 6.56$ (dd, $J=2.0,9.5 \mathrm{~Hz}$, $1 \mathrm{H}), 2.84(\mathrm{t}, J=7.5 \mathrm{~Hz}, 2 \mathrm{H}), 2.16(\mathrm{~s}, 3 \mathrm{H}), 1.52-1.63(\mathrm{~m}, 4 \mathrm{H}), 1.32(\mathrm{~s}, 11 \mathrm{H}), 0.87(\operatorname{app~s}, 3 \mathrm{H}) ;{ }^{13} \mathrm{C}$ NMR $\left(100 \mathrm{MHz}_{\mathrm{CDCl}}\right): \delta 192.0,176.8,142.2,133.5,130.8,128.7,128.2,125.7,125.4,124.6,119.2,116.4$, 114.2, 112.6, 39.6, 31.4, 27.6, 26.8, 23.4, 22.5, 14.0, 12.0; IR (neat): 3281, 3088, 2951, 2924, 2856, 1670, 1589, $1490 \mathrm{~cm}^{-1}$; HRMS (ESI) m/z 405.2523 [405.2537 calcd for $\mathrm{C}_{26} \mathrm{H}_{33} \mathrm{~N}_{2} \mathrm{O}_{2}(\mathrm{M}+\mathrm{H})^{+}$].

N-(1-(3-fluorobenzoyl)-2-methyl-3-pentylindolizin-7-yl)-2,2-dimethylpropanamide

(11cc).

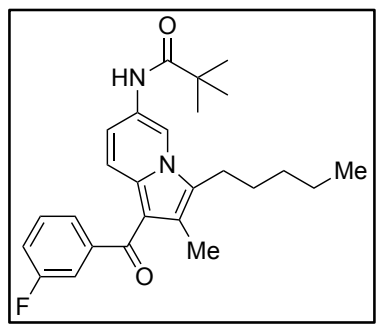

Following the general procedure outlined above for the synthesis of compound 4, catalyst 5c (15 mg, $0.017 \mathrm{mmol})$, 1-phenylethanol $(0.21 \mathrm{~mL})$ and 3 'fluoroacetophenone (1.4 mL) were added to 4-(2,2-dimethylpropanamide)-2-(2octynyloxy)pyridine (10c, $105 \mathrm{mg}, 0.34 \mathrm{mmol})$. After 18 hours, the reaction was worked up and purified by column chromatography $\left(\mathrm{SiO}_{2}, 85: 15\right.$ hexanes/ethyl acetate) to afford $81 \mathrm{mg}$ (56\% yield) of $11 \mathrm{cc}$ as a yellow powder. mp: $185-188{ }^{\circ} \mathrm{C} ;{ }^{1} \mathrm{H} \mathrm{NMR}(400 \mathrm{MHz}$, $\left.C D l_{3}\right): \delta 8.96(\mathrm{~s}, 1 \mathrm{H}), 7.49(\mathrm{~d}, J=9.3 \mathrm{~Hz}, 1 \mathrm{H}), 7.31-7.44(\mathrm{~m}, 3 \mathrm{H}), 7.25(\mathrm{~s}, 1 \mathrm{H}), 7.14-7.23(\mathrm{~m}, 2 \mathrm{H})$, $6.59(\mathrm{~d}, J=79.5 \mathrm{~Hz}, 1 \mathrm{H}), 2.84(\mathrm{t}, J=7.1 \mathrm{~Hz}, 2 \mathrm{H}), 2.16(\mathrm{~s}, 3 \mathrm{H}), 1.53-1.60(\mathrm{~m}, 4 \mathrm{H}), 1.33(\mathrm{~s}, 9 \mathrm{H}), 1.23$ (app s, 2H), 0.87 (app s, 3H); ${ }^{13} \mathrm{C}$ NMR (100 MHz, $\left.\mathrm{CDCl}_{3}\right): \delta 190.2,176.9,162.5$ (d, J=246 Hz), 144.4 $(\mathrm{d}, J=6.0 \mathrm{~Hz}), 133.7,129.8(\mathrm{~d}, J=30.8 \mathrm{~Hz}), 129.2,125.8,124.4,123.8,119.1,117.6$ (d, $J=21.2 \mathrm{~Hz})$, 117.0, 115.5 (d, $J=22.0 \mathrm{~Hz}), 114.3,112.1,39.6,31.4,27.6,26.8,23.4,22.5,14.0,12.0$; IR (neat): 3290, 3086, 2927, 2858, 1672, 1565, 1487, $1329 \mathrm{~cm}^{-1}$; HRMS (ESI) m/z 423.2434 [423.2442 calcd for $\left.\mathrm{C}_{26} \mathrm{H}_{32} \mathrm{FN}_{2} \mathrm{O}_{2}(\mathrm{M}+\mathrm{H})^{+}\right]$.

\section{2,2-dimethyl- $N$-(2-methyl-1-(3-nitrobenzoyl)-3-pentylindolizin-7-yl)-propanamide}

(11cce).

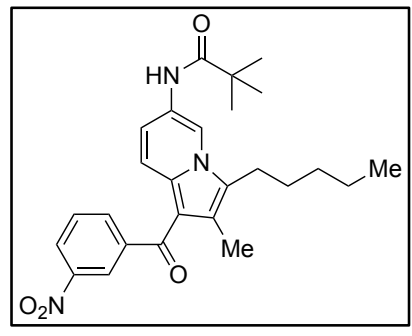

Following the general procedure outlined above for the synthesis of compound 4, catalyst 5c (15 mg, $0.017 \mathrm{mmol})$, 1-phenylethanol $(0.20 \mathrm{~mL})$ and 3'nitroacetophenone (2.8 g, $17.0 \mathrm{mmol})$ were added to 4-(2,2dimethylpropanamide)-2-(2-octynyloxy)pyridine (10c, $100 \mathrm{mg}, 0.33 \mathrm{mmol})$. After 18 hours, the reaction was worked up and purified by column chromatography ( $\mathrm{SiO}_{2}, 3: 1$ hexanes/ethyl acetate) to afford $96 \mathrm{mg}$ (65\% yield) of 11ccc as an orange powder. mp: $176-179{ }^{\circ} \mathrm{C} ;{ }^{1} \mathrm{H}$ NMR $\left(400 \mathrm{MHz}, C D C l_{3}\right): \delta 9.02(\mathrm{~s}, 1 \mathrm{H}), 8.47(\mathrm{~s}, 1 \mathrm{H}), 8.35(\mathrm{~d}, J=8.2 \mathrm{~Hz}$, $1 \mathrm{H}), 8.00(\mathrm{~d}, J=7.6 \mathrm{~Hz}, 1 \mathrm{H}), 7.53-7.68(\mathrm{~m}, 2 \mathrm{H}), 7.56(\mathrm{~s}, 1 \mathrm{H}), 6.66(\mathrm{~d}, J=9.4 \mathrm{~Hz}, 1 \mathrm{H}), 2.86(\mathrm{t}, J=7.3$ 
S19 Supporting Information. Rossler, Hartgerink, Zerull, Anderson et. al.

$\mathrm{Hz}, 2 \mathrm{H}), 2.11(\mathrm{~s}, 3 \mathrm{H}), 1.51-1.63(\mathrm{~m}, 2 \mathrm{H}), 1.34(\mathrm{~s}, 13 \mathrm{H}), 0.80-0.92(\mathrm{~m}, 3 \mathrm{H}) ;{ }^{13} \mathrm{C} \mathrm{NMR}\left(100 \mathrm{MHz}, \mathrm{CDCl}_{3}\right)$ : $\delta 188.6,176.9,148.0,143.8,134.5,134.0,129.5,126.5,126.1,125.2,124.2,123.8,119.0,117.5,114.5$, 111.6, 39.7, 31.4, 27.6, 26.8, 23.4, 22.5, 14.0, 12.3; IR (neat): 3293, 3088, 2924, 2858, 1670, 1529, 1488, $1345,1171 \mathrm{~cm}^{-1}$; HRMS (ESI) m/z 450.2384 [450.2387 calcd for $\mathrm{C}_{26} \mathrm{H}_{32} \mathrm{~N}_{3} \mathrm{O}_{4}(\mathrm{M}+\mathrm{H})^{+}$].

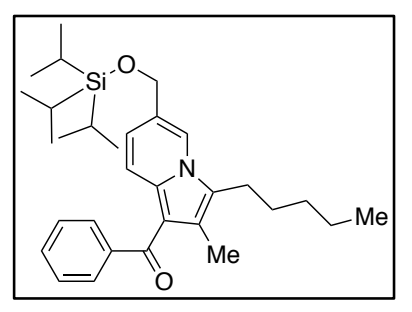

1-benzoyl-2-methyl-3-penty-6-(triisopropylsiloxymethyl)lindolizine (11d). Following the general procedure outlined above for the synthesis of compound 4, catalyst 5c (24 mg, $0.027 \mathrm{mmol})$, 1-phenylethanol $(0.31 \mathrm{~mL})$ and acetophenone $(2.0 \mathrm{~mL})$ were added to 4-(triisopropylsiloxymethyl)-2-(2octynyloxy)pyridine (10d, $199 \mathrm{mg}, 0.51 \mathrm{mmol})$. After 18 hours, the reaction was worked up and purified by column chromatography $\left(\mathrm{SiO}_{2}, 19: 1\right.$ hexanes/ethyl acetate) to afford 155 $\mathrm{mg}\left(62 \%\right.$ yield) of $11 \mathrm{~d}$ as a thick yellow oil. ${ }^{1} \mathrm{H} \mathrm{NMR}\left(400 \mathrm{MHz}, C D C l_{3}\right): \delta 7.96(\mathrm{~s}, 1 \mathrm{H}), 7.67(\mathrm{~d}, J=$ $7.2 \mathrm{~Hz}, 2 \mathrm{H}), 7.48$ (t, $J=7.1 \mathrm{~Hz}, 1 \mathrm{H}), 7.41(\mathrm{t}, J=7.3 \mathrm{~Hz}, 2 \mathrm{H}), 7.36(\mathrm{~d}, J=9.2 \mathrm{~Hz}, 2 \mathrm{H}), 6.70(\mathrm{~d}, J=9.2$ $\mathrm{Hz}, 1 \mathrm{H}), 4.81 \mathrm{~s}, 2 \mathrm{H}), 2.84(\mathrm{t}, J=7.4 \mathrm{~Hz}, 2 \mathrm{H}), 2.23$ (s, 3H), 1.52-1.59 (m, 2H), 1.30-1.41 (m, 4H), 1.14$1.27(\mathrm{~m}, 3 \mathrm{H}), 1.10(\mathrm{~d}, \mathrm{~J}=6.8 \mathrm{~Hz}, 18 \mathrm{H}), 0.89(\mathrm{t}, J=5.5 \mathrm{~Hz}, 3 \mathrm{H}) ;{ }^{13} \mathrm{C} \mathrm{NMR}\left(100 \mathrm{MHz}, \mathrm{CDCl}_{3}\right): \delta 192.0$, $142.4,135.5,130.7,128.7,128.1,126.1,124.8,124.1,120.2,119.1,118.8,112.2,62.8,51.7,27.1,23.7$, 22.6, 18.0, 14.0, 11.9; IR (neat): 2940, 2863, 1613, 1505, 1394, 1241, $1098 \mathrm{~cm}^{-1}$; HRMS (ESI) m/z 492.3285 [492.3292 calcd for $\left.\mathrm{C}_{31} \mathrm{H}_{46} \mathrm{NO}_{2} \mathrm{Si}(\mathrm{M}+\mathrm{H})^{+}\right]$.

\section{1-(3,5-bistrifluoromethylbenzoyl)-2-methyl-3-penty-6-(triisopropylsiloxymethyl)lindolizine}

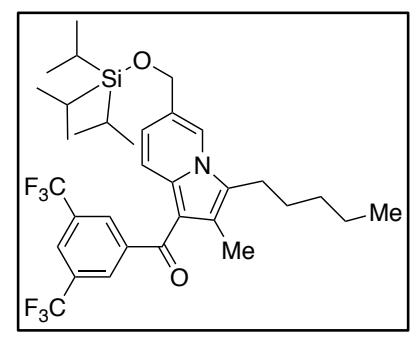

(11dd). Following the general procedure outlined above for the synthesis of compound 4, catalyst 5c $(28 \mathrm{mg}, 0.027 \mathrm{mmol})$, 1-phenylethanol $(0.32 \mathrm{~mL})$ and 3',5'-bistrifluoromethylacetophenone $(2.0 \mathrm{~mL})$ were added to 4(triisopropylsiloxymethyl)-2-(2-octynyloxy)pyridine (10d, $209 \mathrm{mg}, 0.54$ mmol). After 18 hours, the reaction was worked up and purified by column chromatography $\left(\mathrm{SiO}_{2}, 97: 3\right.$ hexanes/ethyl acetate) to afford $291 \mathrm{mg}$ (86\% yield) of 11dd as a yellow solid. mp: $85-87^{\circ} \mathrm{C} ;{ }^{1} \mathrm{H}$ NMR $\left(400 \mathrm{MHz}, C D C l_{3}\right): \delta 8.12(\mathrm{~s}, 2 \mathrm{H}), 8.03(\mathrm{~s}, 1 \mathrm{H}), 7.99(\mathrm{~s}, 1 \mathrm{H}), 7.55(\mathrm{~d}, J=$ $9.1 \mathrm{~Hz}, 1 \mathrm{H}), 6.88(\mathrm{~d}, J=9.2 \mathrm{~Hz}, 1 \mathrm{H}), 4.86(\mathrm{~s}, 2 \mathrm{H}), 2.85(\mathrm{t}, J=7.4 \mathrm{~Hz}, 2 \mathrm{H}), 2.12(\mathrm{~s}, 3 \mathrm{H}), 1.51-1.59$ (m, 2H), 1.29-1.39 (m, 4H), 1.15-1.27 (m, 3H), $1.11(\mathrm{~d}, J=6.9 \mathrm{~Hz}, 18 \mathrm{H}), 0.89(\mathrm{t}, J=4.8 \mathrm{~Hz}, 3 \mathrm{H}) ;{ }^{13} \mathrm{C}$ NMR (100 MHz, $\left.\mathrm{CDCl}_{3}\right): \delta 187.7,144.1,136.2,131.6(\mathrm{q}, J=34 \mathrm{~Hz}), 129.0$ (q, $\left.J=3.5 \mathrm{~Hz}\right), 127.3,125.7$, $124.5,123.6,123.1(\mathrm{q}, J=272 \mathrm{~Hz}), 121.9,119.6,118.5,111.1,62.7,31.7,27.0,23.7,22.6,18.0,14.0$, 
S20 Supporting Information. Rossler, Hartgerink, Zerull, Anderson et. al.

12.1, 12.0; IR (neat): 2942, 2866, 1627, 1504, 1367, 1278, $1138 \mathrm{~cm}^{-1}$; HRMS (ESI) m/z 628.3022 [628.3040 calcd for $\left.\mathrm{C}_{33} \mathrm{H}_{44} \mathrm{~F}_{6} \mathrm{NO}_{2} \mathrm{Si}(\mathrm{M}+\mathrm{H})^{+}\right]$.

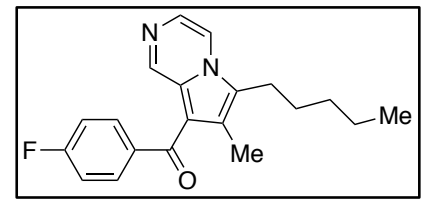

\section{8-(4-fluorobenzoyl)-7-methyl-6-pentylpyrrolo[1,2-a]pyrazine}

(11e).

Following the general procedure outlined above for the synthesis of compound 4, catalyst 5c (22 mg, $0.025 \mathrm{mmol})$, 1-phenylethanol $(0.30 \mathrm{~mL})$ and 4'-fluoroacetophenone (2.0 mL) were added to 2-(2-octynyloxy)pyrazine (10e, $100 \mathrm{mg}, 0.49 \mathrm{mmol})$. After 18 hours, the reaction was worked up and purified by column chromatography $\left(\mathrm{SiO}_{2}, 7: 3\right.$ hexanes/ethyl acetate) to afford $12 \mathrm{mg}$ (8\% yield) of $11 \mathrm{e}$ as a yellow oil. ${ }^{1} \mathrm{H}$ NMR $\left(400 \mathrm{MHz}, C D C l_{3}\right)$ : $\delta 8.60(\mathrm{~s}, 1 \mathrm{H}), 7.77(\mathrm{t}, J=6.3 \mathrm{~Hz}, 2 \mathrm{H}), 7.71(\mathrm{~s}, 2 \mathrm{H}), 7.14(\mathrm{t}, J=8.2 \mathrm{~Hz}, 2 \mathrm{H}), 2.87$ (t, $J=7.5 \mathrm{~Hz}, 2 \mathrm{H})$, $2.31(\mathrm{~s}, 3 \mathrm{H}), 1.55-1.64(\mathrm{~m}, 2 \mathrm{H}), 1.31-1.38(\mathrm{~m}, 4 \mathrm{H}), 0.86-0.92(\mathrm{~m}, 3 \mathrm{H}) ;{ }^{13} \mathrm{C} \mathrm{NMR}\left(100 \mathrm{MHz}, \mathrm{CDCl}_{3}\right): \delta$ 190.4, 178.6, 165.2 (d, $J=252 \mathrm{~Hz}), 144.4,137.1,131.6$ (d, $J=9.0 \mathrm{~Hz}), 129.2,129.0,126.4(\mathrm{~d}, J=244$ Hz), 115.5 (d, $J=21 \mathrm{~Hz}), 115.2,114.9,31.5,26.8,23.3,22.4,14.0,11.4$; IR (neat): 2954, 2926, 2857, 1629, 1597, 1495, 1384, 1241, $1151 \mathrm{~cm}^{-1}$; HRMS (ESI) m/z 325.1703 [325.1711 calcd for $\mathrm{C}_{20} \mathrm{H}_{22} \mathrm{FN}_{2} \mathrm{O}$ $\left.(\mathrm{M}+\mathrm{H})^{+}\right]$.

8-(3,5-bistrifluoromethylbenzoyl)-7-methyl-6-pentylpyrrolo[1,2-a]pyrazine (11ee). Following the

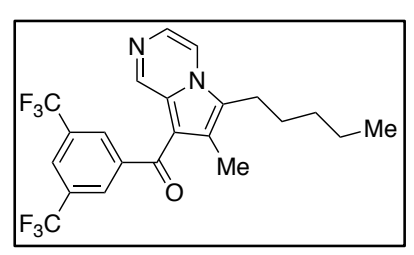
general procedure outlined above for the synthesis of compound 4 , catalyst $\mathbf{5 c}$ (22 mg, 0.025 mmol), 1-phenylethanol $(0.30 \mathrm{~mL})$ and 3'5'bistrifluoromethylacetophenone $(2.0 \mathrm{~mL})$ were added to 2-(2octynyloxy)pyrazine (10e, $100 \mathrm{mg}, 0.49 \mathrm{mmol})$. After 18 hours, the reaction was worked up and purified by column chromatography $\left(\mathrm{SiO}_{2}, 7: 3\right.$ hexanes/ethyl acetate) to afford 59 $\mathrm{mg}\left(27 \%\right.$ yield) of $11 \mathrm{ee}$ as an orange solid. mp: $140-142{ }^{\circ} \mathrm{C} ;{ }^{1} \mathrm{H}$ NMR (400 MHz, $\left.C D C l 3\right): \delta 8.80$ (s, $1 \mathrm{H}), 8.18(\mathrm{~s}, 2 \mathrm{H}), 8.07(\mathrm{~s}, 1 \mathrm{H}), 7.86-7.75(\mathrm{~m}, 2 \mathrm{H}), 2.90(\mathrm{t}, J=7.7 \mathrm{~Hz}, 2 \mathrm{H}), 2.22(\mathrm{~s}, 3 \mathrm{H}), 1.56-1.69$ (m, 2H), 1.33-1.40 (m, 4H), $0.91(\mathrm{t}, J=6.9 \mathrm{~Hz}, 3 \mathrm{H}) ;{ }^{13} \mathrm{C} \mathrm{NMR}\left(100 \mathrm{MHz}, \mathrm{CDCl}_{3}\right): \delta 188.4,144.3,142.8$, $132.2(\mathrm{q}, J=34 \mathrm{~Hz}), 130.3,129.8,129.3$ (q, $J=3.9 \mathrm{~Hz}), 127.4,126.0,125.2$ (heptet, $J=3.8 \mathrm{~Hz}), 123.1$ (q, $J=273 \mathrm{~Hz}), 115.3,114.1,31.7,26.9,23.4,22.6,14.1,12.0$; IR (neat): 2929, 2860, 1634, 1608, 1495, $1174 \mathrm{~cm}^{-1}$; HRMS (ESI) m/z 443.1557 [443.1553 calcd for $\left.\mathrm{C}_{22} \mathrm{H}_{21} \mathrm{~F}_{6} \mathrm{~N}_{2} \mathrm{O}(\mathrm{M}+\mathrm{H})^{+}\right]$. 
S21 Supporting Information. Rossler, Hartgerink, Zerull, Anderson et. al.

1-benzoyl-2,8-dimethyl-3-pentylindolizine (13). Following the general procedure outlined above for

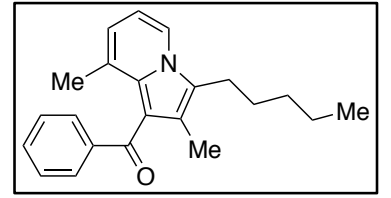
the synthesis of compound 4, catalyst $5 \mathrm{c}$ (25 mg, $0.029 \mathrm{mmol})$, 1-phenylethanol $(0.31 \mathrm{~mL})$ and acetophenone $(2.0 \mathrm{~mL})$ were added to 3-methyl-2-(2octynoxy)pyridine $(\mathbf{1 2}, 116 \mathrm{mg}, 0.53 \mathrm{mmol})$. After 18 hours, the reaction was worked up and purified by column chromatography $\left(\mathrm{SiO}_{2}, 97: 3\right.$ hexanes/ethyl acetate) to afford $78 \mathrm{mg}$ (46\% yield) of 13 as a thick yellow oil. ${ }^{1} \mathrm{H}$ NMR (400 MHz, $\left.C D C l 3\right): \delta 7.82(\mathrm{~d}, J=7.6 \mathrm{~Hz}, 2 \mathrm{H}), 7.73$ (d, $J=6.0 \mathrm{~Hz}, 1 \mathrm{H}), 7.52(\mathrm{t}, J=7.0 \mathrm{~Hz}, 1 \mathrm{H}), 7.41(\mathrm{t}, J=7.3 \mathrm{~Hz}, 2 \mathrm{H}), 6.61(\mathrm{t}, J=6.8 \mathrm{~Hz}, 1 \mathrm{H}), 2.83(\mathrm{t}, J=$ $7.3 \mathrm{~Hz}, 2 \mathrm{H}), 2.16(\mathrm{~s}, 3 \mathrm{H}), 1.98(\mathrm{~s}, 3 \mathrm{H}), 1.51-1.72(\mathrm{~m}, 2 \mathrm{H}), 1.33(\operatorname{app~s}, 4 \mathrm{H}), 0.88(\mathrm{t}, J=5.3 \mathrm{~Hz}, 3 \mathrm{H}) ;{ }^{13} \mathrm{C}$ NMR (100 MHz, $\left.\mathrm{CDCl}_{3}\right): \delta 194.3,141.3,132.8,132.1,130.0,128.8,128.2,122.9,122.1,120.8,120.1$, 113.6, 111.3, 31.6, 27.1, 23.8, 22.5, 21.0, 14.0, 11.3; IR (neat): 2923, 2856, 1633, 1492, 1389, $1231 \mathrm{~cm}^{-}$ 1; HRMS (ESI) m/z 320.2003 [320.2009 calcd for $\mathrm{C}_{22} \mathrm{H}_{26} \mathrm{NO}(\mathrm{M}+\mathrm{H})^{+}$].

2,8-dimethyl-3-pentyl-1-(3,5-bistrifluoromethylbenzoyl)indolizine (13a). Following the general

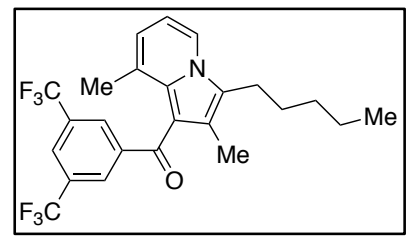
procedure outlined above for the synthesis of compound 4, catalyst $5 \mathrm{c}(24 \mathrm{mg}$, $0.026 \mathrm{mmol})$, 1-phenylethanol $(0.32 \mathrm{~mL})$ and 3',5'bistrifluoromethylacetophenone $(2.0 \mathrm{~mL})$ were added to 3-methyl-2-(2octynoxy)pyridine $(\mathbf{1 2}, 114 \mathrm{mg}, 0.53 \mathrm{mmol})$. After 18 hours, the reaction was worked up and purified by column chromatography ( $\mathrm{SiO}_{2}, 98: 2$ hexanes/ethyl acetate) to afford $110 \mathrm{mg}$ (46\% yield) of 13a as a deep orange solid. mp: 86-87 ${ }^{\circ} \mathrm{C} ;{ }^{1} \mathrm{H}$ NMR (400 MHz, $\left.C D C l 3\right): \delta 8.30(\mathrm{~s}, 2 \mathrm{H})$, $8.04(\mathrm{~s}, 1 \mathrm{H}), 7.80(\mathrm{~d}, J=6.4 \mathrm{~Hz}, 1 \mathrm{H}), 6.81(\mathrm{~d}, J=6.0 \mathrm{~Hz}, 1 \mathrm{H}), 6.71(\mathrm{t}, J=6.6 \mathrm{~Hz}, 1 \mathrm{H}), 2.86(\mathrm{t}, J=7.2$ $\mathrm{Hz}, 2 \mathrm{H}), 2.26(\mathrm{~s}, 3 \mathrm{H}), 1.91(\mathrm{~s}, 3 \mathrm{H}), 1.51-1.60(\mathrm{~m}, 3 \mathrm{H}), 1.33(\operatorname{app~s}, 4 \mathrm{H}), 0.88(\operatorname{app~s}, 3 \mathrm{H}) ;{ }^{13} \mathrm{C}$ NMR $(100$ $\left.\mathrm{MHz}, \mathrm{CDCl}_{3}\right): \delta 189.4,142.8,134.1,131.8(\mathrm{q}, J=34 \mathrm{~Hz}), 130.1,130.0,128.9,125.0$ (heptet, $J=3.9$ Hz), 123.9, 123.1 (q, $J=272$ Hz), 122.8, 121.8, 120.6, 112.3, 31.5, 26.9, 23.7, 22.5, 21.2, 14.0, 12.0; IR (neat): 2927, 2861, 1639, 1489, 1365, 1278, $1136 \mathrm{~cm}^{-1}$; HRMS (ESI) m/z 456.1758 [456.1757 calcd for $\left.\mathrm{C}_{24} \mathrm{H}_{24} \mathrm{~F}_{6} \mathrm{NO}(\mathrm{M}+\mathrm{H})^{+}\right]$. 
S22 Supporting Information. Rossler, Hartgerink, Zerull, Anderson et. al.

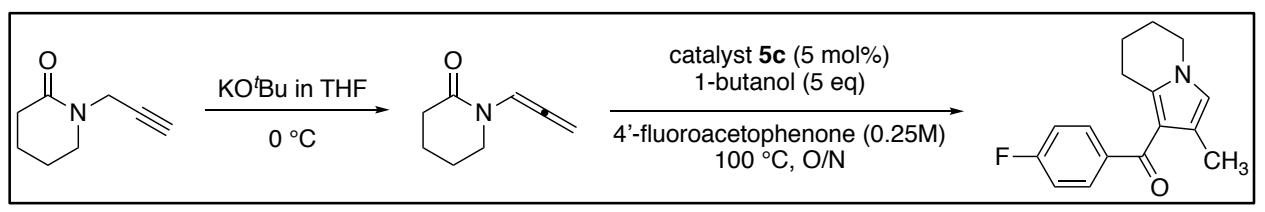

To a solution of $N$-propargylvalerolactam $(223 \mathrm{mg}, 1.63 \mathrm{mmol})$ in THF $(5 \mathrm{~mL})$ at $0{ }^{\circ} \mathrm{C}$ was added potassium tert-butoxide (1.0 M in THF, $0.49 \mathrm{~mL}, 0.49 \mathrm{mmol})$. After 90 minutes, $\mathrm{Et}_{2} \mathrm{O}(20 \mathrm{~mL})$ was added and the mixture was filtered through celite. After removing the excess solvent in vacuo, the residual was used directly in the $\mathrm{Au}(\mathrm{I})$-catalyzed transformation.

Following the general procedure outlined above for the synthesis of compound 4, catalyst $5 \mathbf{c}$ ( 29 $\mathrm{mg}, 0.033 \mathrm{mmol})$, 1-butanol $(0.30 \mathrm{~mL})$ and 4'-fluoroacetophenone $(2.62 \mathrm{~mL})$ were added to $N$ (1,2-propadienyl)valerolactam $(\mathbf{1 5}, 90 \mathrm{mg}, 0.66 \mathrm{mmol})$. After 18 hours, the reaction was worked up and purified by column chromatography $\left(\mathrm{SiO}_{2}, 85: 15\right.$ hexanes/ethyl acetate) to afford $7 \mathrm{mg}$ ( $8 \%$ yield) of $\mathbf{1 6}$ as a clear oil that decomposes both during purification and upon standing. ${ }^{1} \mathrm{H}$ NMR (400 $\left.\mathrm{MHz}, C D C l_{3}\right): \delta 7.74-7.61(\mathrm{~m}, 2 \mathrm{H}), 7.09(\mathrm{t}, J=8.7 \mathrm{~Hz}, 2 \mathrm{H}), 6.30(\mathrm{~s}, 1 \mathrm{H}), 3.88(\mathrm{t}, J=6.8 \mathrm{~Hz}, 2 \mathrm{H}), 2.70$ (t, $J=6.8 \mathrm{~Hz}, 2 \mathrm{H}), 1.90-1.97$ (m, 5H), 1.68-1.79 (m, 2H); ${ }^{13} \mathrm{C}$ NMR (400 MHz, CDCl3): 192.2, 136.5, $131.0(\mathrm{~d}, J=8.6 \mathrm{~Hz}), 120.2,119.2,119.0,115.0(\mathrm{~d}, J=21.6 \mathrm{~Hz}), 53.4,45.3,30.9,24.3,22.9,20.4$, 12.2; IR (neat): 3280, 2942, 2850, 1722, 1613, 1471, $1242 \mathrm{~cm}^{-1}$; HRMS (ESI) m/z 258.1262 [258.1289 calcd for $\left.\mathrm{C}_{16} \mathrm{H}_{17} \mathrm{FNO}(\mathrm{M}+\mathrm{H})^{+}\right]$. 
S23 Supporting Information. Rossler, Hartgerink, Zerull, Anderson et. al.

\section{B. Optimization Studies}
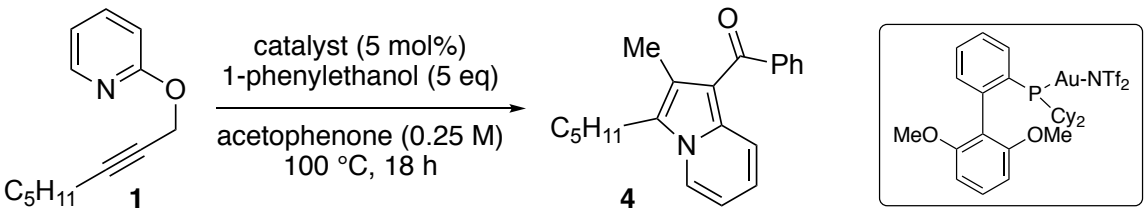

entry

modification or additive

Compound 4

(\% yield)

\begin{tabular}{ccc}
\hline 1 & - & 54 \\
2 & $\mathrm{MgSO}_{4}$ & 54 \\
3 & $\mathrm{Na}_{2} \mathrm{SO}_{4}$ & 55 \\
4 & $\mathrm{CaSO}_{4}$ & 53 \\
5 & molecular sieves & 58 \\
6 & $\mathrm{~K}_{2} \mathrm{CO}_{3}$ & 53 \\
7 & pyridine & 38 \\
8 & morpholine & 16 \\
9 & $\mathrm{TsOH}$ & 42 \\
10 & $160{ }^{\circ} \mathrm{C}$ & 42 \\
\hline
\end{tabular}


S24 Supporting Information. Rossler, Hartgerink, Zerull, Anderson et. al.

\section{Experimental Details for the X-ray Crystal Structure of Compound 4}

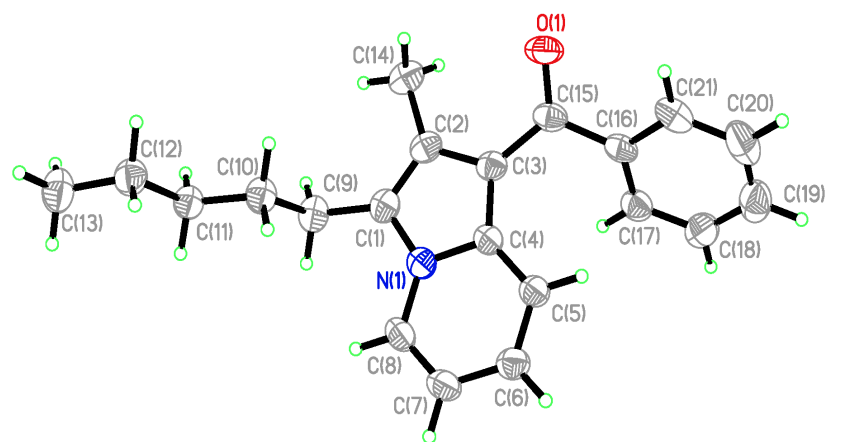

Figure 1. ORTEP of Indolizine 4 Solved at $0.72 \AA$ Resolution

A colorless chunk-shaped crystal with dimensions $0.27 \times 0.27 \times 0.15 \mathrm{~mm}^{3}$ was mounted on a nylon loop with paratone oil. Data were collected using a Bruker APEX-II CCD diffractometer equipped with an Oxford Cryosystems low-temperature device, operating at $T=173(2) \mathrm{K}$.

Data were measured using $\phi$ and $\omega$ scans of $1.00^{\circ}$ per frame for $20.00 \mathrm{~s}$ using $\mathrm{CuK}$ radiation (sealed tube, $40 \mathrm{kV}, 30 \mathrm{~mA}$ ). The total number of runs and images was based on the strategy calculation from the program COSMO. ${ }^{1}$ The actually achieved resolution was $\Theta=71.969$. Cell parameters were retrieved using the SAINT software ${ }^{2}$ and refined using SAINT on 8646 reflections, $76 \%$ of the observed reflections. Data reduction was performed using the SAINT software which corrects for Lorentz polarization. The final completeness is 98.80 out to 71.969 in $\Theta$. The absorption coefficient $\mu$ of this material is 0.563 at this wavelength $(\lambda=1.54178)$ and the minimum and maximum transmissions are 0.6895 and 0.7535 .

The structure was solved in the space group P $2{ }_{1} 2{ }_{1} 2_{1}$ (\# 19) by Direct Methods using the ShelXS structure solution program. ${ }^{3}$ The structure was refined by Least Squares using version 2014/6 of XL incorporated in Olex2. ${ }^{4}$ All non-hydrogen atoms were refined anisotropically. Hydrogen atom positions were calculated geometrically and refined using the riding model. There is a single molecule in the asymmetric unit, which is represented by the reported sum formula. In other words: $Z$ is 4 and $Z^{\prime}$ is 1 . The Flack parameter was refined to 0.05(11). Determination of absolute structure using Bayesian statistics on Bijvoet differences using the Olex2 results in 0.07(11). Note: The Flack parameter is used to determine chirality of the crystal studied, the value should be near 0 , a value of 1 means that the stereochemistry is wrong and the model should be inverted. A value of 0.5 means that the crystal consists of a racemic mixture of the two enantiomers.

\footnotetext{
${ }^{1}$ COSMO-V1.61, Software for the CCD Detector Systems for Determining Data Collection Parameters. Bruker Analytical X-ray Systems, Madison, WI (2009).

2 SAINT-8.34A-2013. Software for the Integration of CCD Detector System. Bruker Analytical X-ray Systems, Madison, WI (2013).

3 Sheldrick, G. M. “A short history of ShelX” Acta Cryst., 2008, A64, 339-341.

4 Dolomanov, O. V.; Bourhis, L. J.; Gildea, R. J.; Howard, J. A. K.; Puschmann, H. “Olex2: A complete structure solution, refinement and analysis program" J. Appl. Cryst., 2009, 42, 339-341.
} 
S25 Supporting Information. Rossler, Hartgerink, Zerull, Anderson et. al.
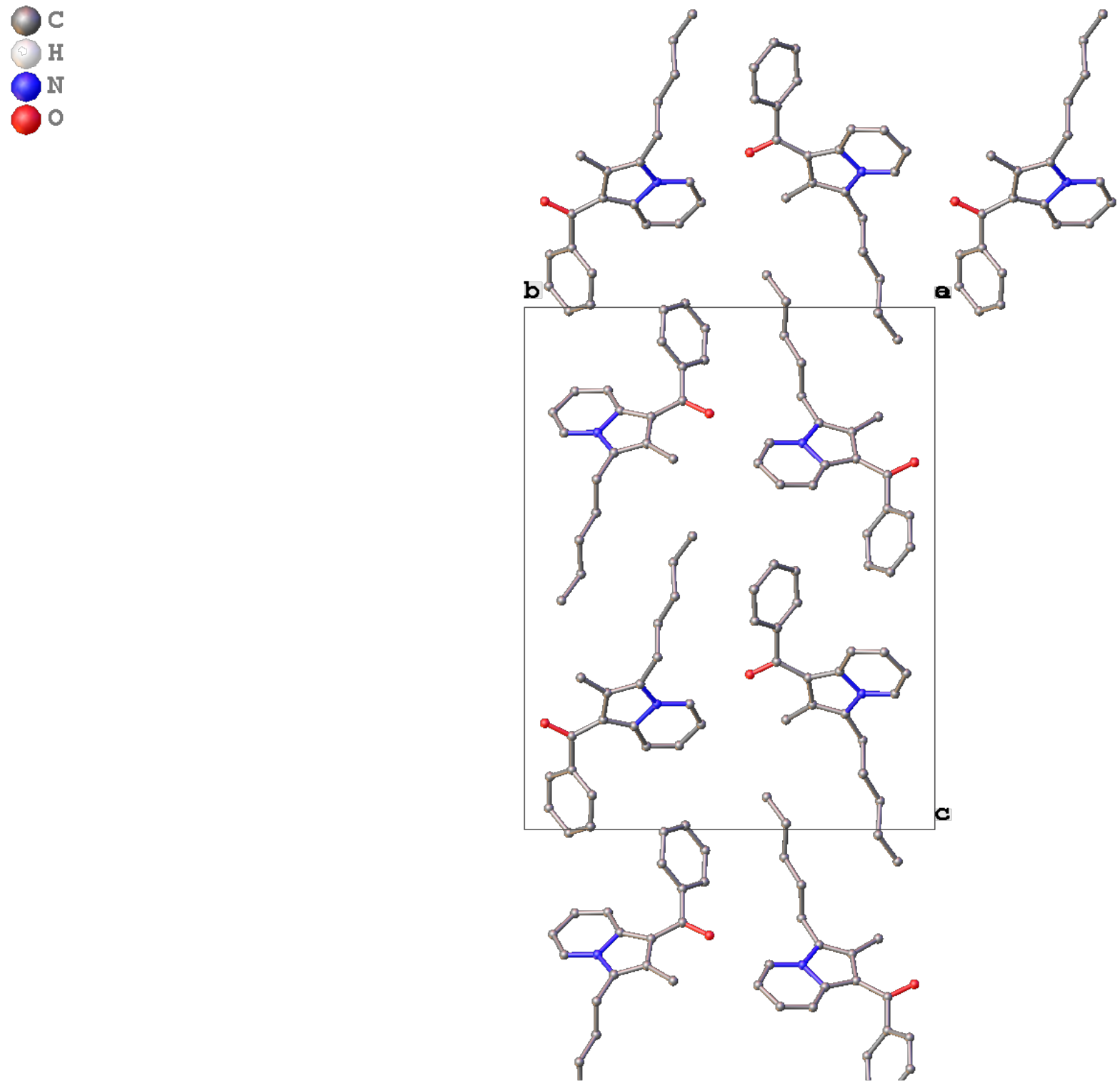

Figure 2. Solid state packing diagram for compound 4. 
S26 Supporting Information. Rossler, Hartgerink, Zerull, Anderson et. al.

D. ${ }^{1} \mathrm{H},{ }^{13} \mathrm{C}$ NMR, and HRMS of Isotopically Labelled Indolizine 4/4'

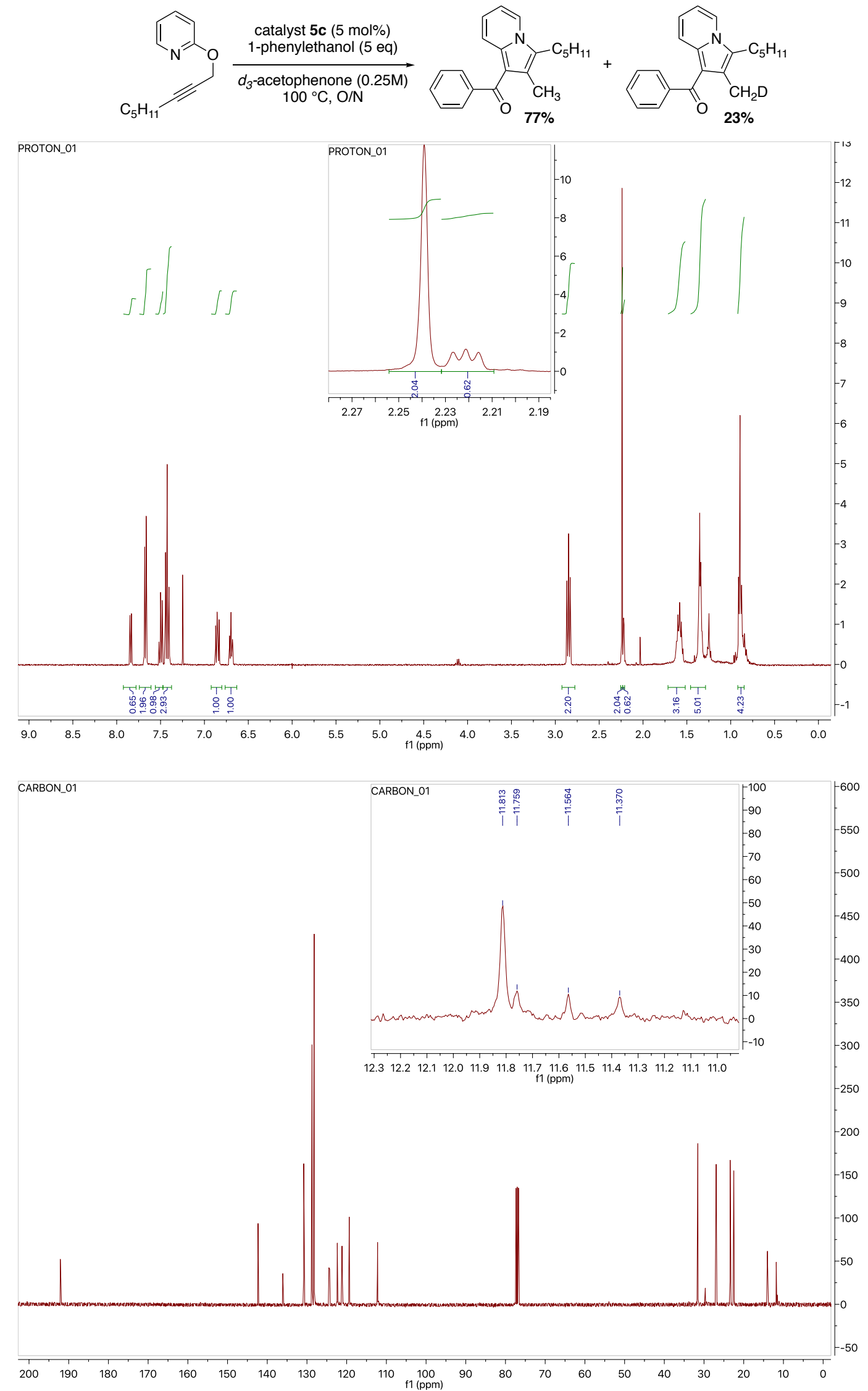


S27 Supporting Information. Rossler, Hartgerink, Zerull, Anderson et. al.

IIJ Mass Spectrometry \& Proteomics Facility

Mass Spectrum SmartFormula Report

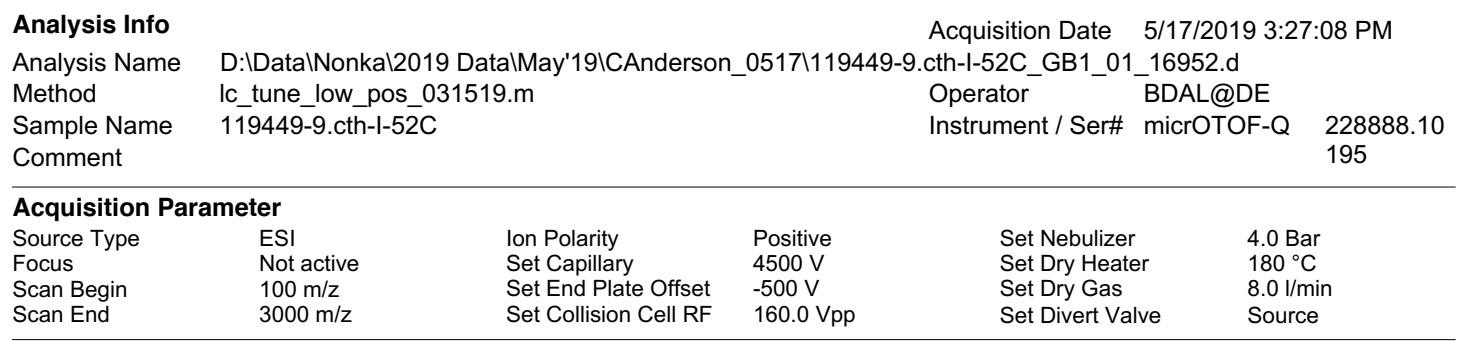

$\begin{array}{lllll}\text { Scan End } & 3000 \mathrm{~m} / \mathrm{z} & \text { Set Collision Cell RF } & 160.0 \mathrm{Vpp} & \text { Set Divert Valve }\end{array}$
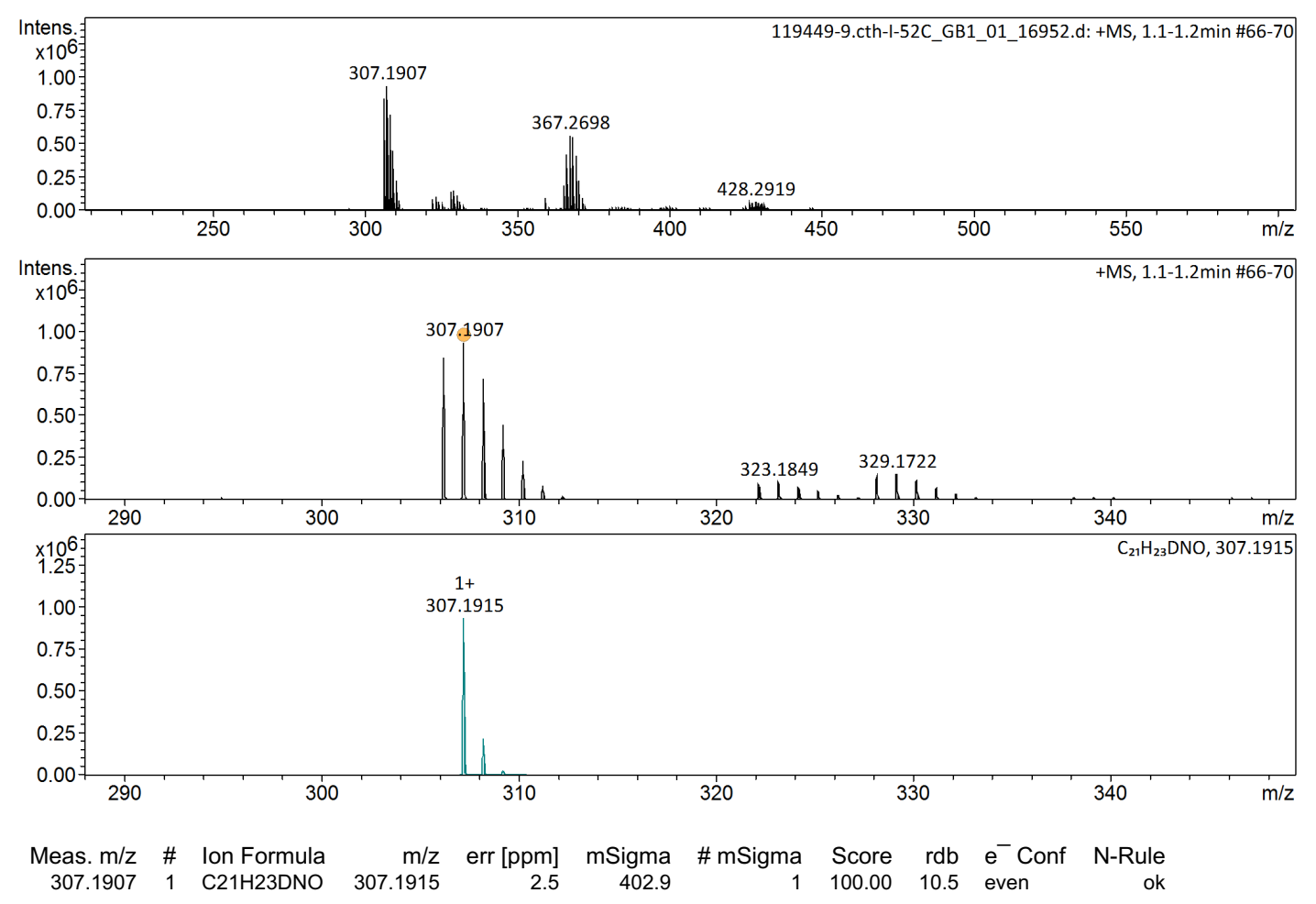

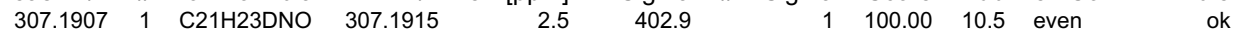

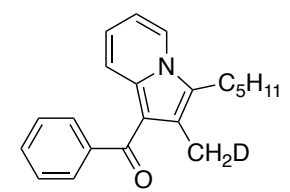

Chemical Formula: $\mathrm{C}_{21} \mathrm{H}_{22} \mathrm{DNO}$

Exact Mass $[\mathrm{M}+\mathrm{H}]^{+}: 307.1915$

Bruker Compass DataAnalysis 4.2

printed: $\quad$ 5/17/2019 4:12:06 PM

Page 1 of 1

This material is based upon work supported by the National Science Foundation under CHE-0741793 
S28 Supporting Information. Rossler, Hartgerink, Zerull, Anderson et. al.

\section{E. ${ }^{1} \mathrm{H}$ and ${ }^{13} \mathrm{C}$ NMR Spectra of New Compounds}

\section{Compound 8a}

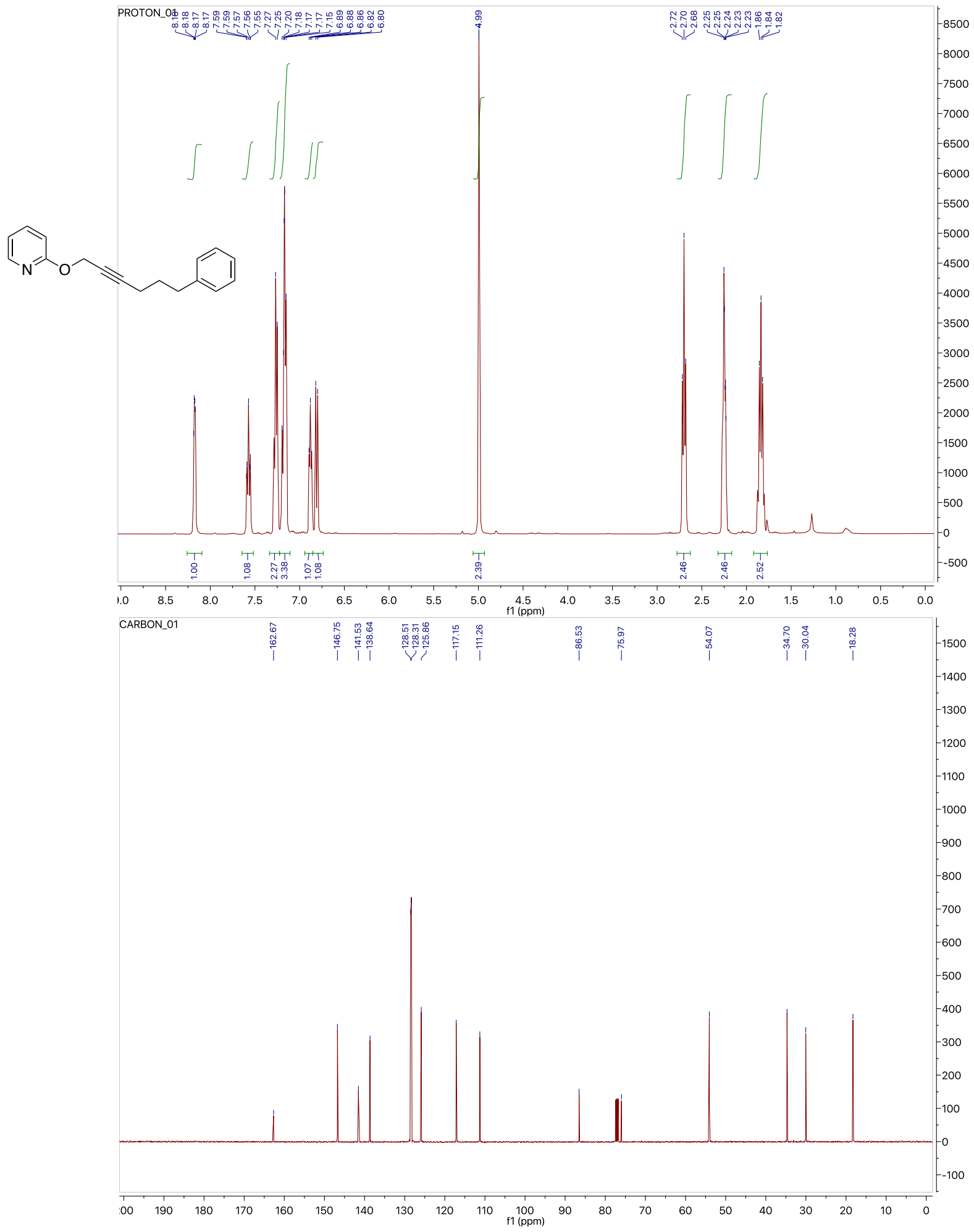


S29 Supporting Information. Rossler, Hartgerink, Zerull, Anderson et. al.

\section{Compound 8g}
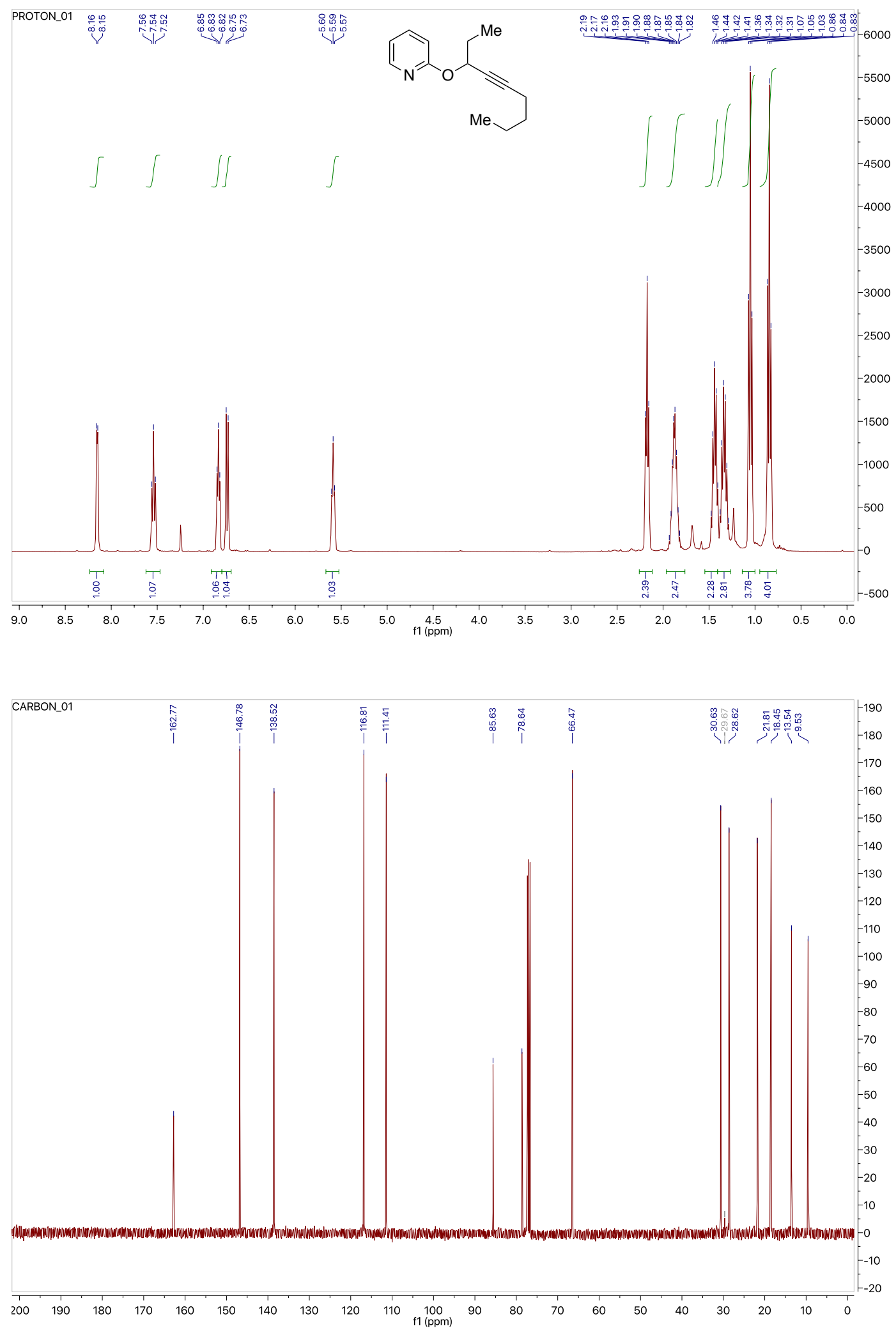
S30 Supporting Information. Rossler, Hartgerink, Zerull, Anderson et. al.

\section{Compound 10a}
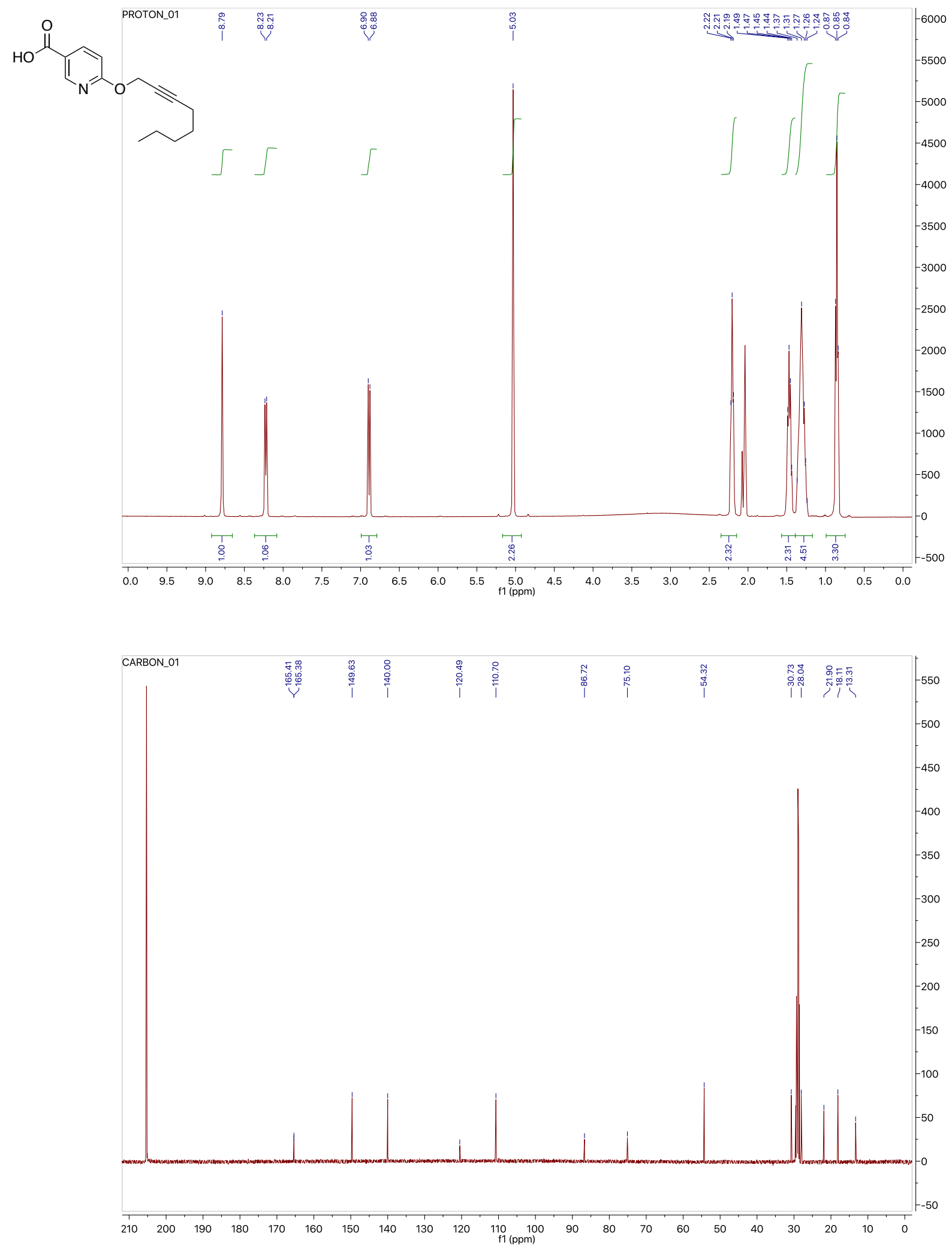
S31 Supporting Information. Rossler, Hartgerink, Zerull, Anderson et. al.

\section{Compound 10c}
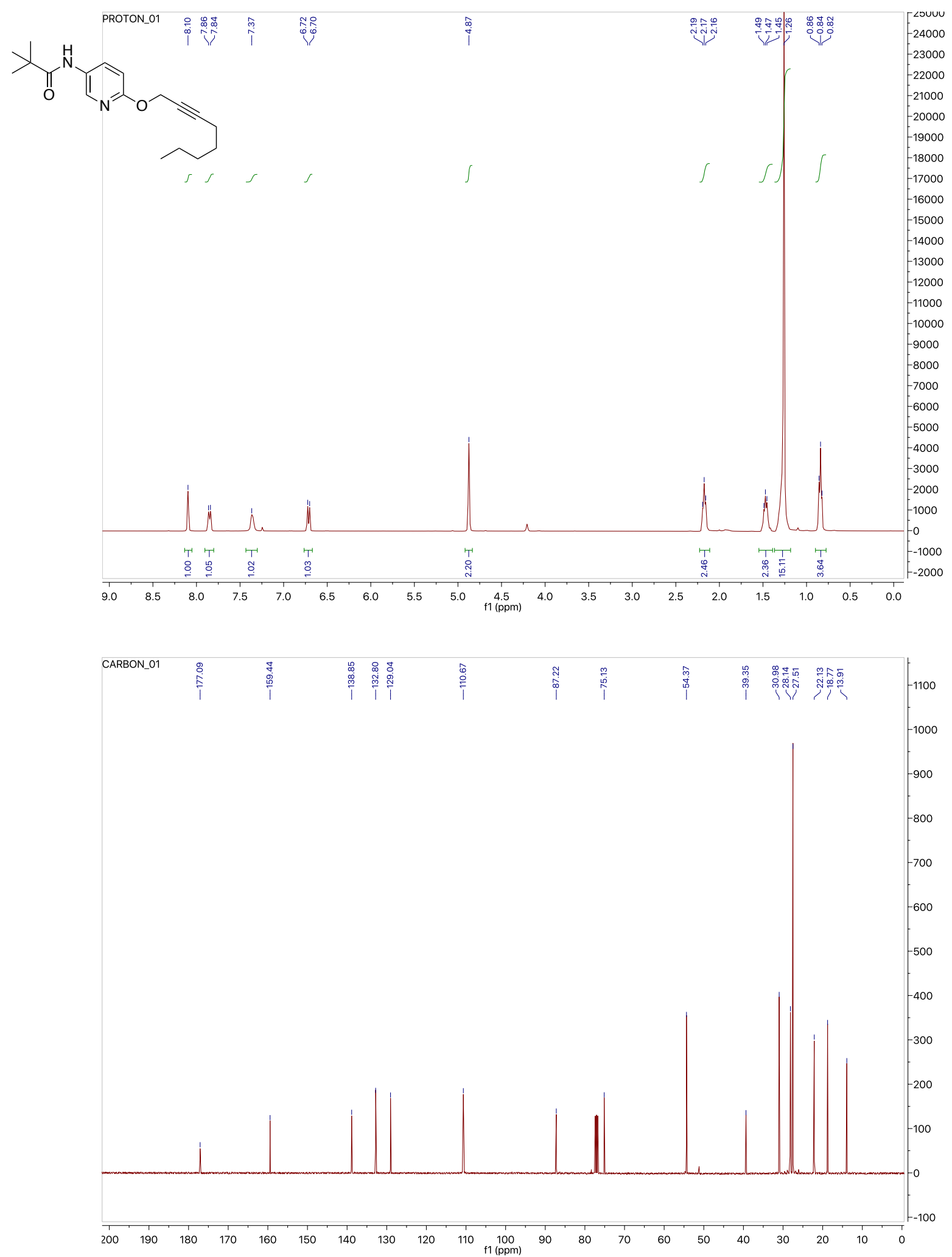
S32 Supporting Information. Rossler, Hartgerink, Zerull, Anderson et. al.

\section{Compound 22}
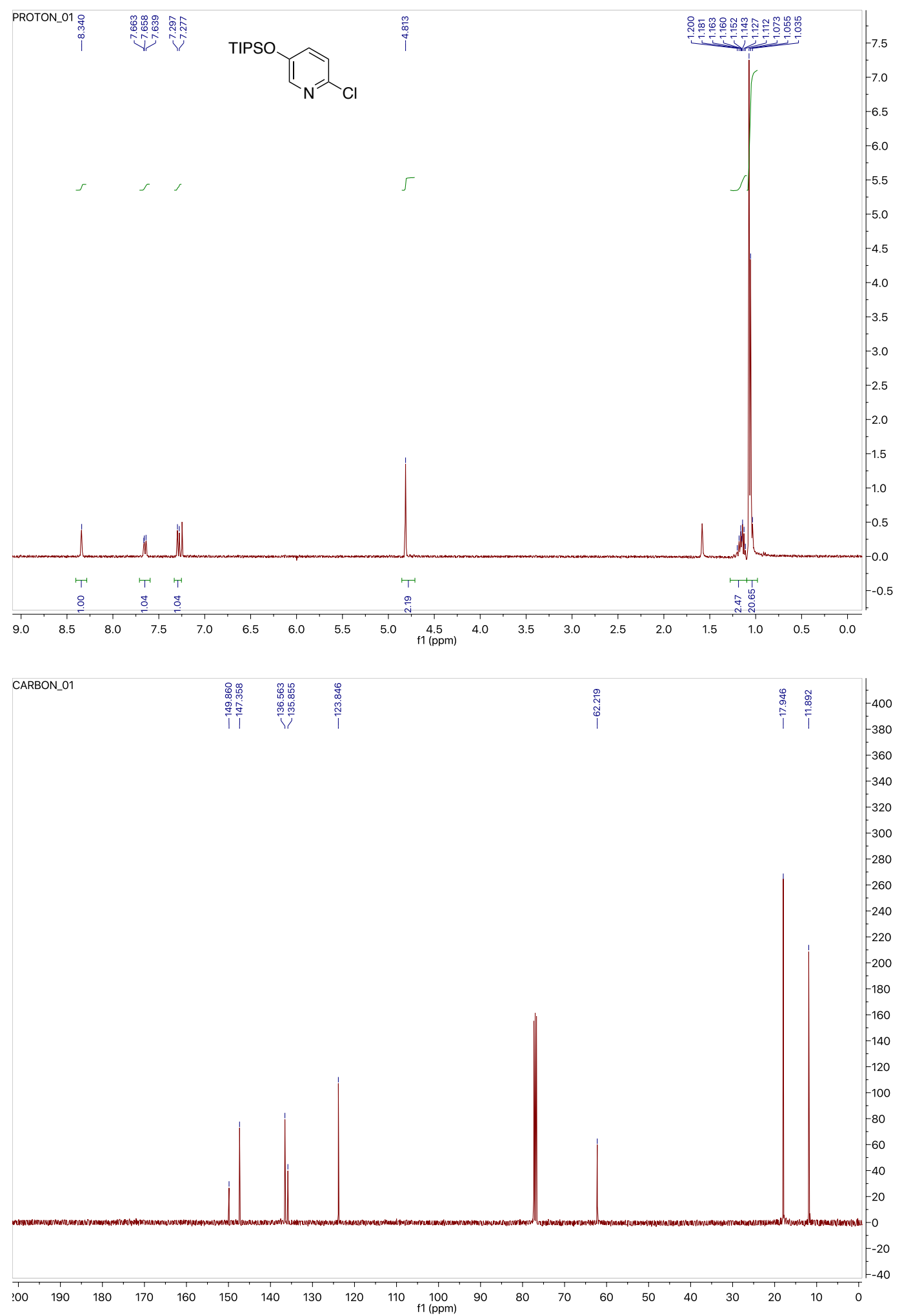
S33 Supporting Information. Rossler, Hartgerink, Zerull, Anderson et. al.

\section{Compound 10d}
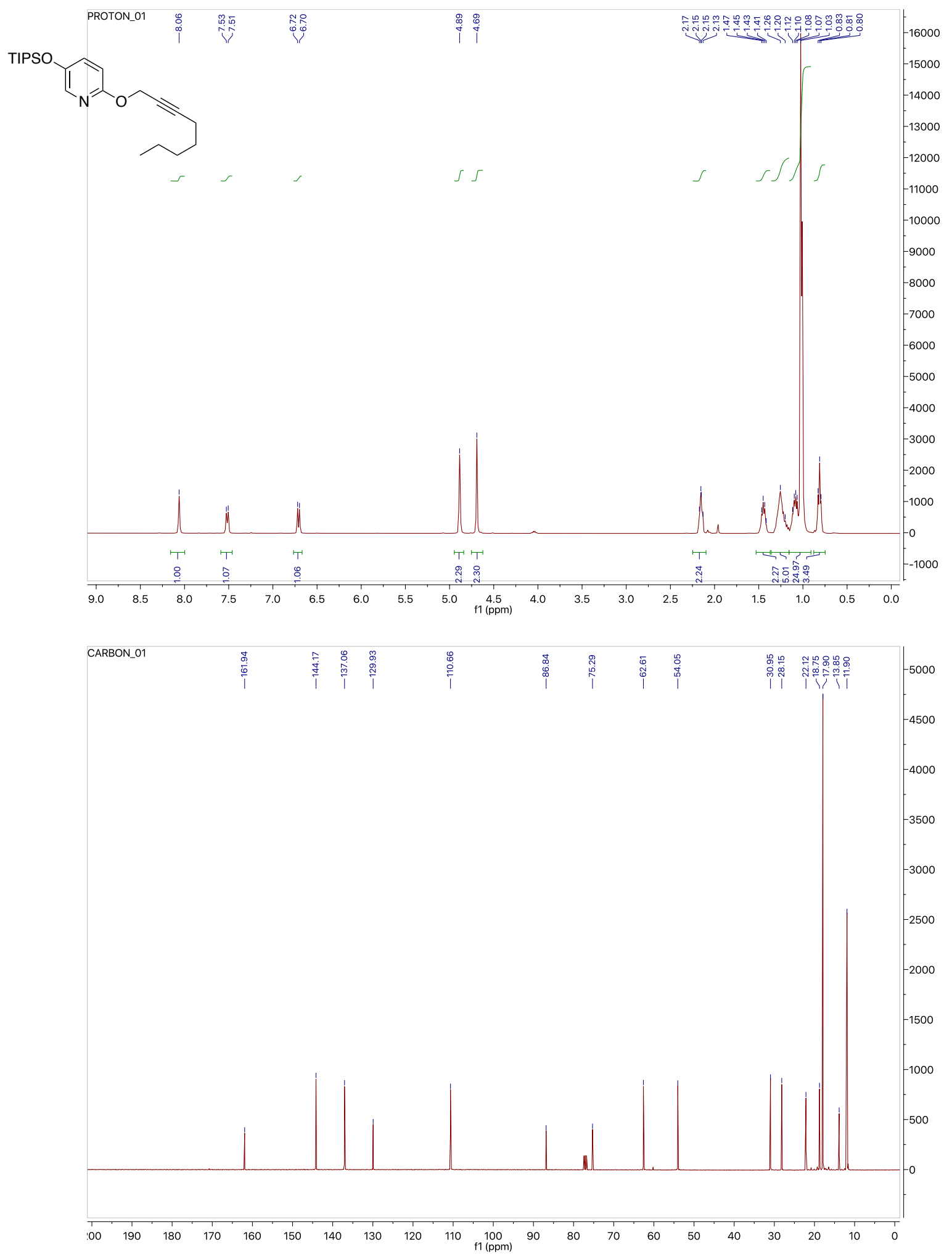
S34 Supporting Information. Rossler, Hartgerink, Zerull, Anderson et. al.

\section{Compound 4}

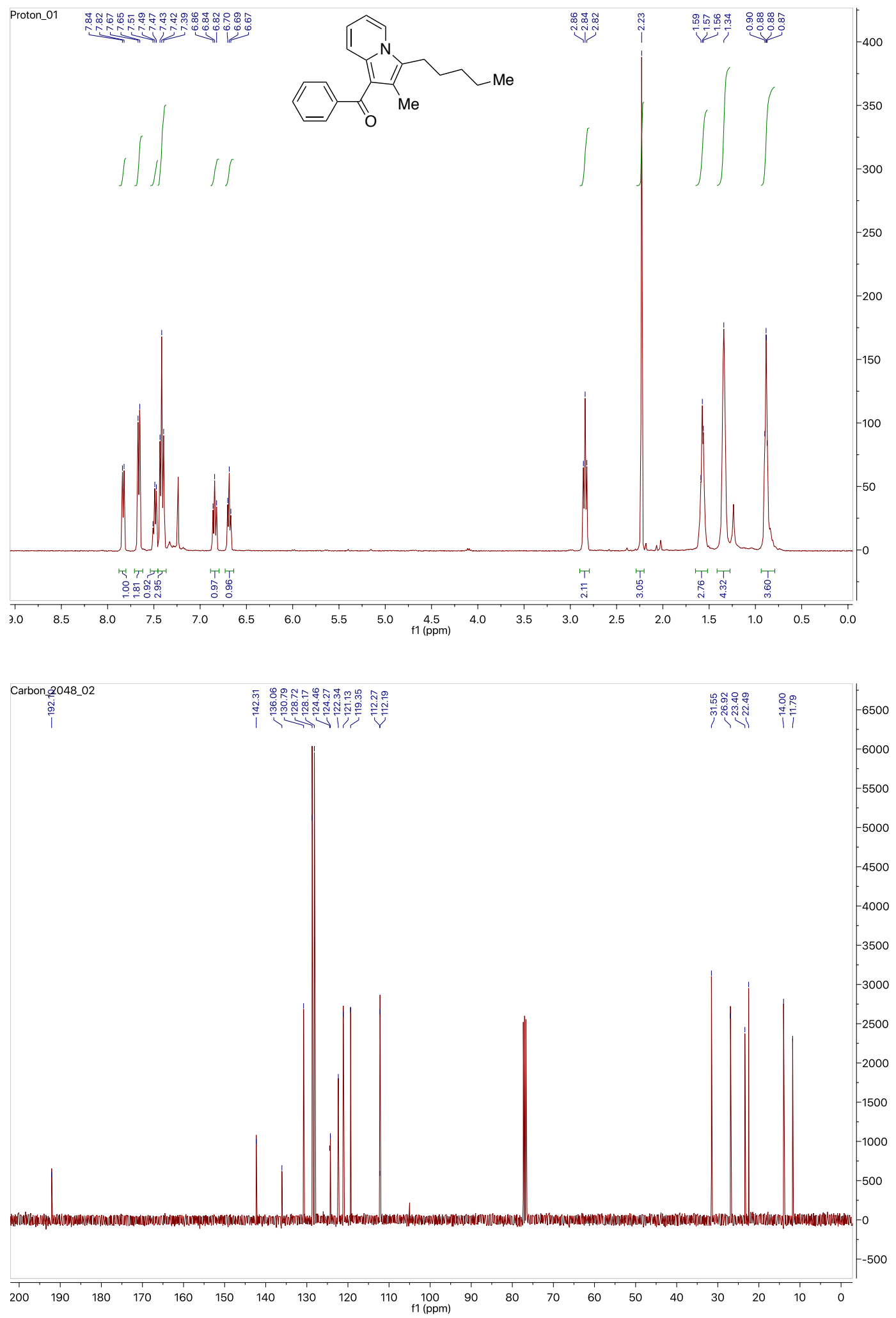


S35 Supporting Information. Rossler, Hartgerink, Zerull, Anderson et. al.

\section{Compound 7a}

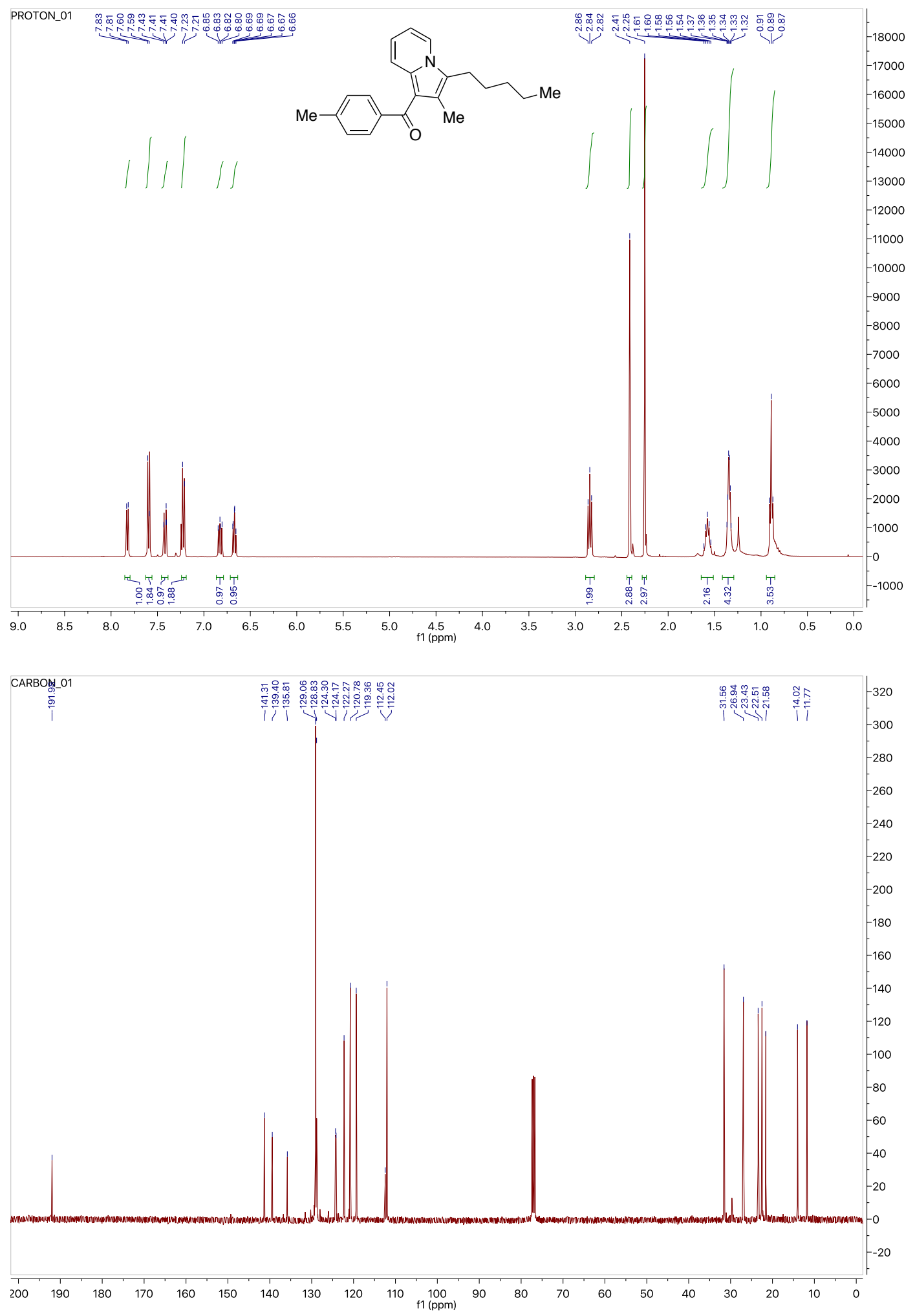


S36 Supporting Information. Rossler, Hartgerink, Zerull, Anderson et. al.

\section{Compound $7 \mathrm{~b}$}
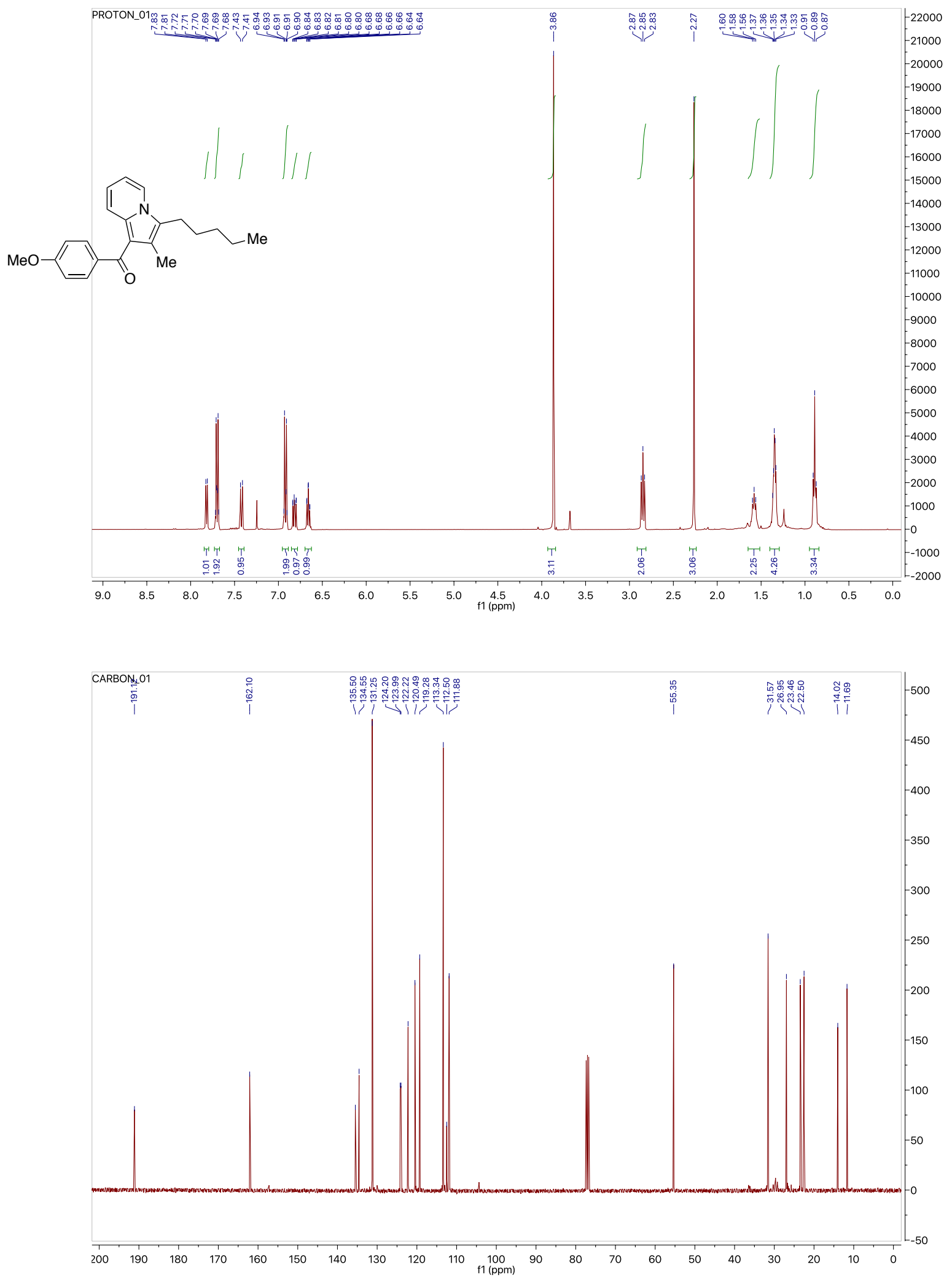
S37 Supporting Information. Rossler, Hartgerink, Zerull, Anderson et. al.

\section{Compound 7c}
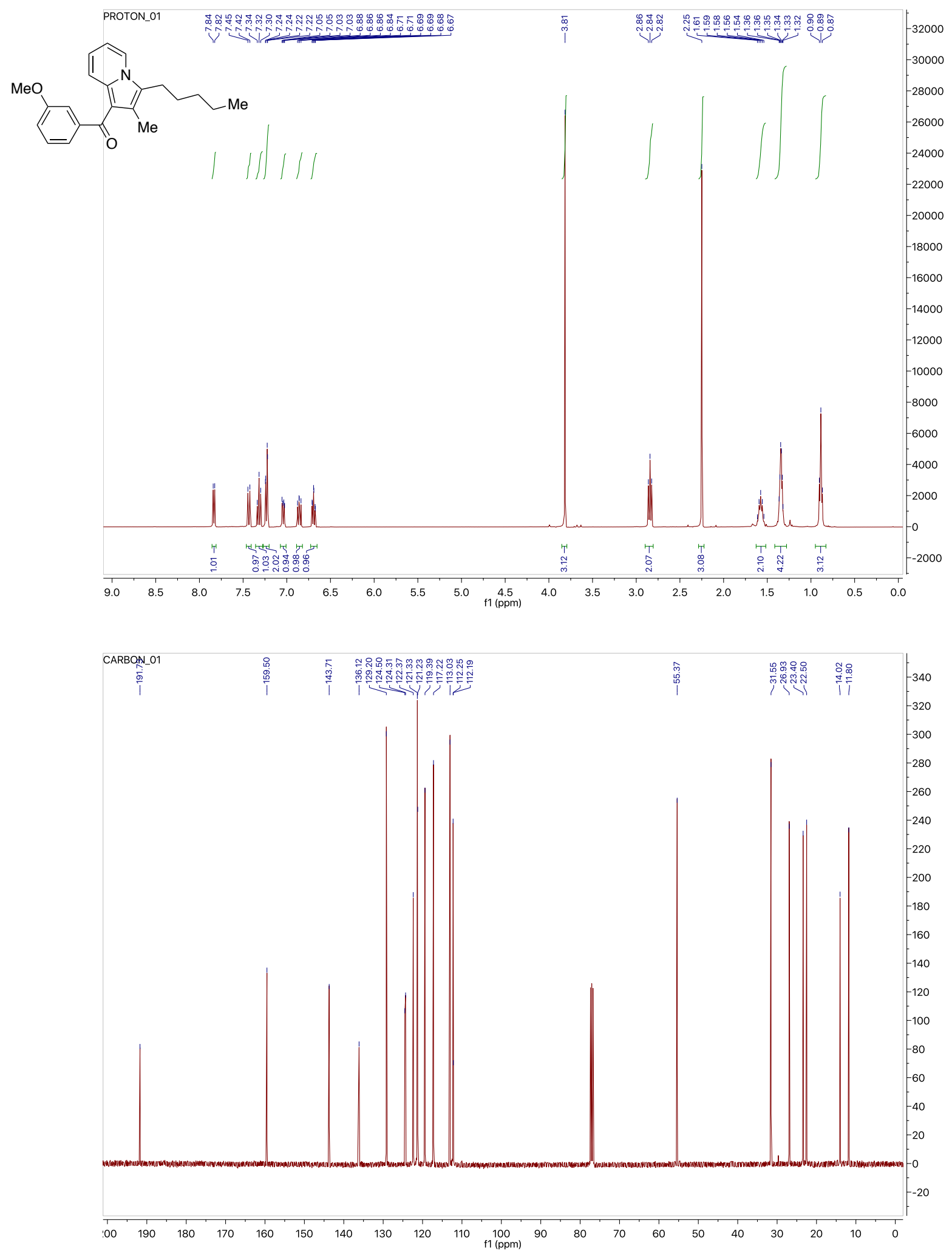
S38 Supporting Information. Rossler, Hartgerink, Zerull, Anderson et. al.

\section{Compound 7d}

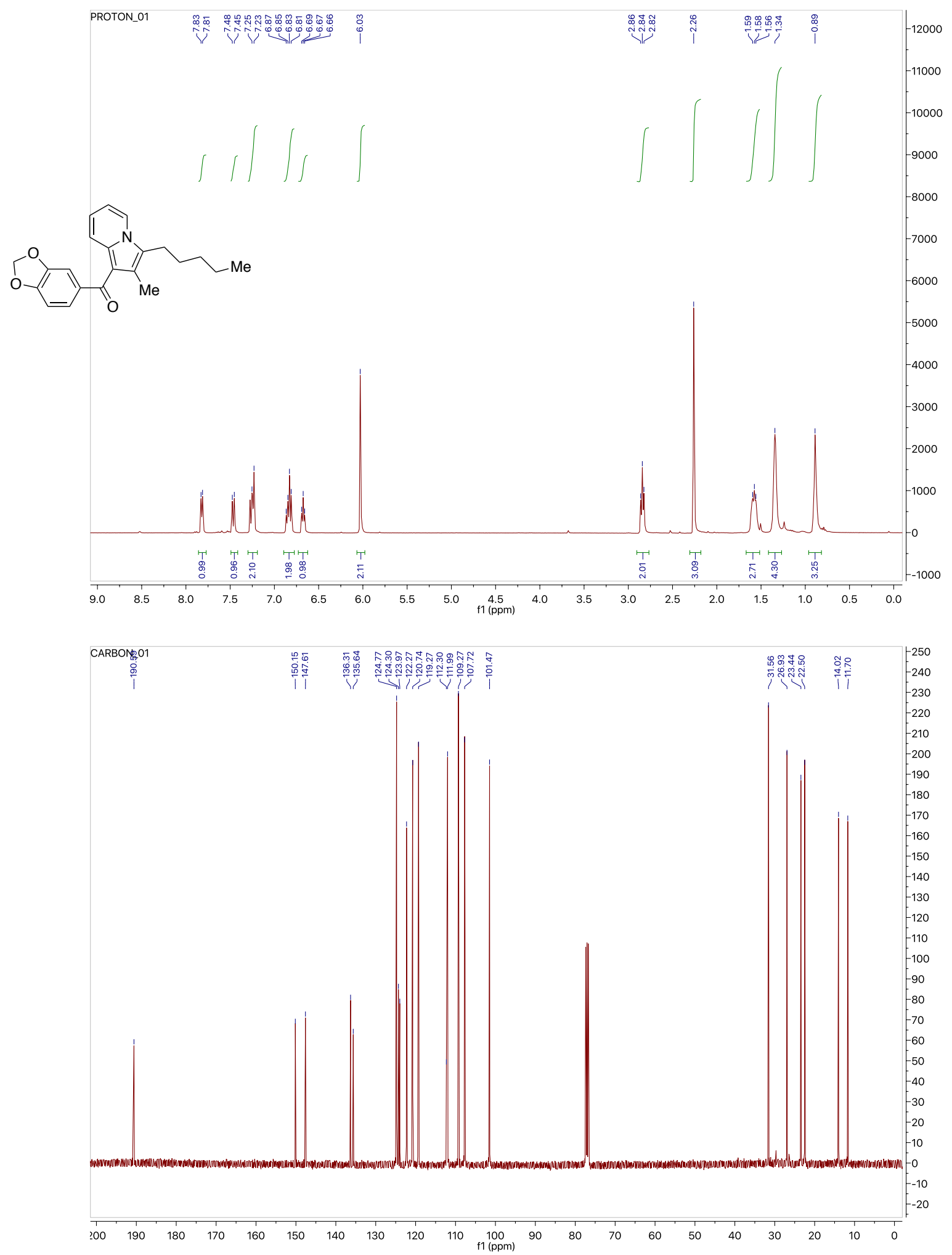


S39 Supporting Information. Rossler, Hartgerink, Zerull, Anderson et. al.

\section{Compound 7e}
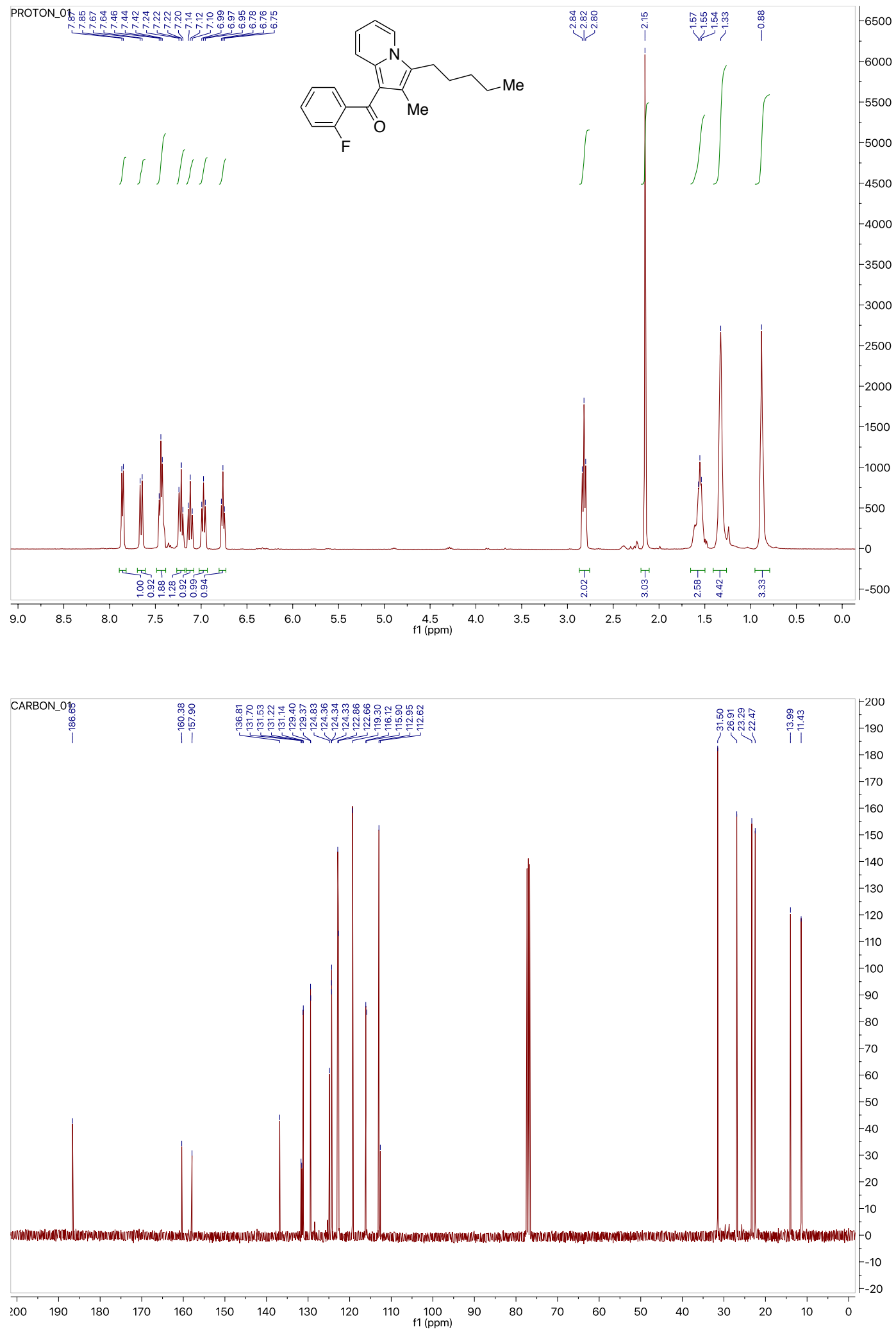
S40 Supporting Information. Rossler, Hartgerink, Zerull, Anderson et. al.

\section{Compound 7f}
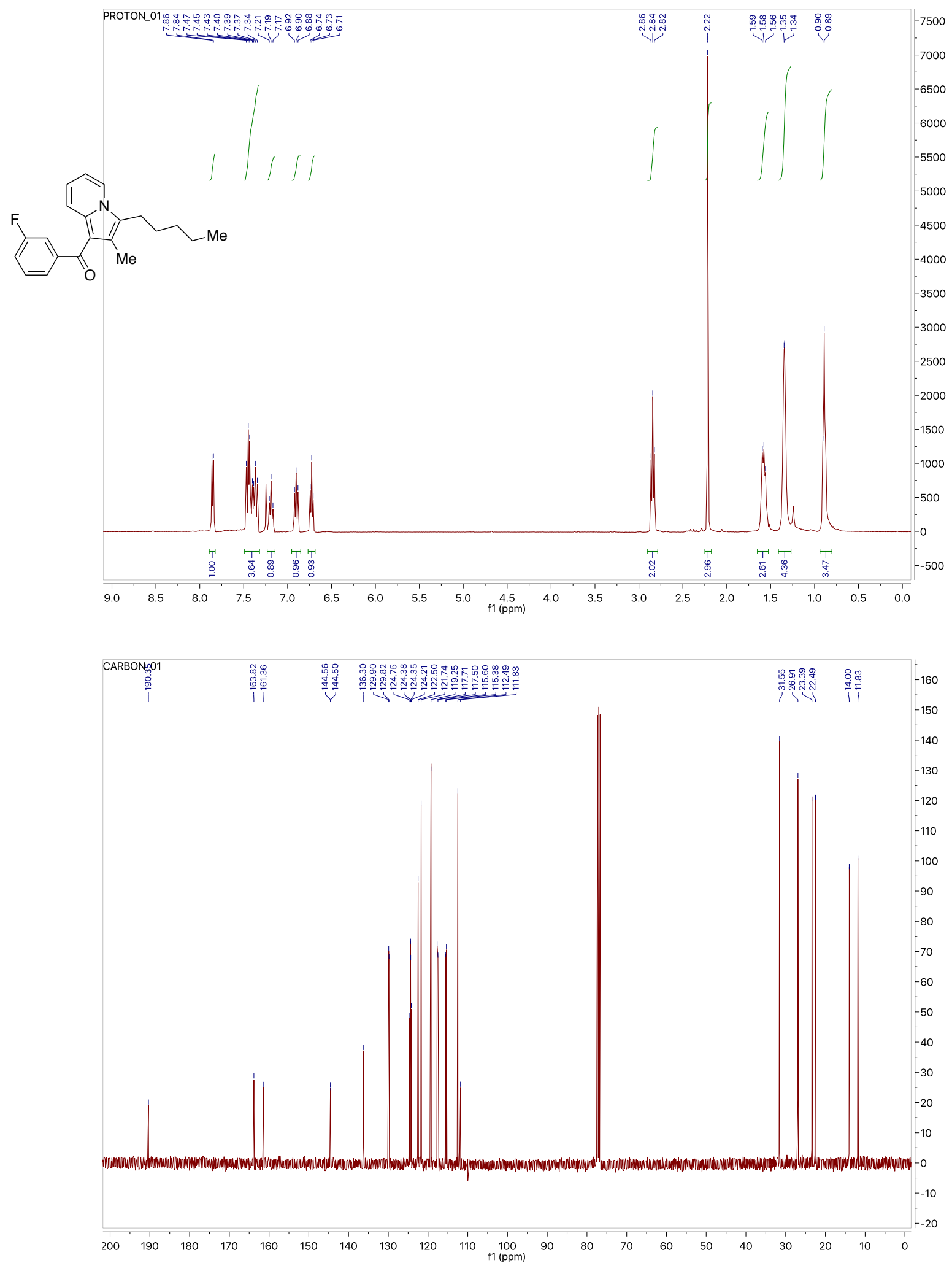
S41 Supporting Information. Rossler, Hartgerink, Zerull, Anderson et. al.

\section{Compound $7 \mathrm{~g}$}
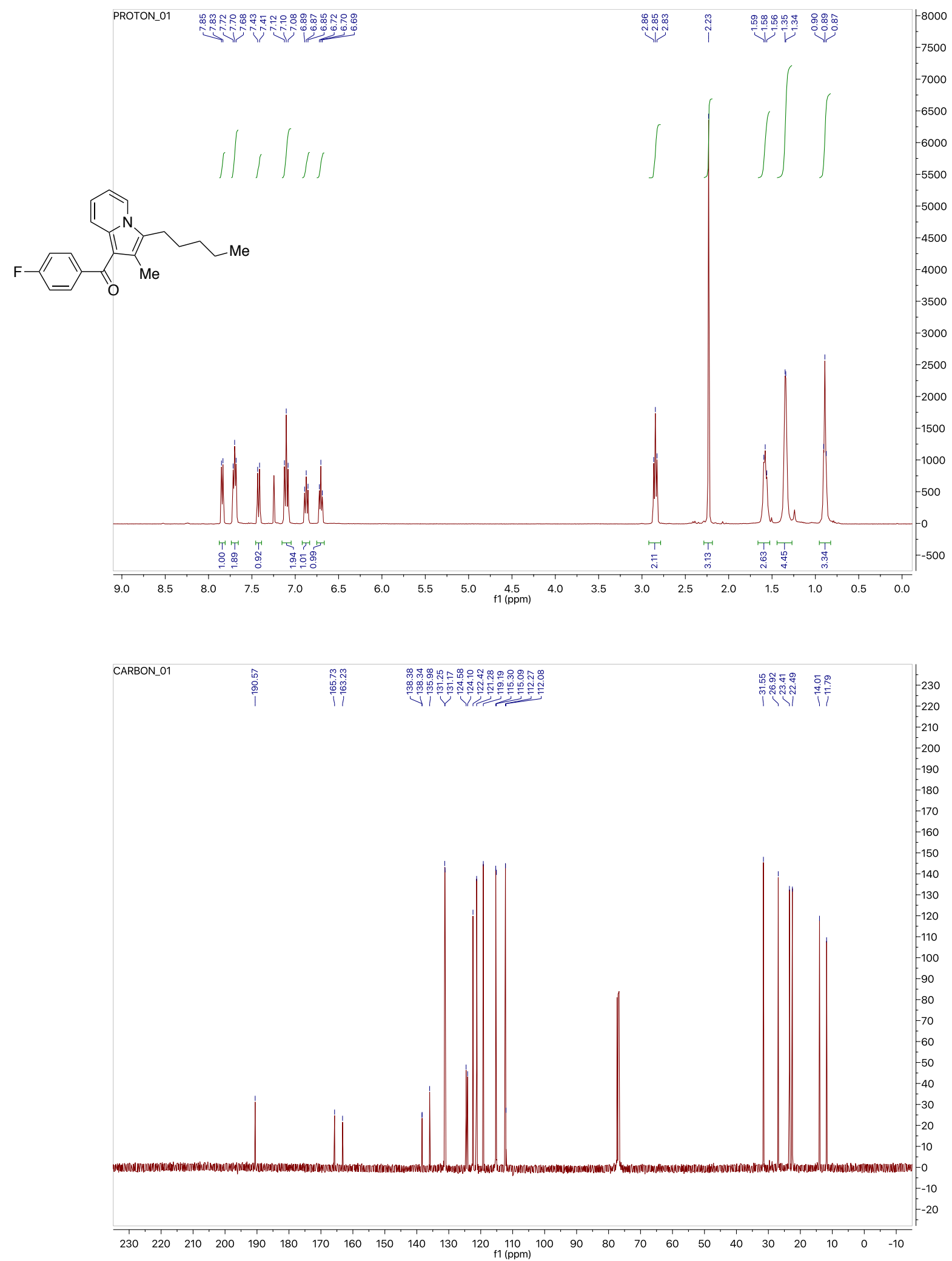
S42 Supporting Information. Rossler, Hartgerink, Zerull, Anderson et. al.

\section{Compound 7h}

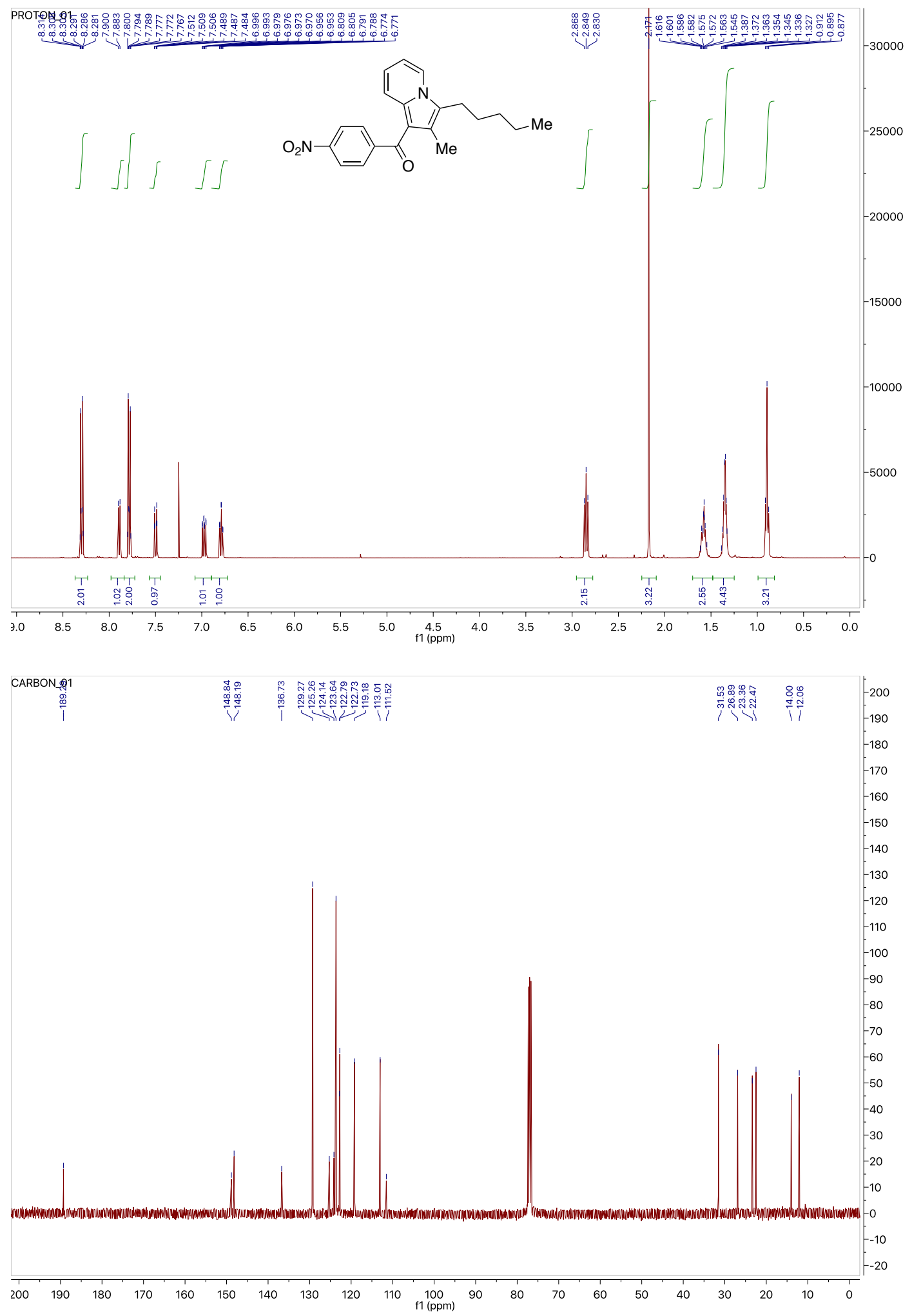


S43 Supporting Information. Rossler, Hartgerink, Zerull, Anderson et. al.

\section{Compound 7i}
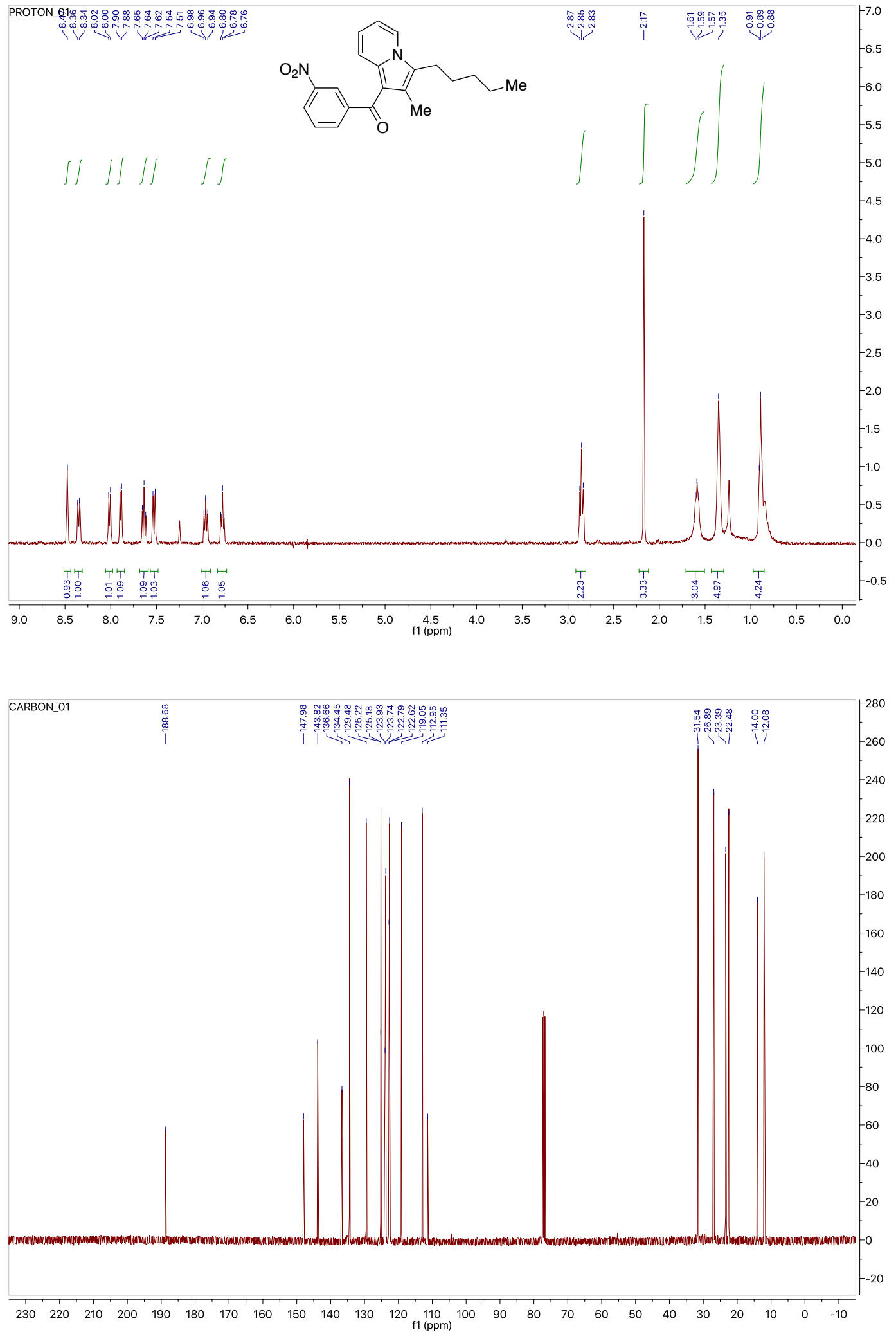
S44 Supporting Information. Rossler, Hartgerink, Zerull, Anderson et. al.

\section{Compound $7 \mathrm{j}$}
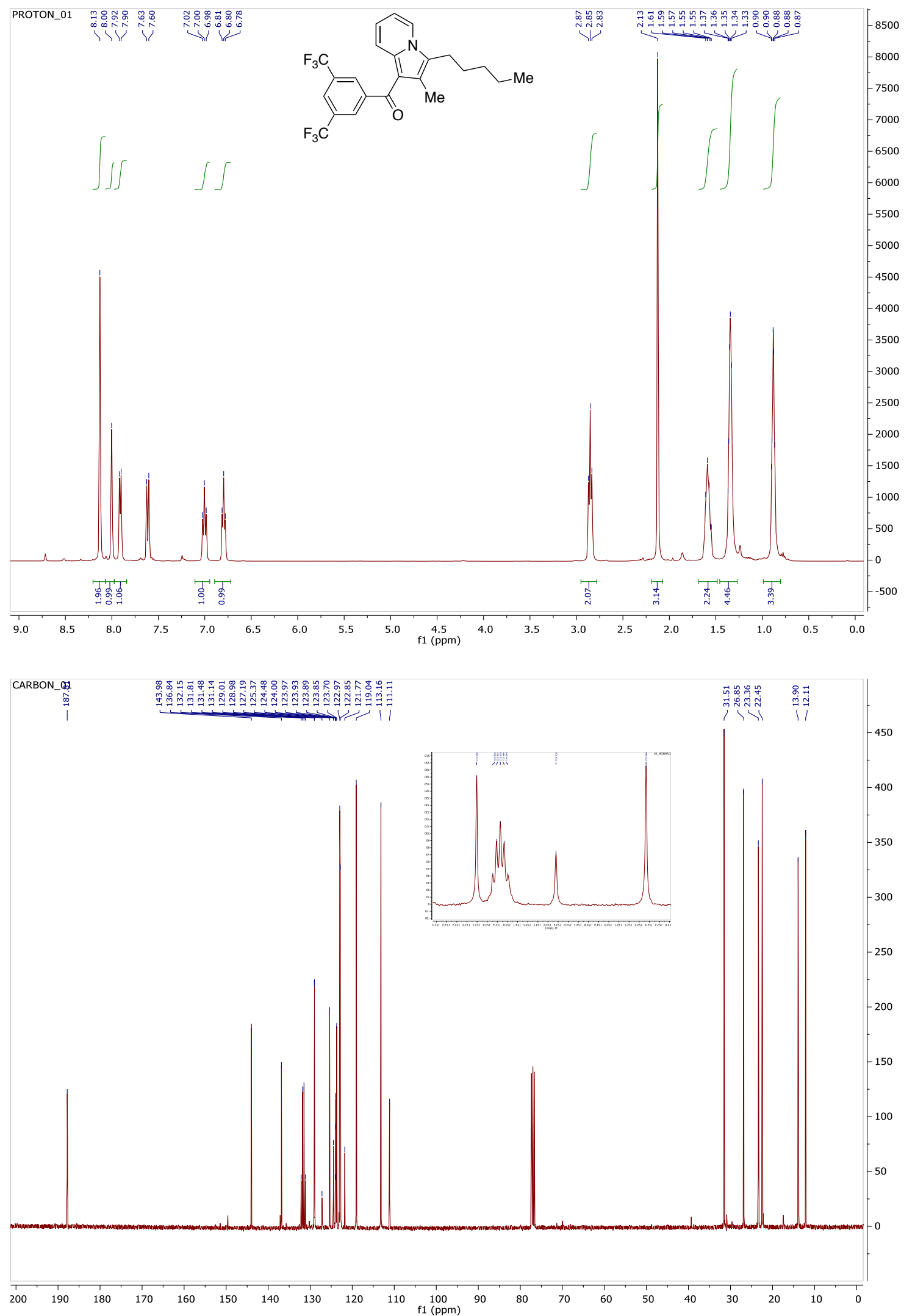
S45 Supporting Information. Rossler, Hartgerink, Zerull, Anderson et. al.

\section{Compound 7k}
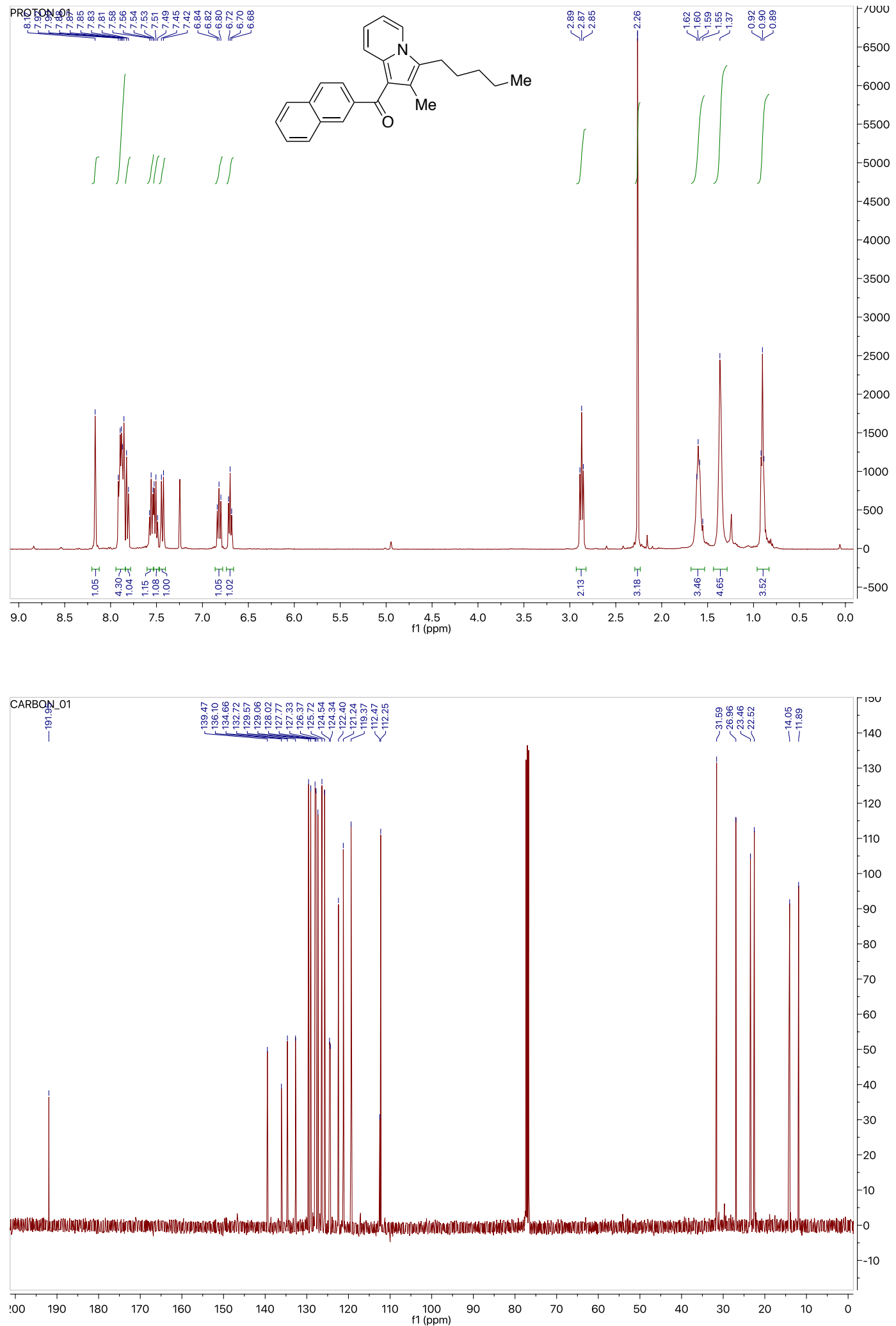
S46 Supporting Information. Rossler, Hartgerink, Zerull, Anderson et. al.

\section{Compound 71}
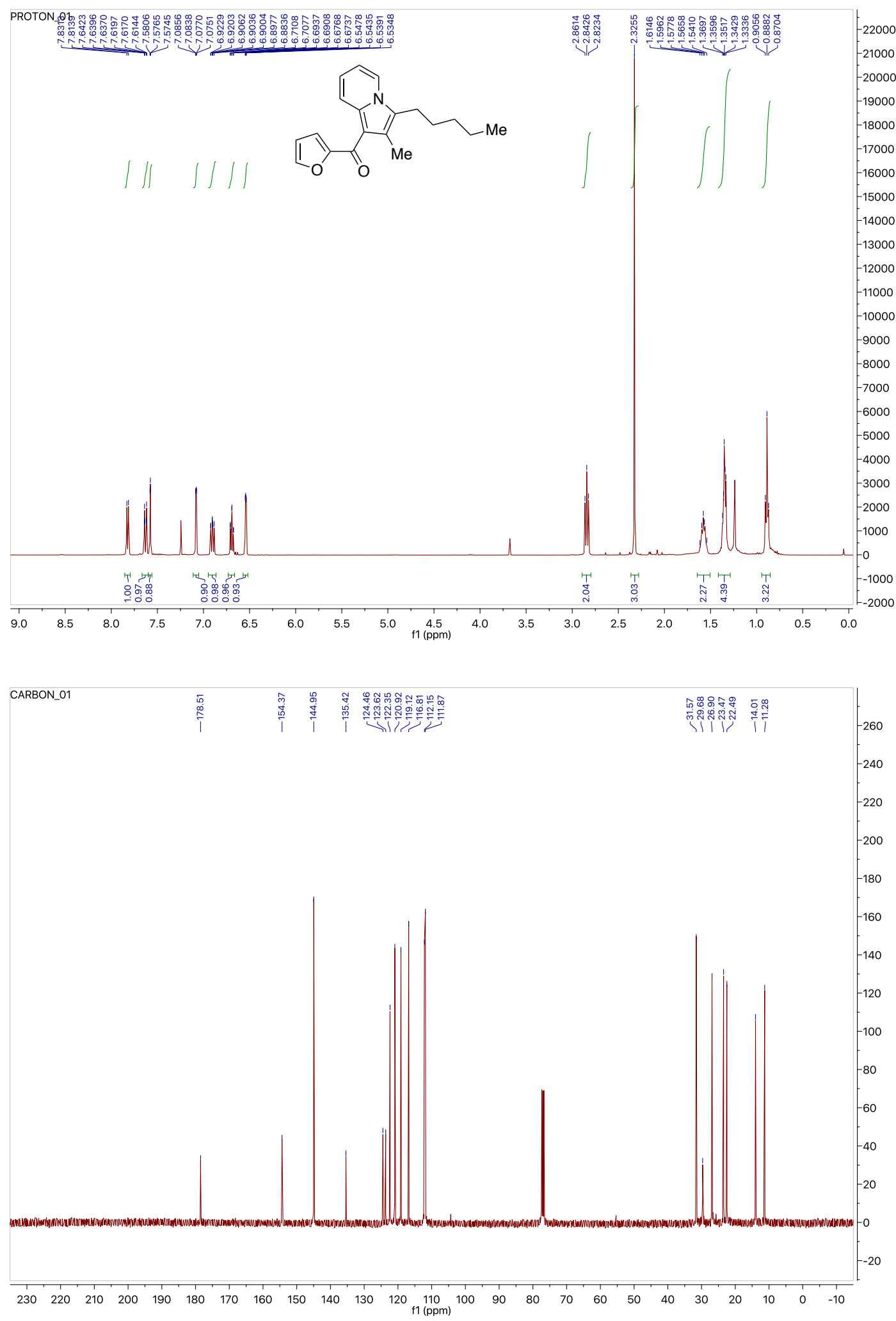
S47 Supporting Information. Rossler, Hartgerink, Zerull, Anderson et. al.

\section{Compound $7 \mathrm{~m}$}

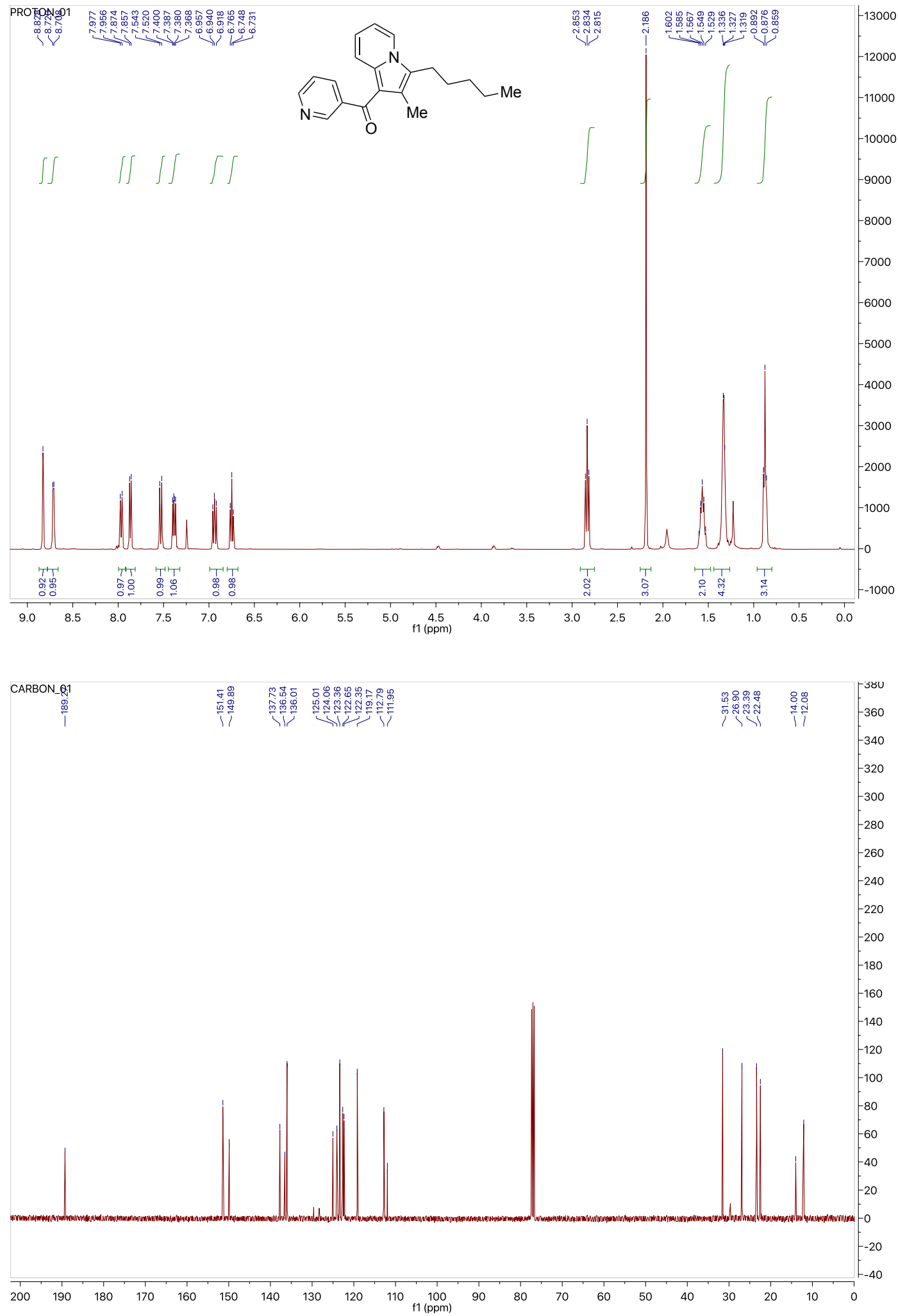


S48 Supporting Information. Rossler, Hartgerink, Zerull, Anderson et. al.

\section{Compound 7n}

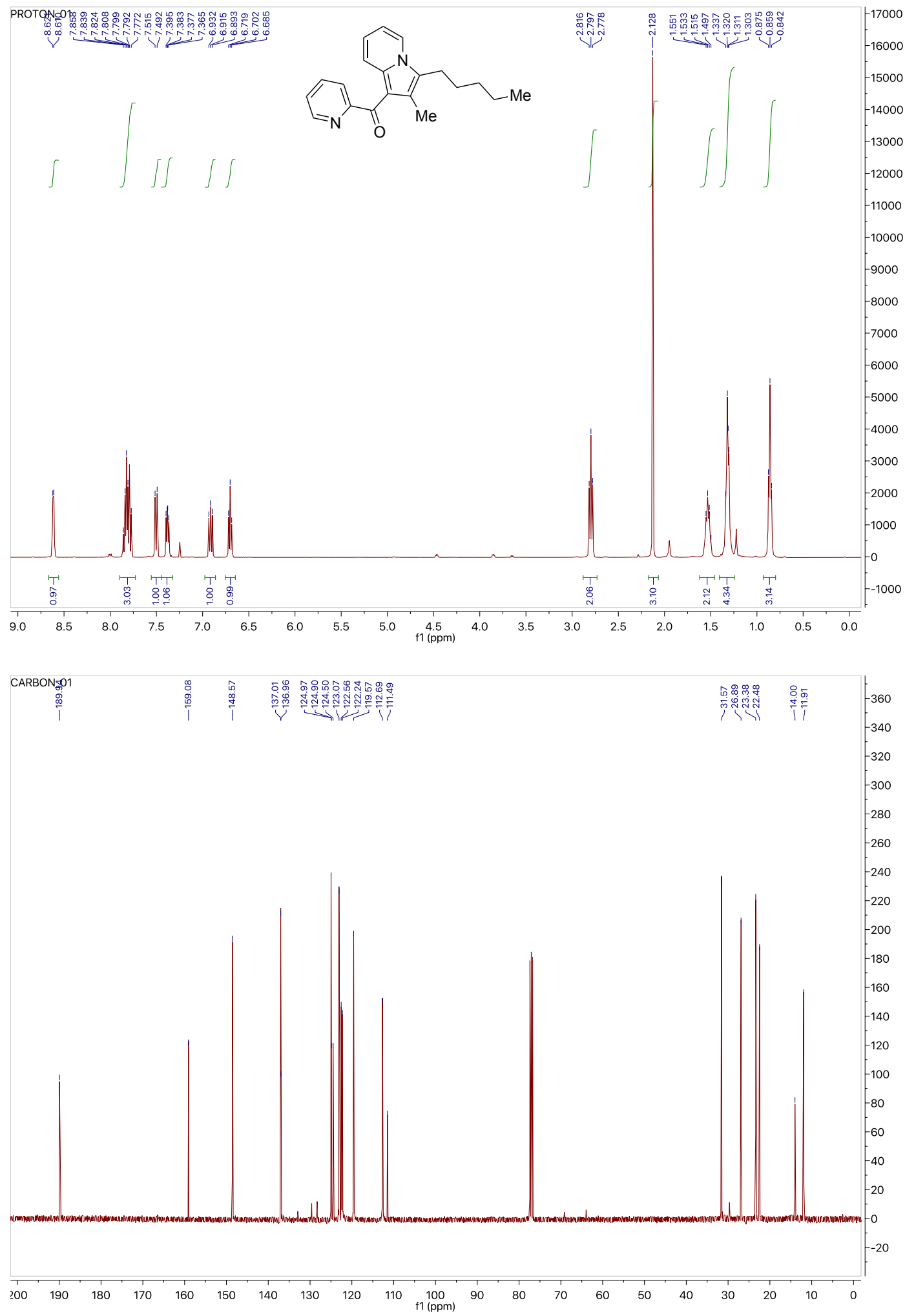


S49 Supporting Information. Rossler, Hartgerink, Zerull, Anderson et. al.

\section{Compound 9a}
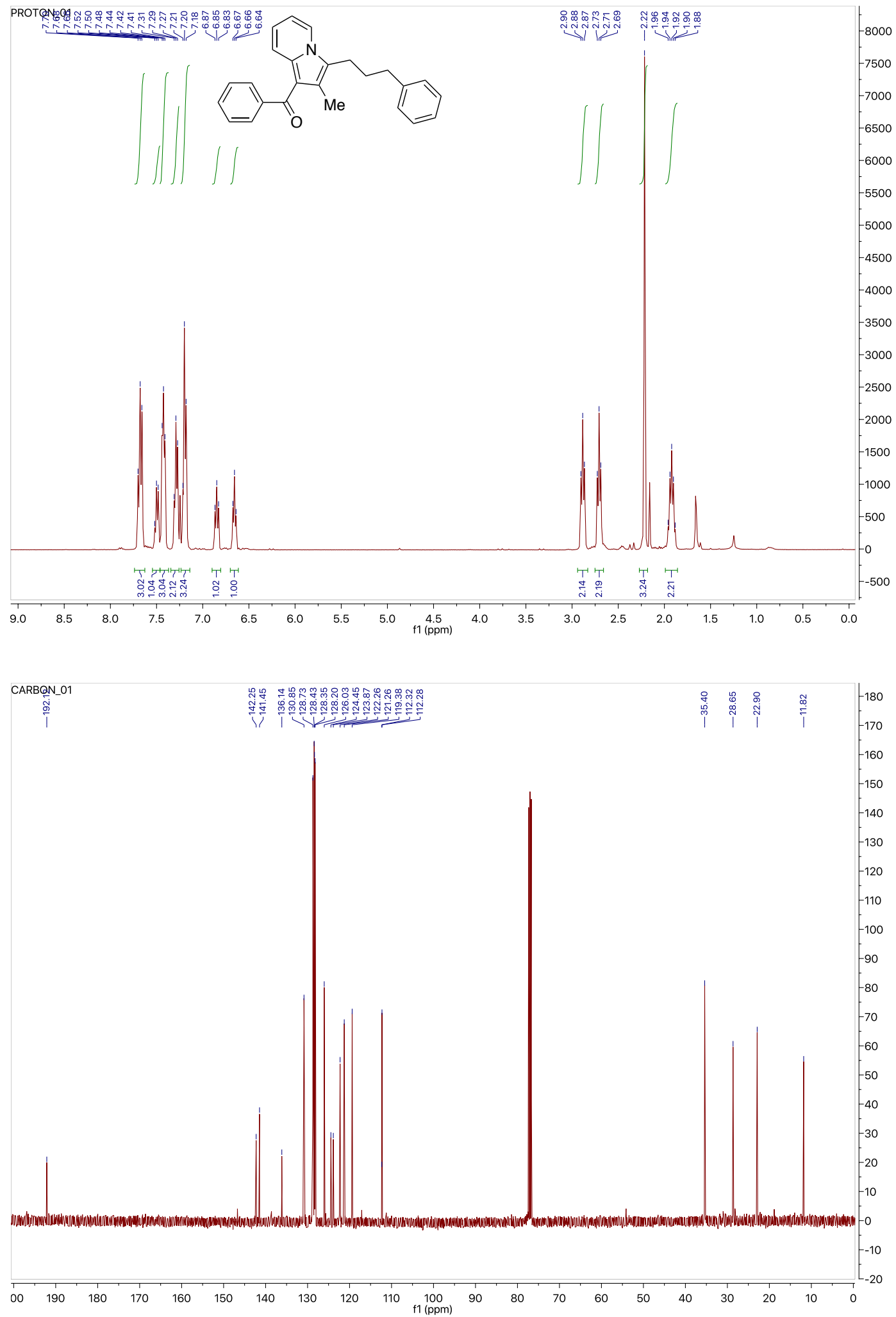
S50 Supporting Information. Rossler, Hartgerink, Zerull, Anderson et. al.

\section{Compound 9aa}
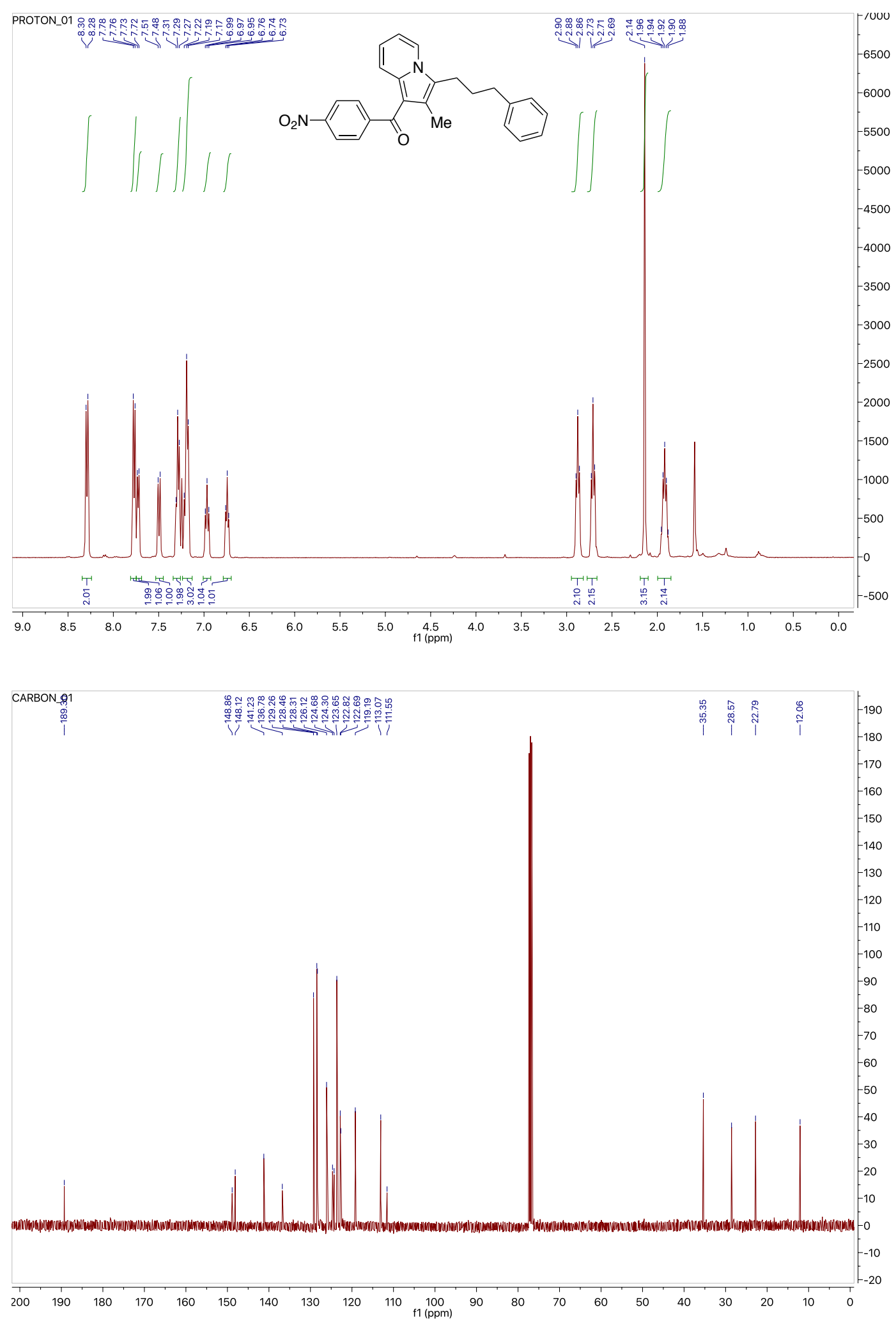
S51 Supporting Information. Rossler, Hartgerink, Zerull, Anderson et. al.

\section{Compound 9b}

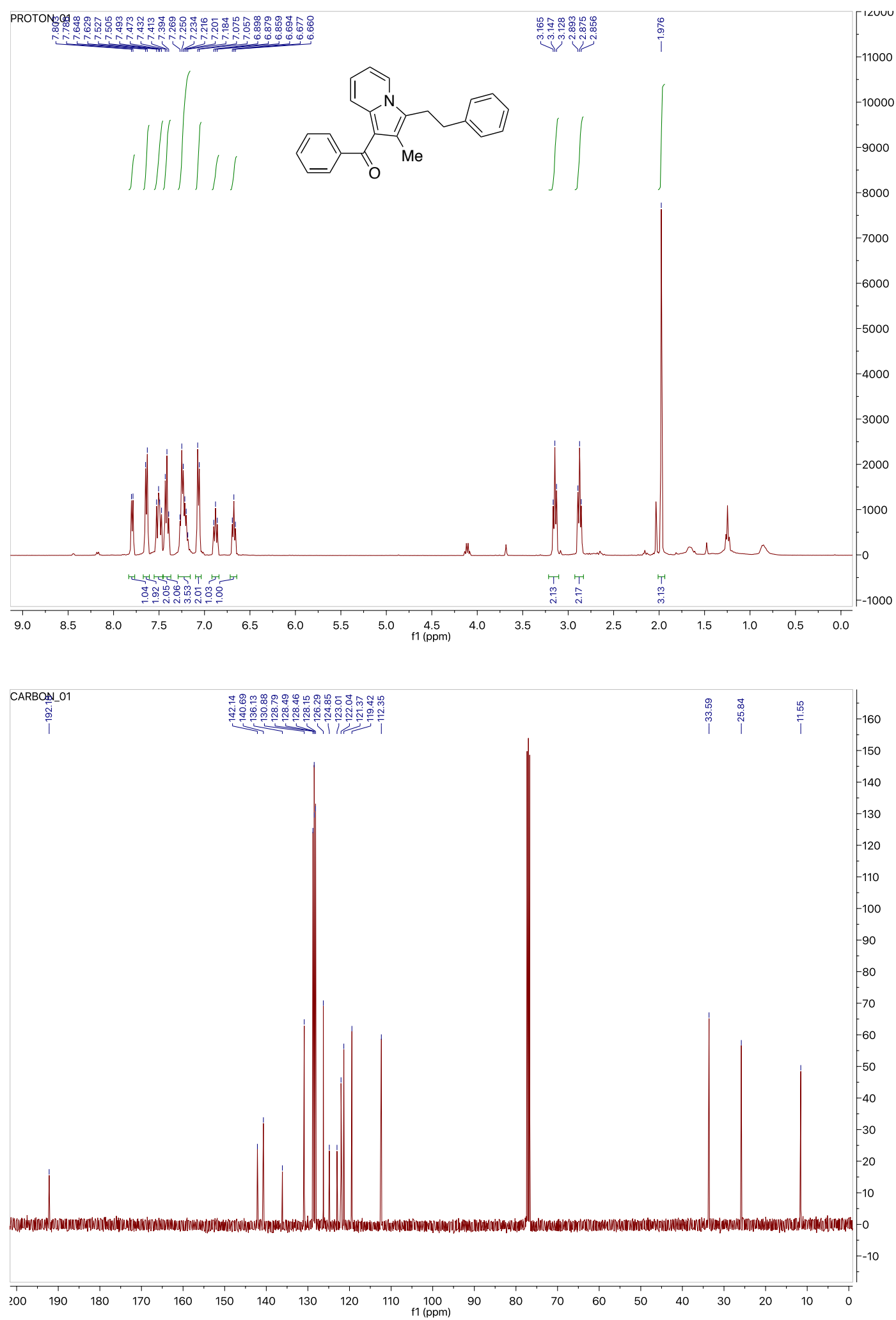


S52 Supporting Information. Rossler, Hartgerink, Zerull, Anderson et. al.

\section{Compound 9bb}

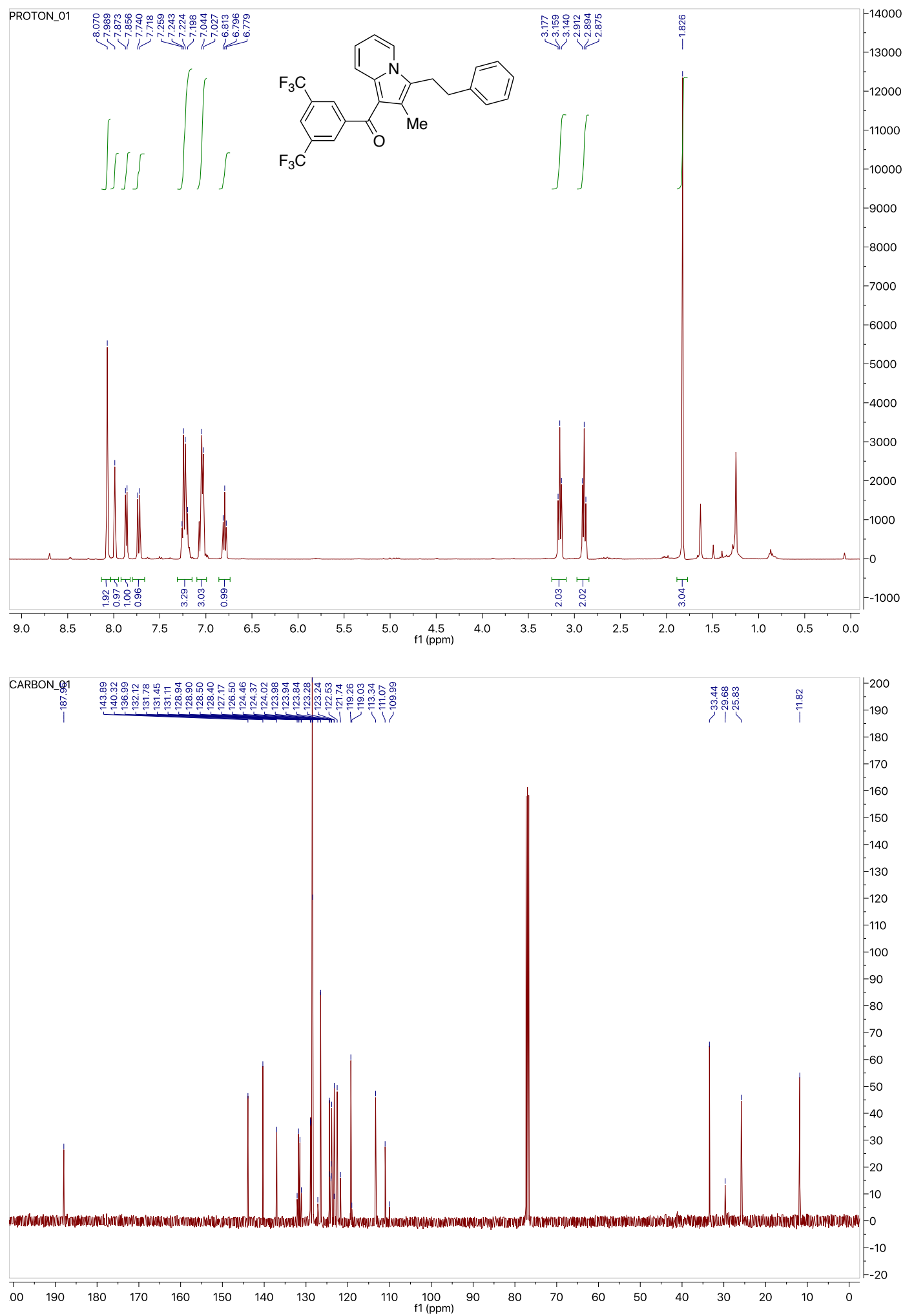


S53 Supporting Information. Rossler, Hartgerink, Zerull, Anderson et. al.

\section{Compound 9c}
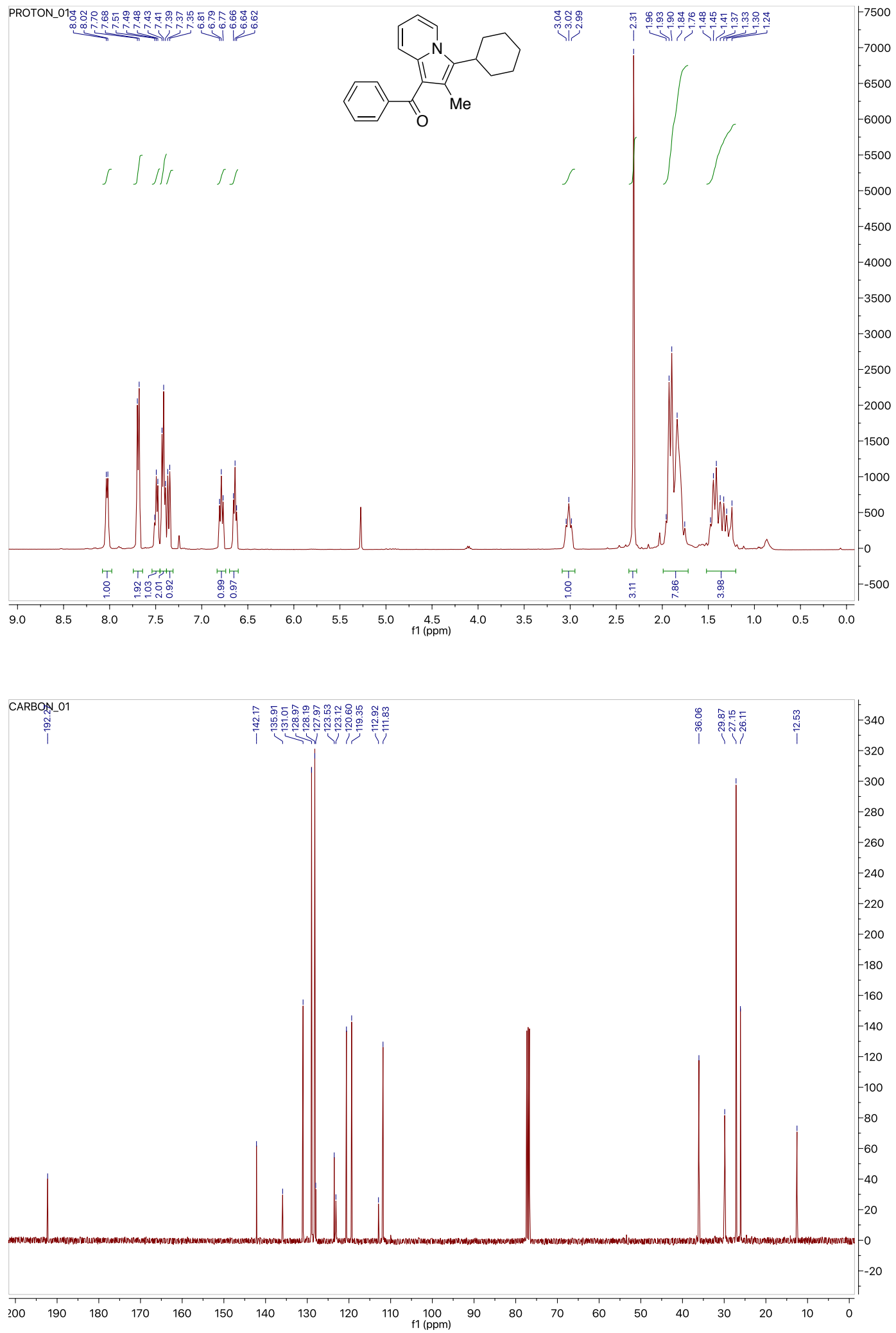
S54 Supporting Information. Rossler, Hartgerink, Zerull, Anderson et. al.

\section{Compound 9ce}

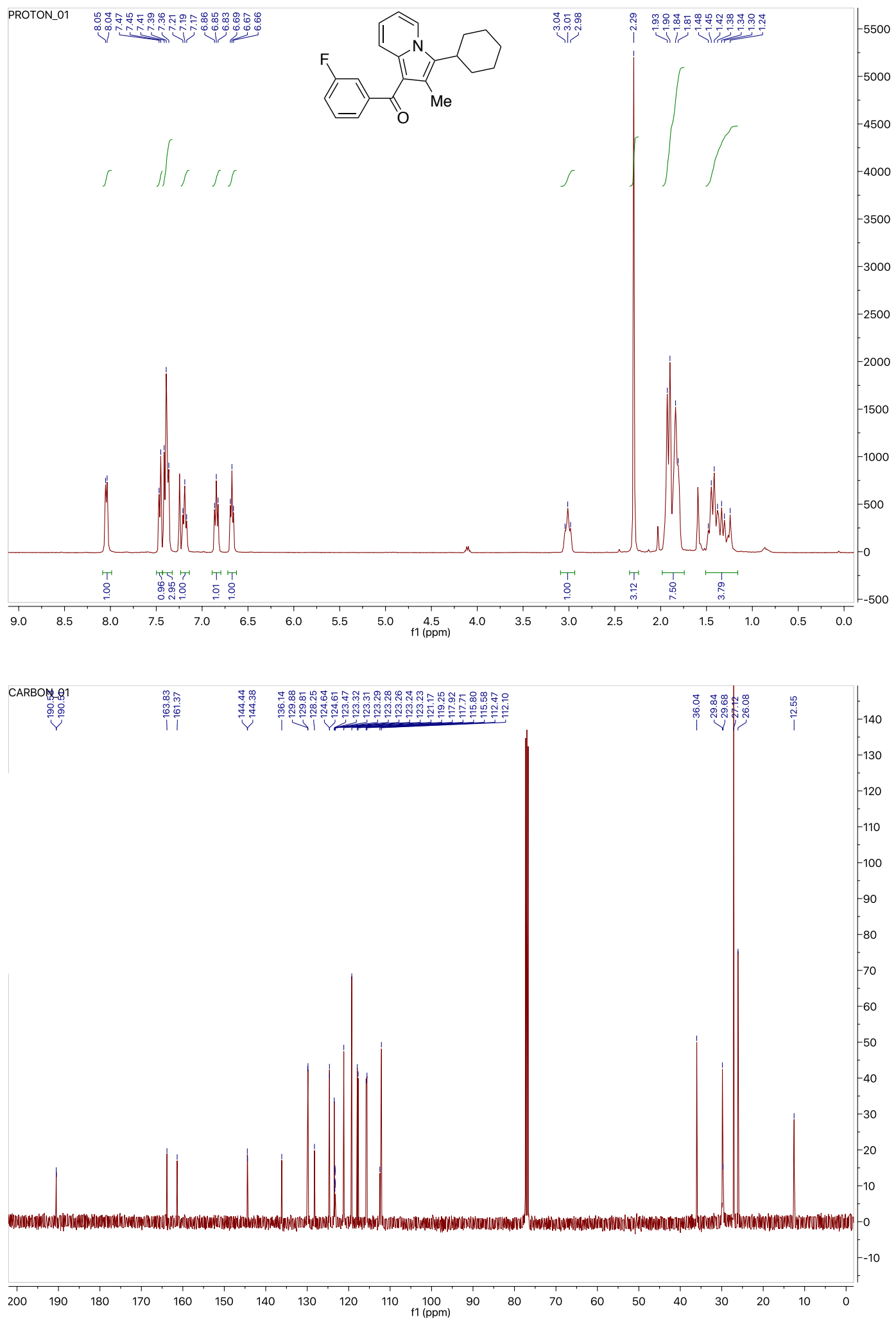


S55 Supporting Information. Rossler, Hartgerink, Zerull, Anderson et. al.

\section{Compound 9cec}
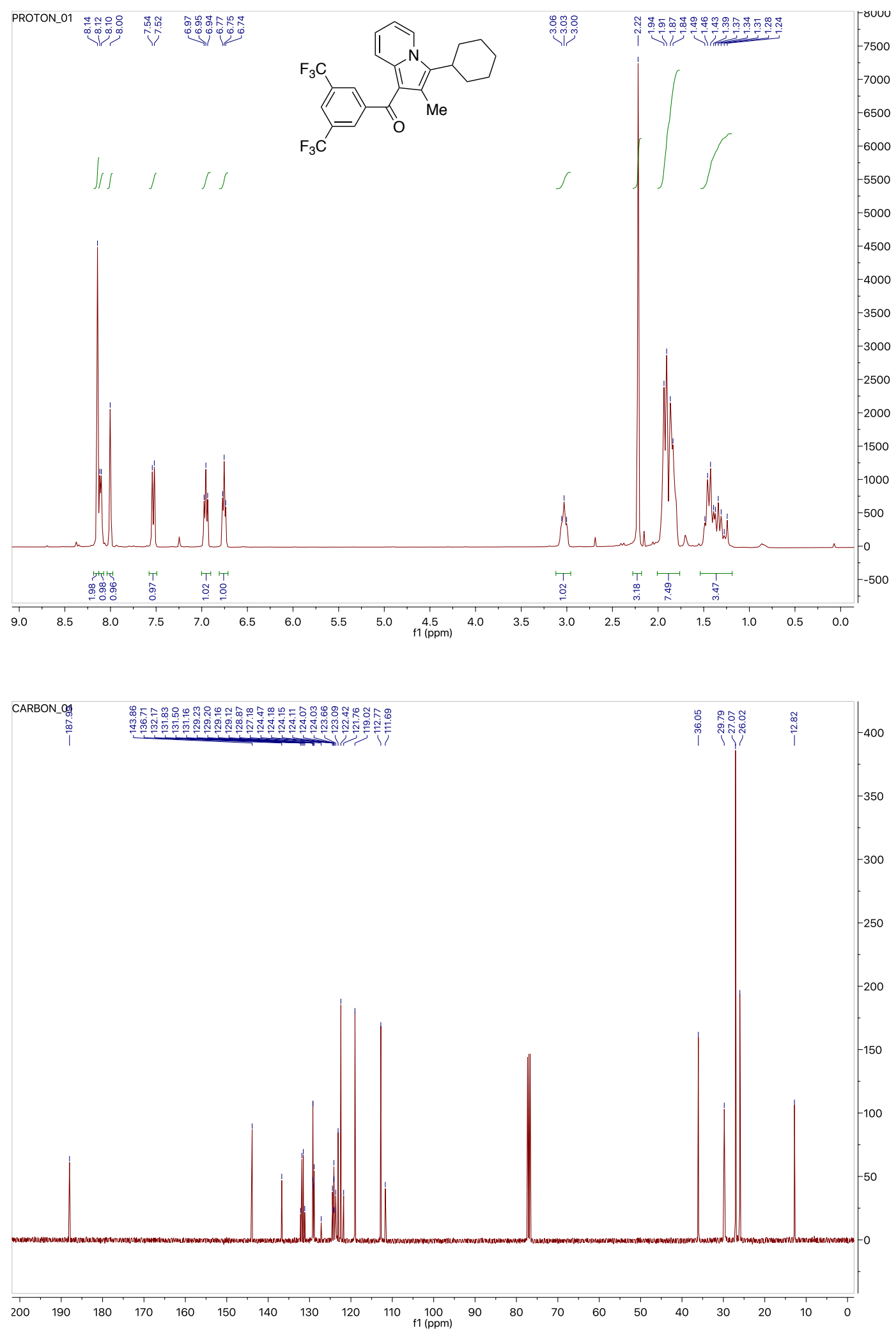
S56 Supporting Information. Rossler, Hartgerink, Zerull, Anderson et. al.

\section{Compound 9d}
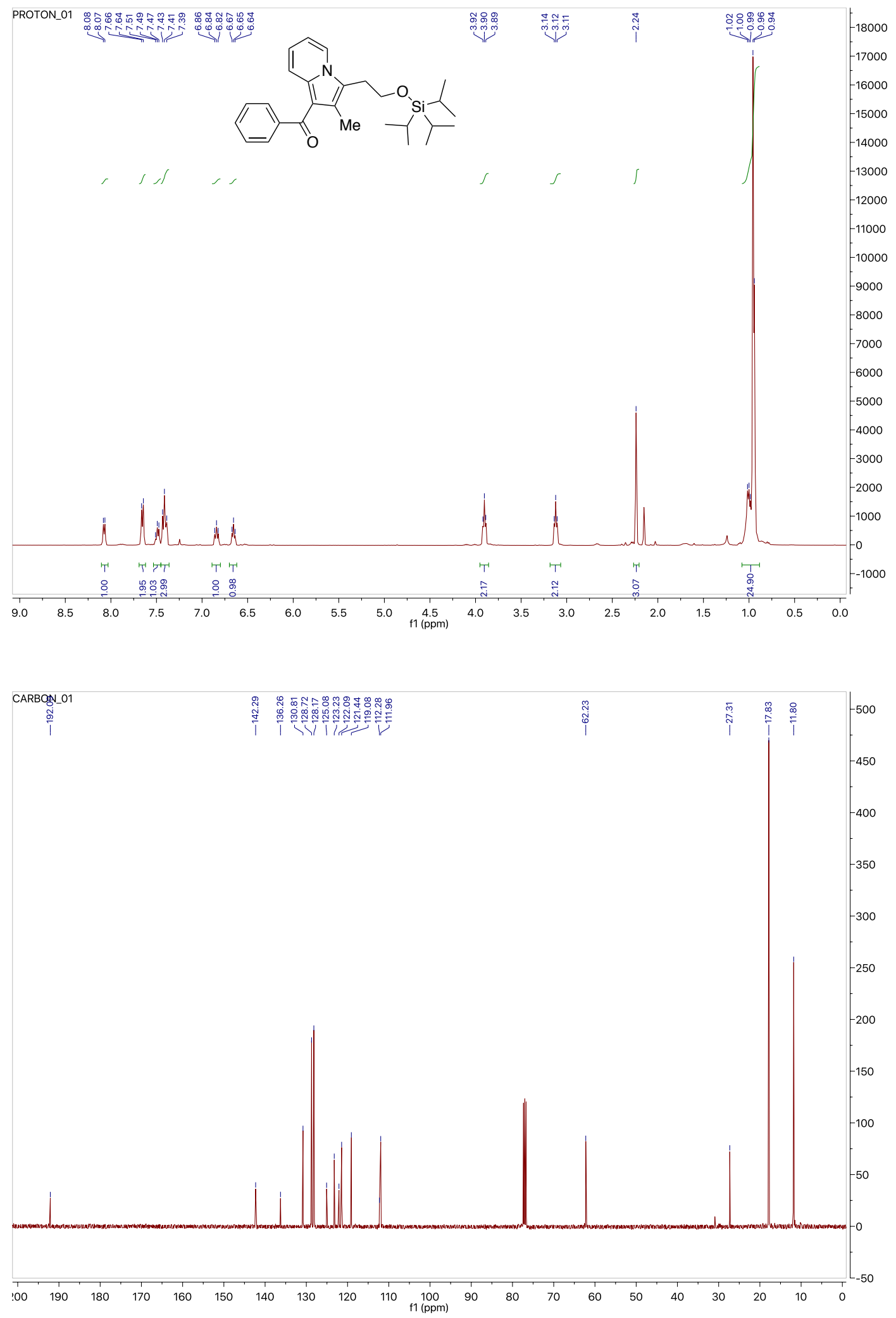
S57 Supporting Information. Rossler, Hartgerink, Zerull, Anderson et. al.

\section{Compound 9dd}
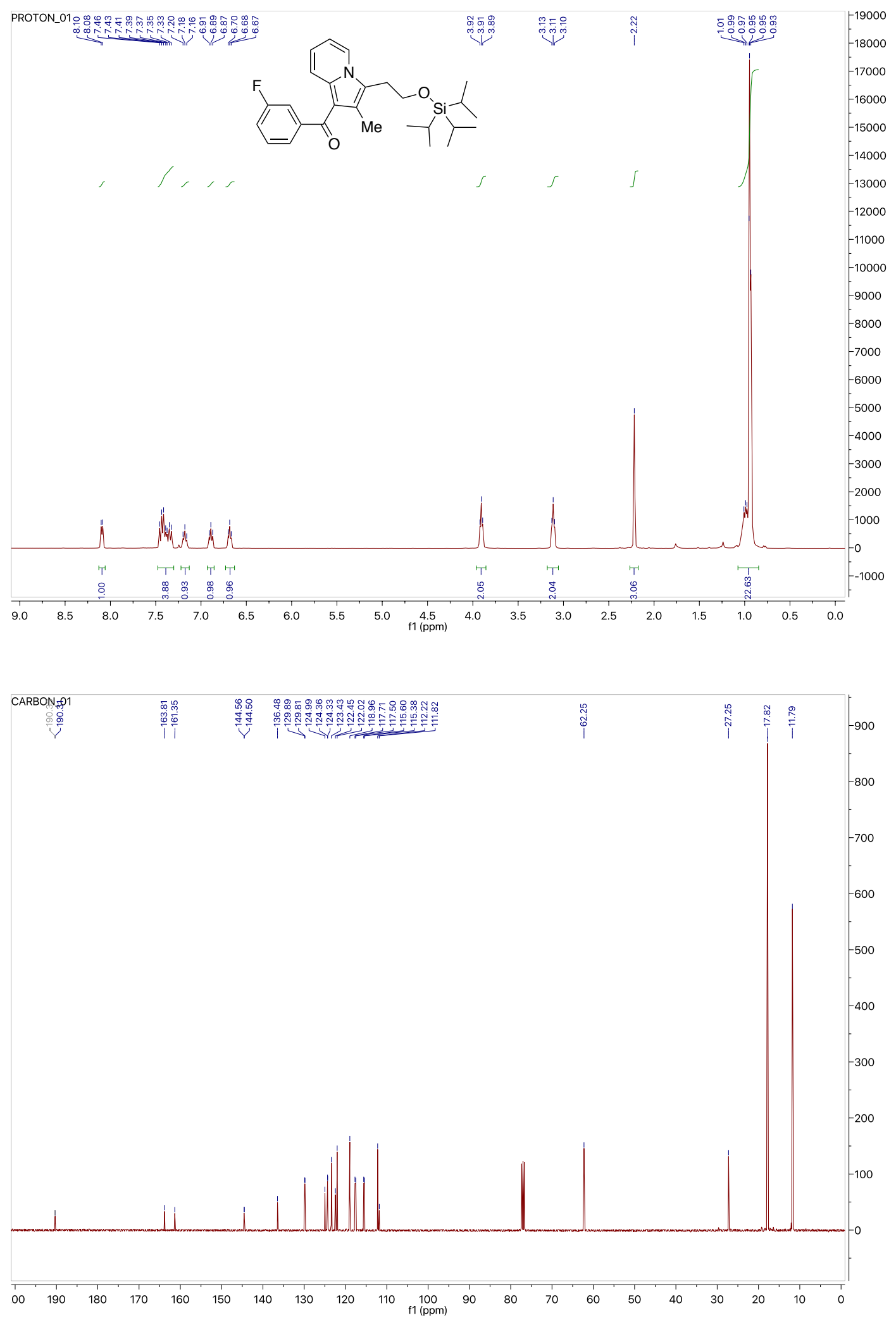
S58 Supporting Information. Rossler, Hartgerink, Zerull, Anderson et. al.

\section{Compound 9e}

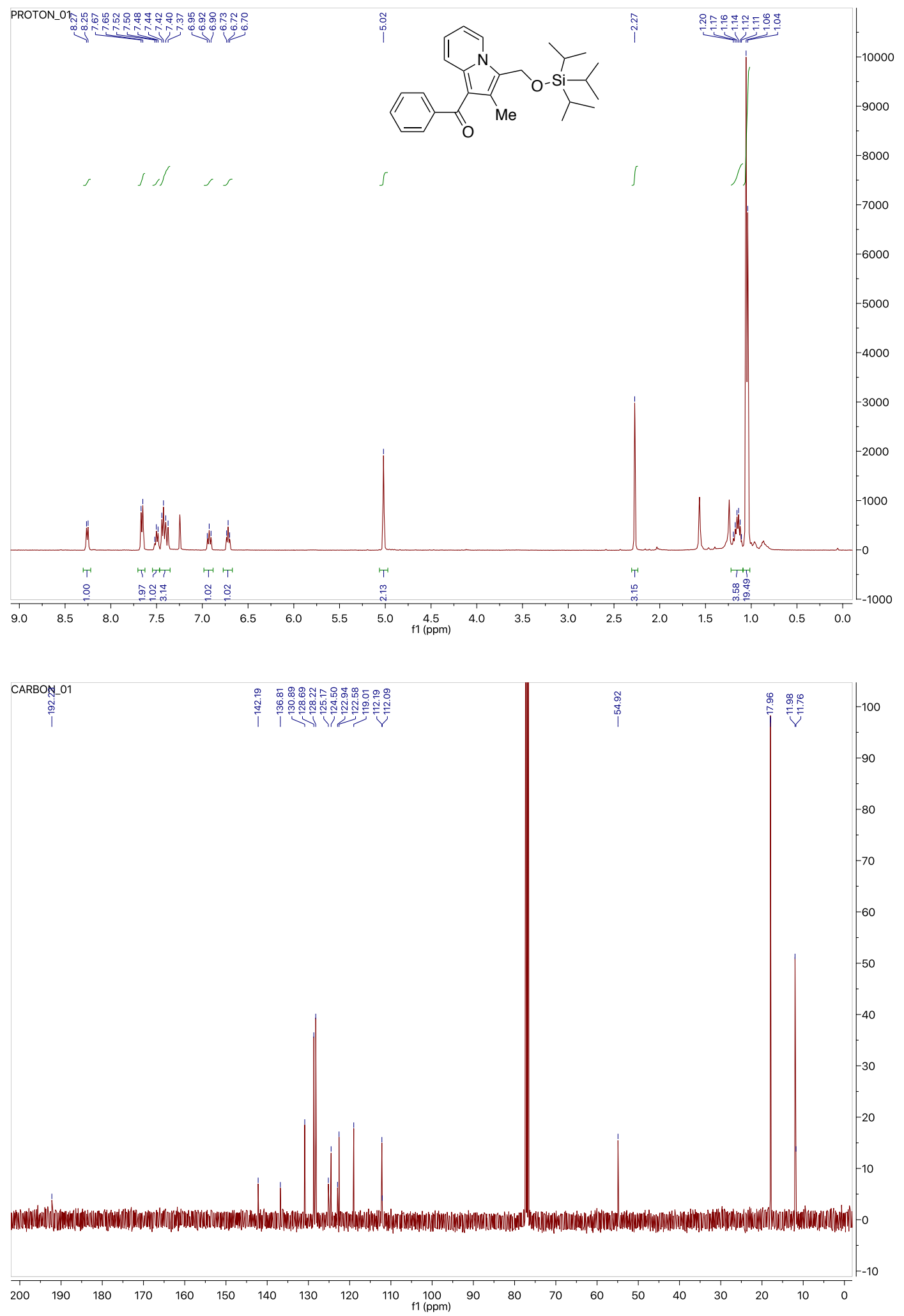


S59 Supporting Information. Rossler, Hartgerink, Zerull, Anderson et. al.

\section{Compound 9f}
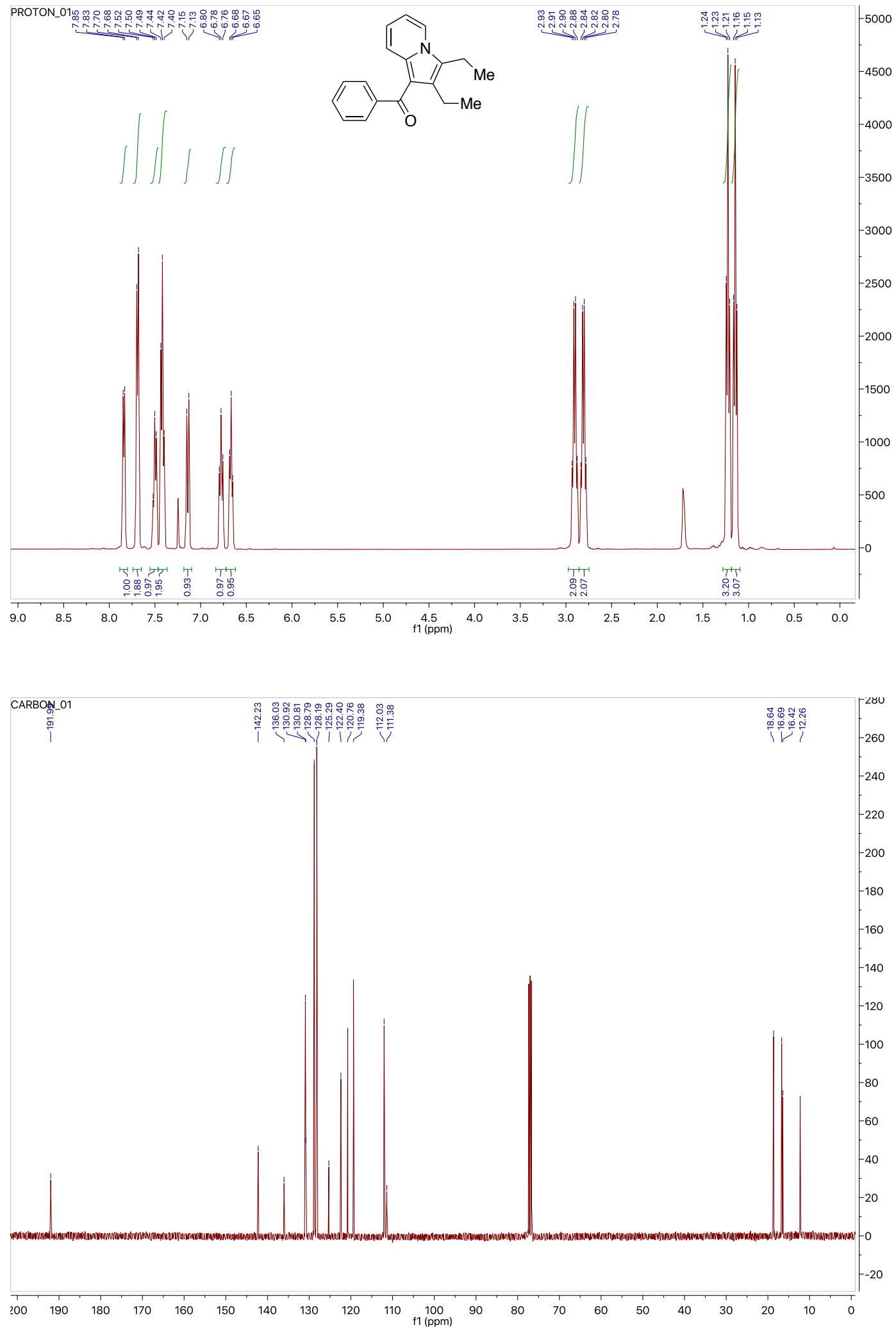
S60 Supporting Information. Rossler, Hartgerink, Zerull, Anderson et. al.

\section{Compound 9ff}
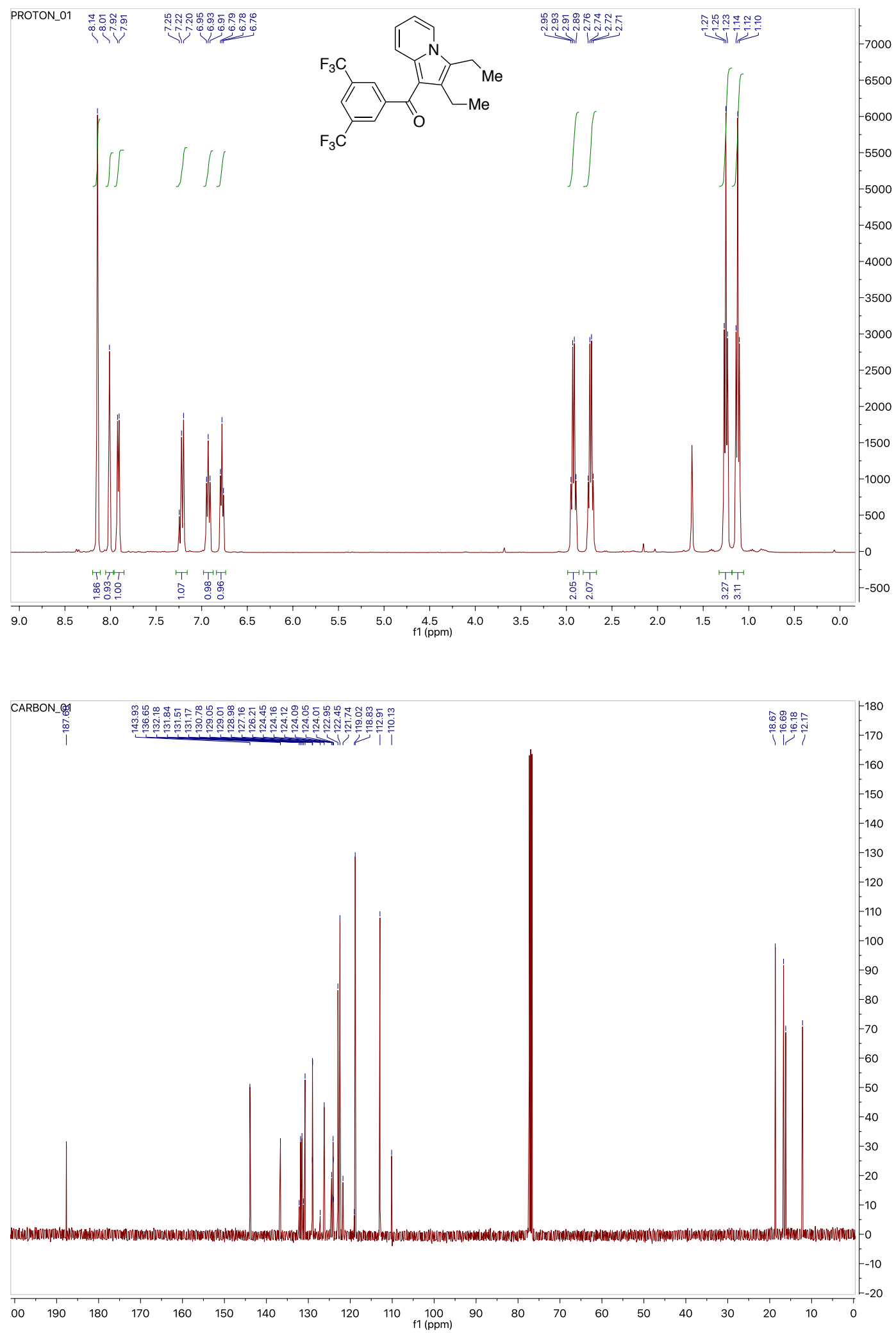
S61 Supporting Information. Rossler, Hartgerink, Zerull, Anderson et. al.

\section{Compound 9g}
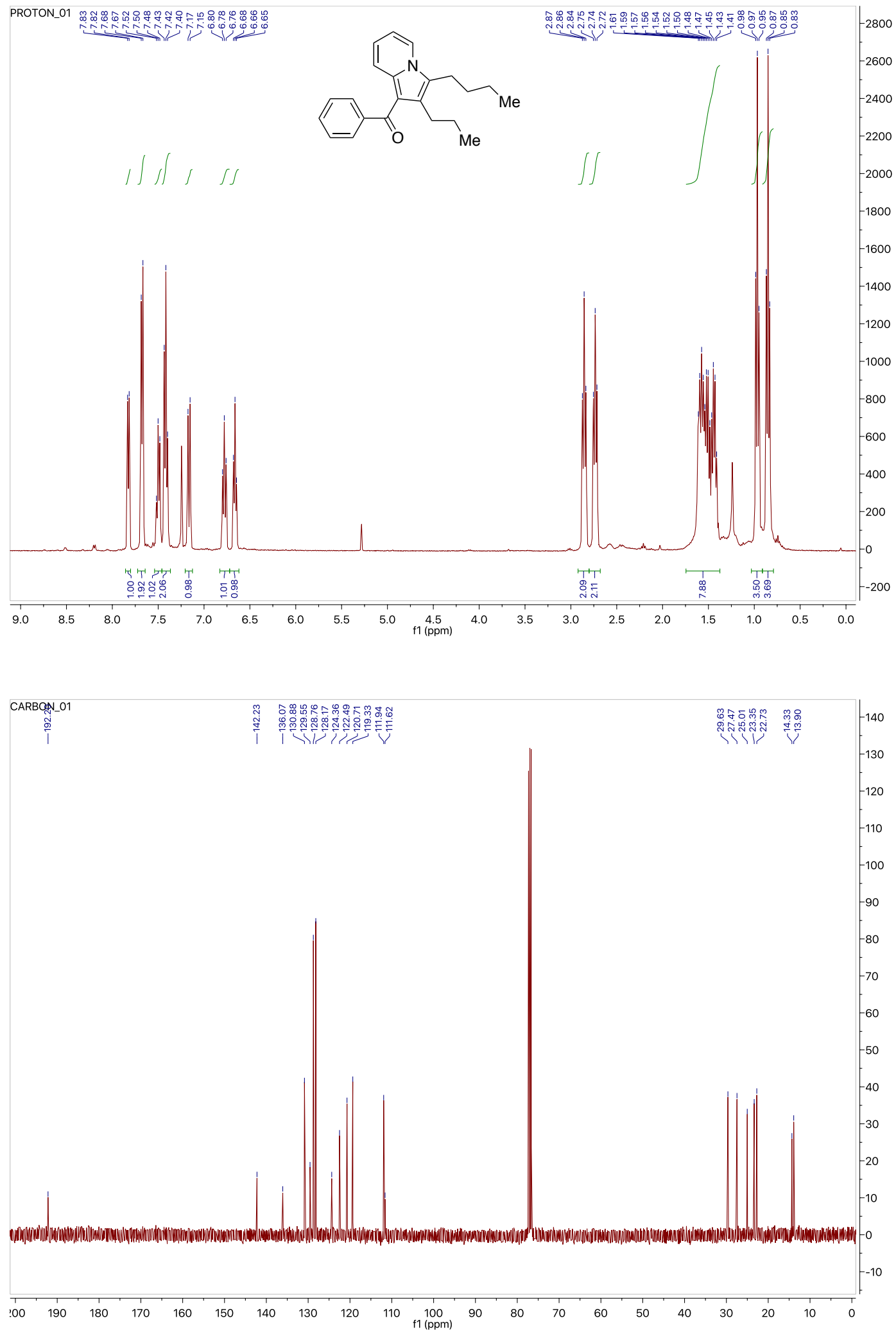
S62 Supporting Information. Rossler, Hartgerink, Zerull, Anderson et. al.

\section{Compound 11c}
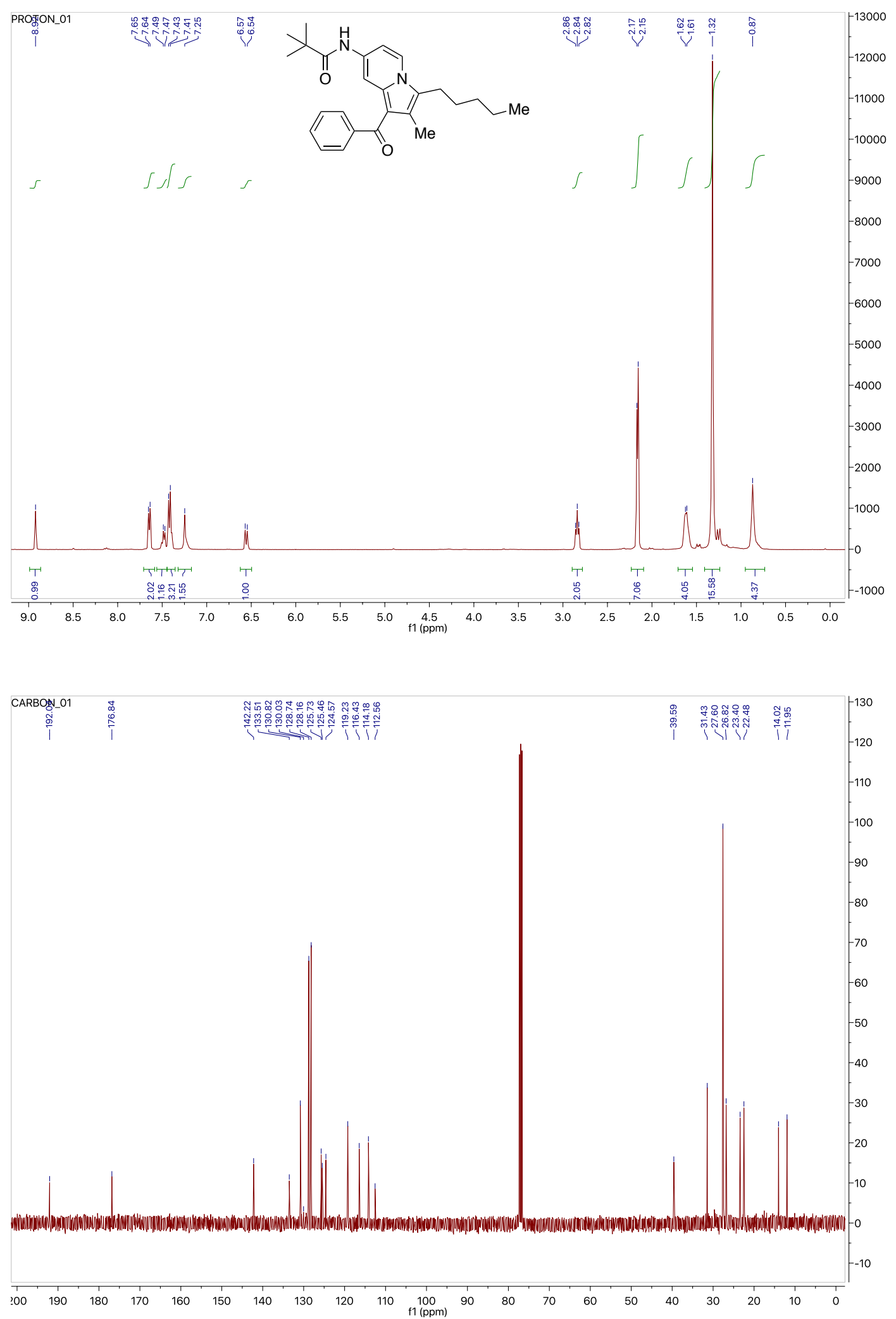
S63 Supporting Information. Rossler, Hartgerink, Zerull, Anderson et. al.

\section{Compound 11ce}

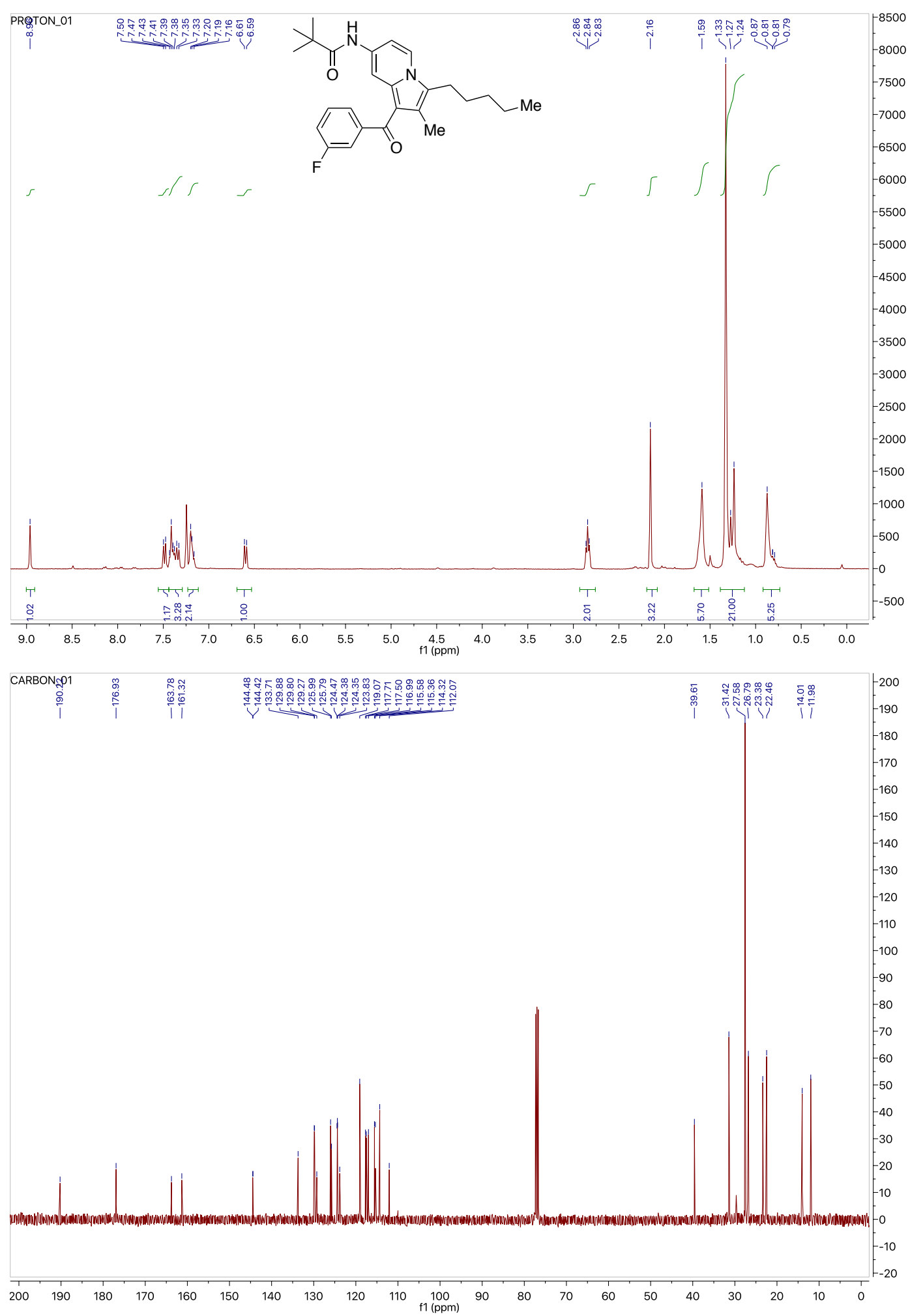


S64 Supporting Information. Rossler, Hartgerink, Zerull, Anderson et. al.

\section{Compound 11ecc}

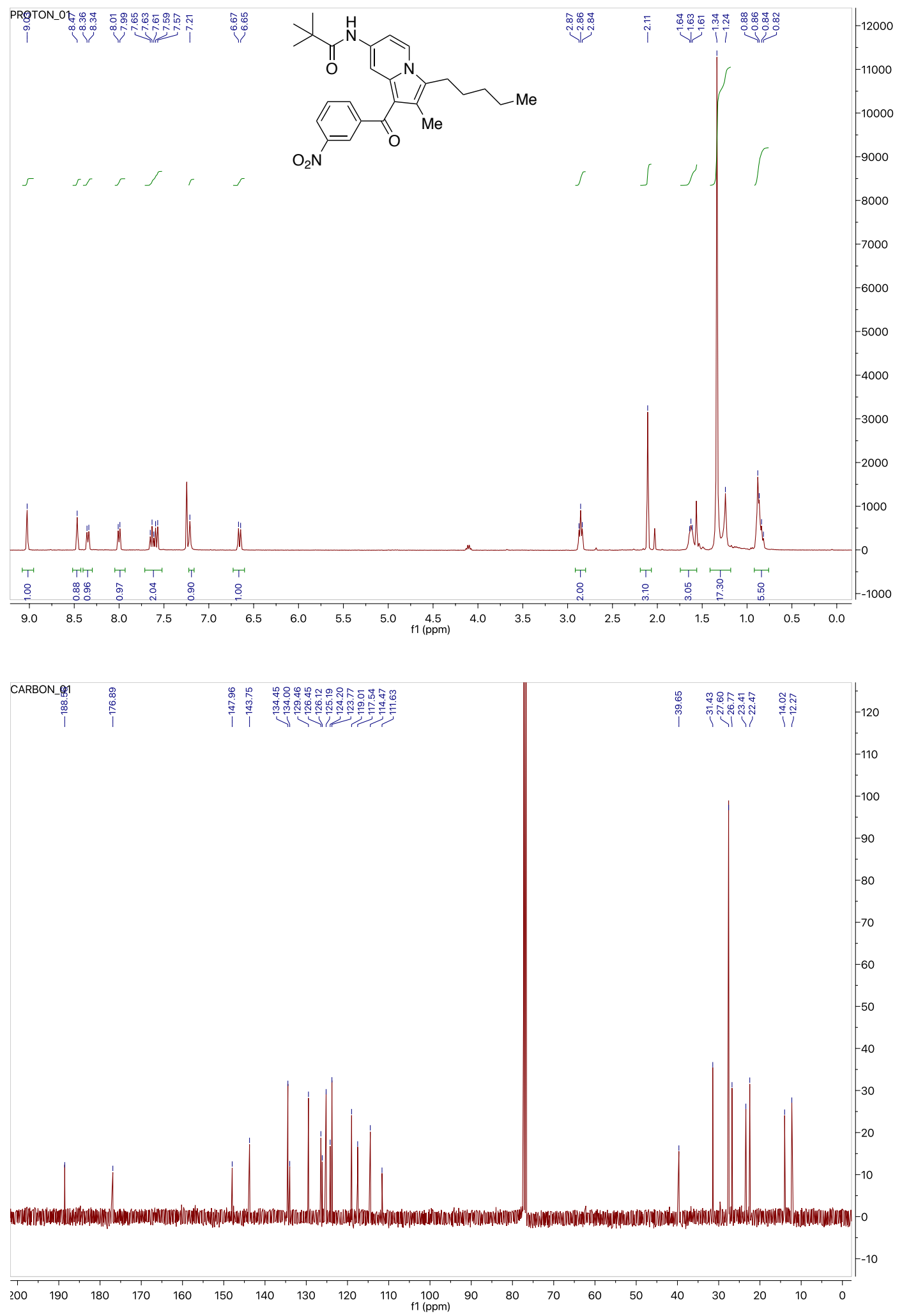


S65 Supporting Information. Rossler, Hartgerink, Zerull, Anderson et. al.

\section{Compound 11d}
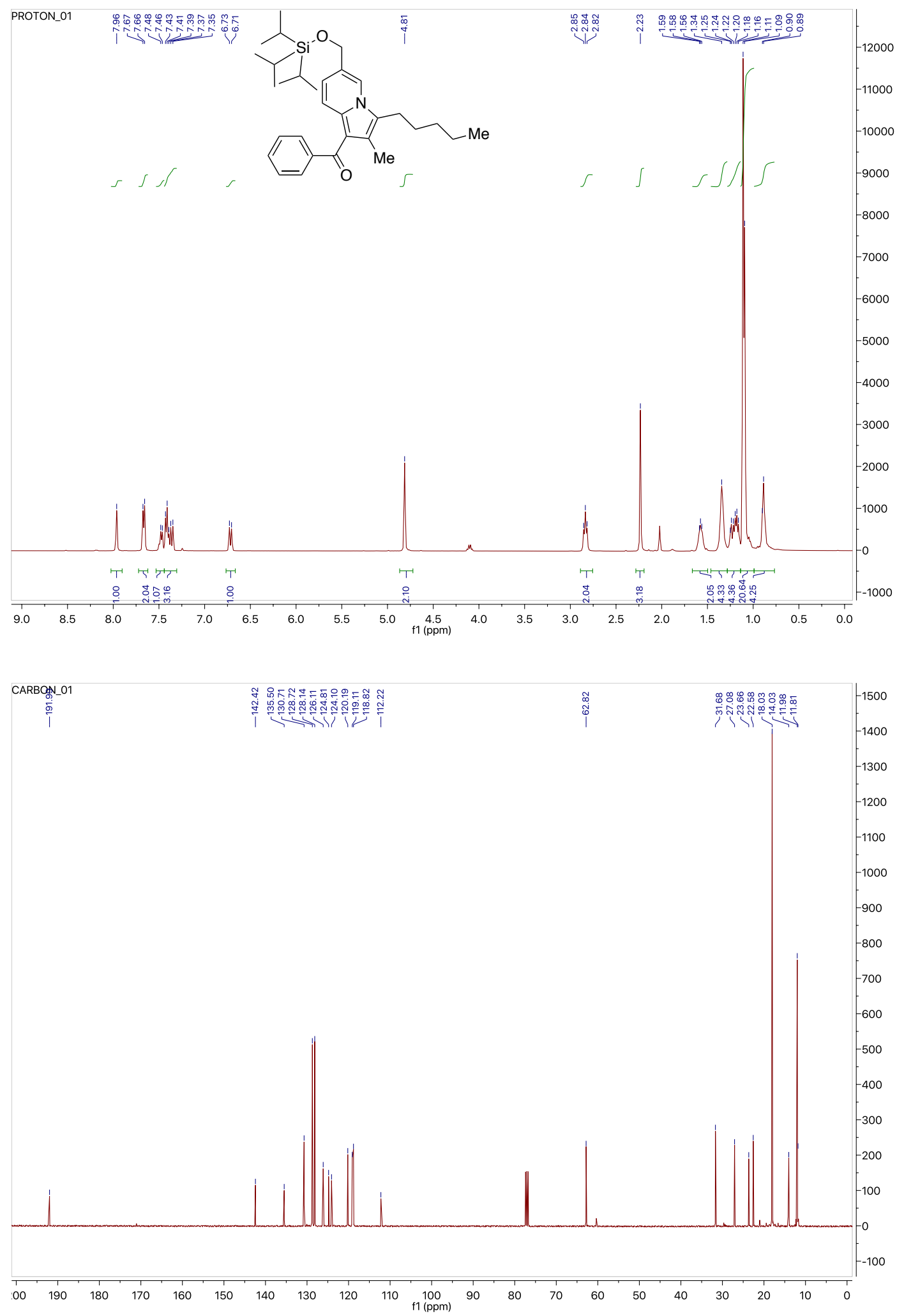
S66 Supporting Information. Rossler, Hartgerink, Zerull, Anderson et. al.

\section{Compound 11dd}
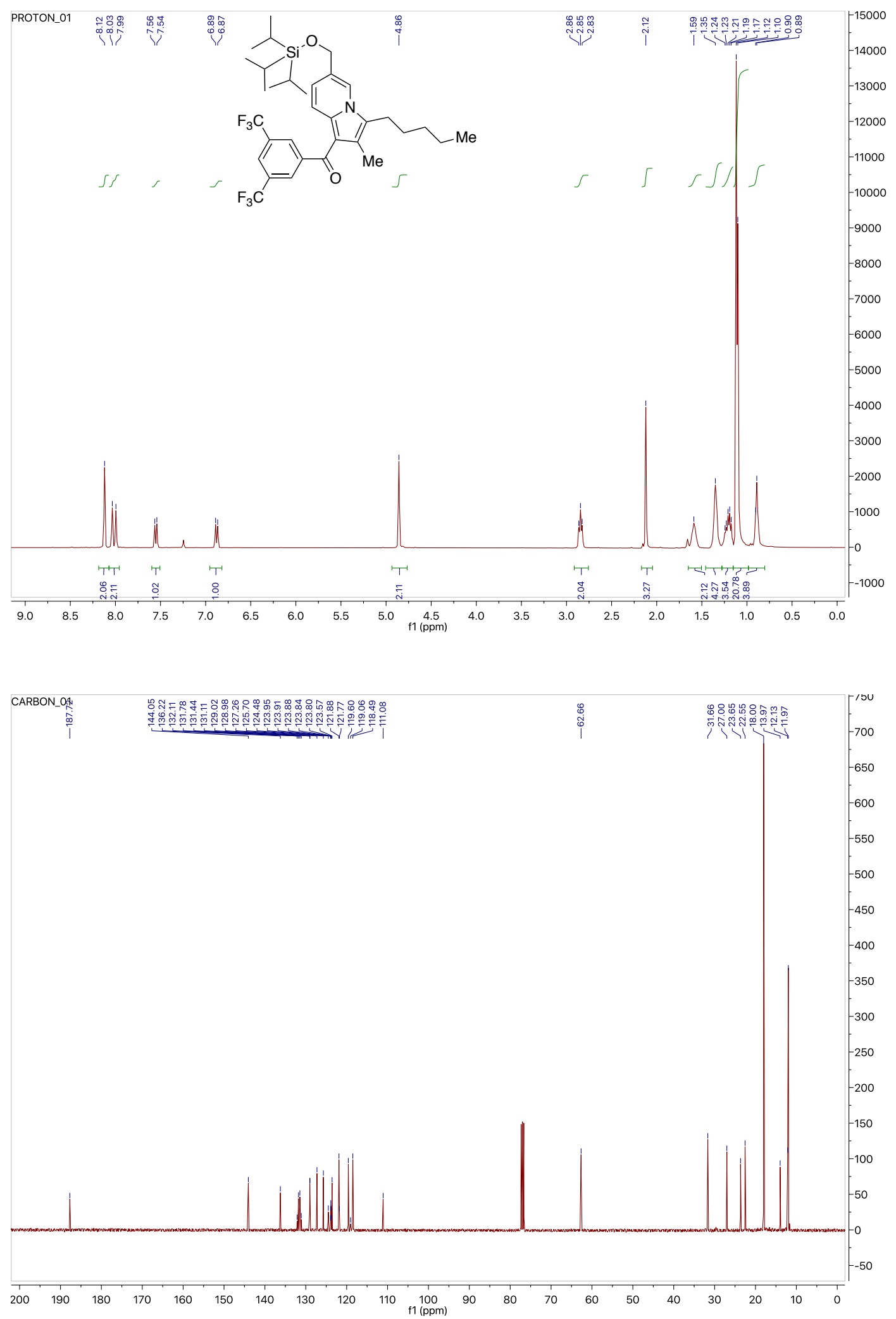
S67 Supporting Information. Rossler, Hartgerink, Zerull, Anderson et. al.

\section{Compound 11e}

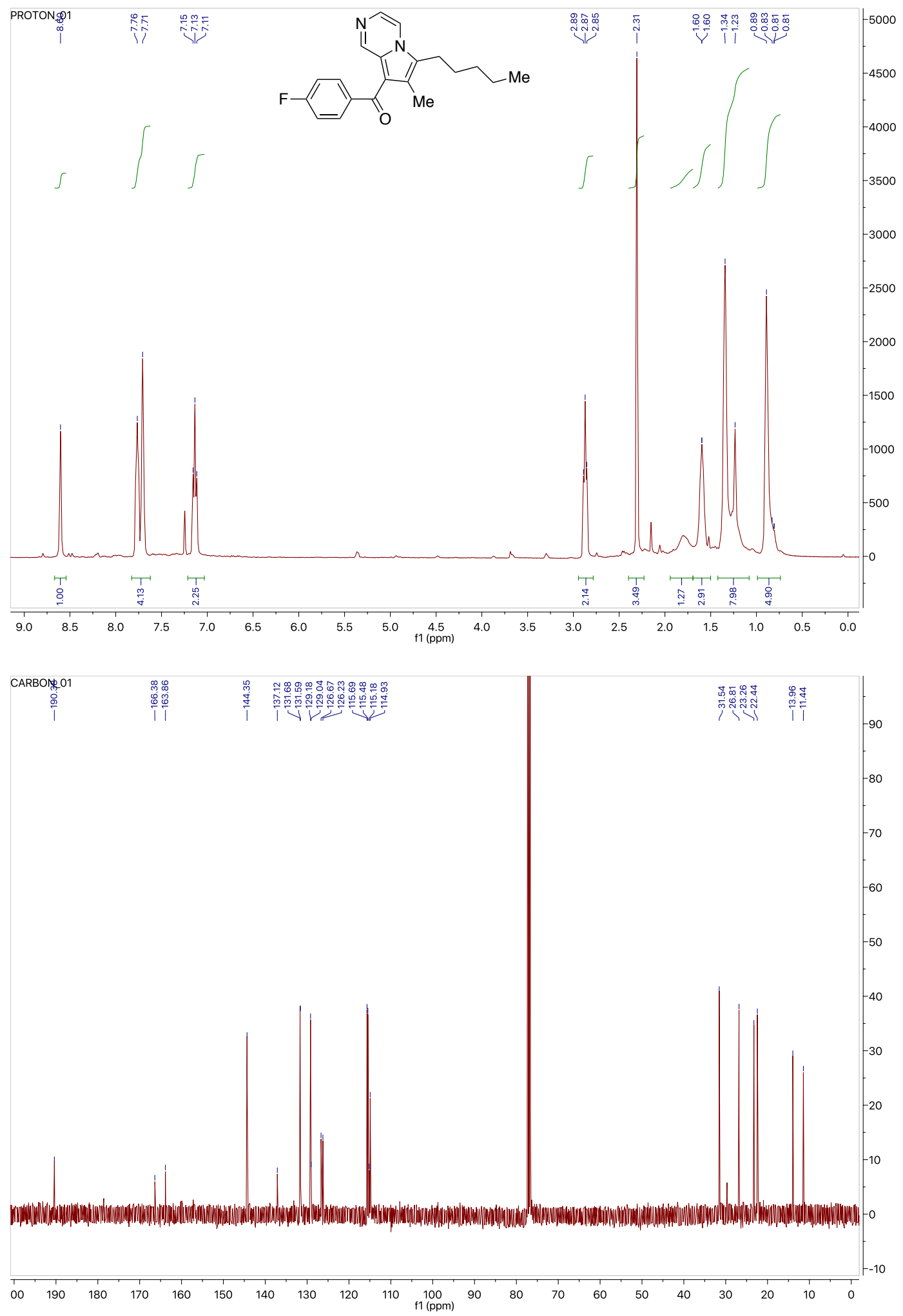


S68 Supporting Information. Rossler, Hartgerink, Zerull, Anderson et. al.

\section{Compound 11ee}

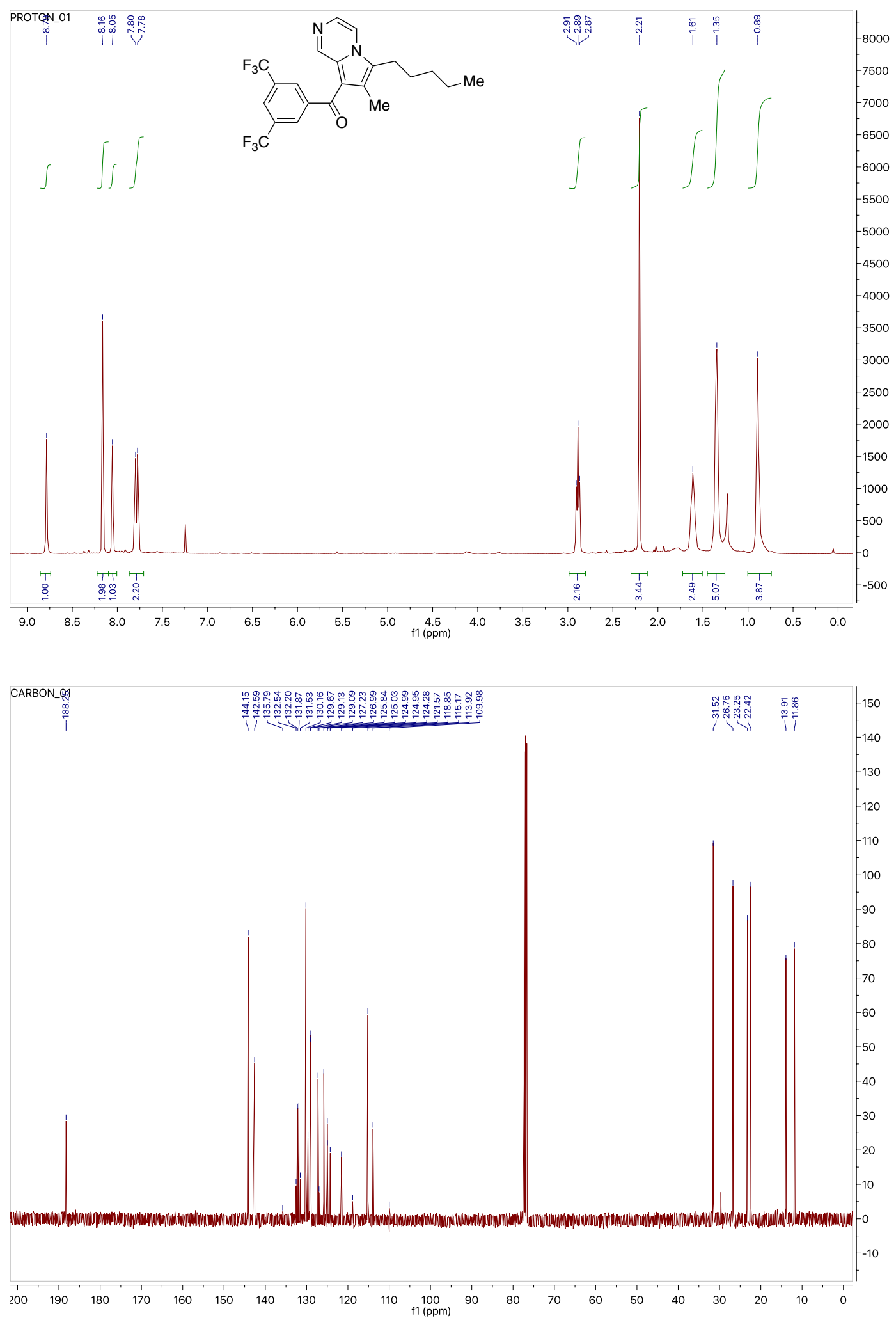


S69 Supporting Information. Rossler, Hartgerink, Zerull, Anderson et. al.

\section{Compound 13}
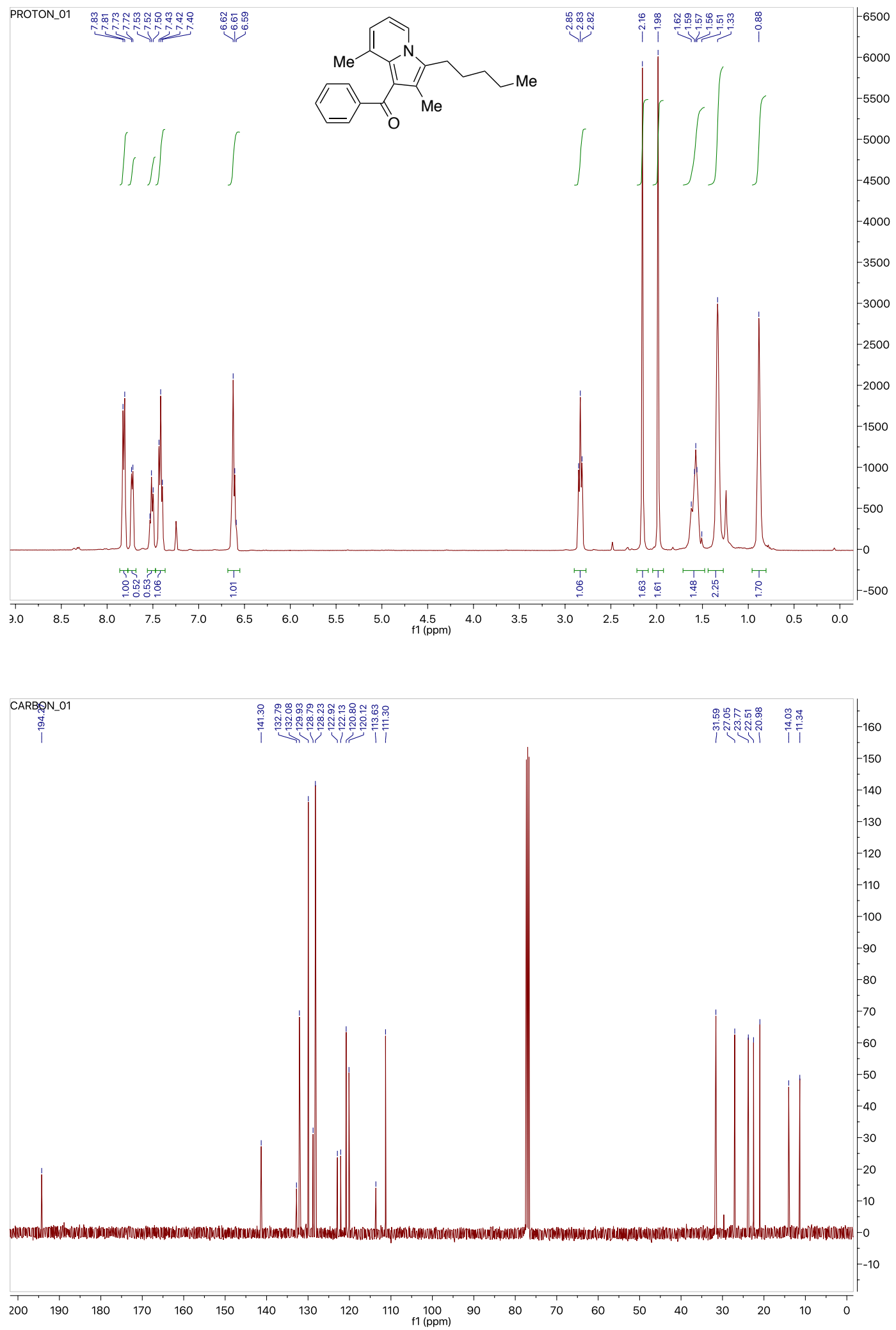
S70 Supporting Information. Rossler, Hartgerink, Zerull, Anderson et. al.

\section{Compound 13a}
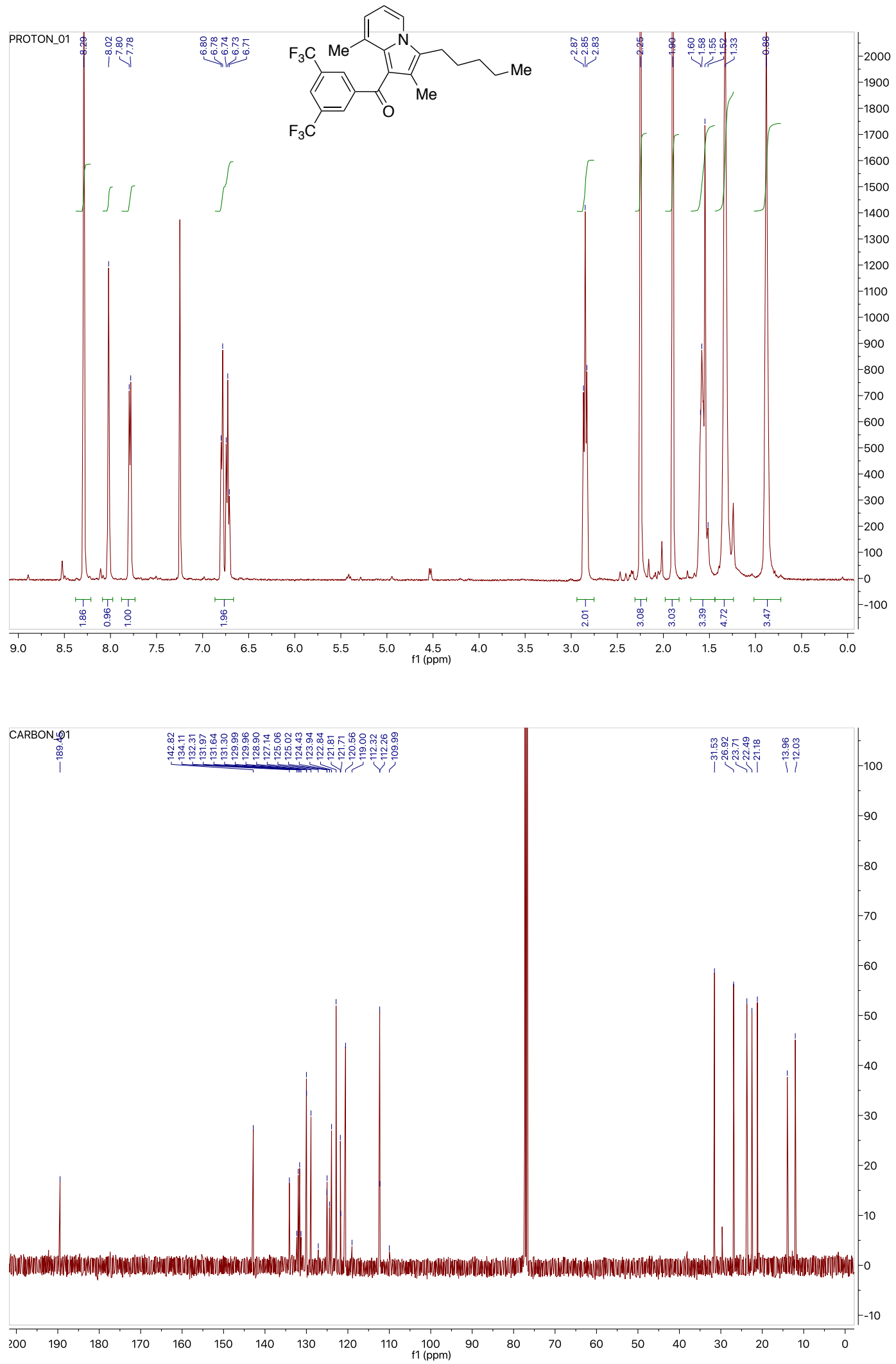
S71 Supporting Information. Rossler, Hartgerink, Zerull, Anderson, et. al.

\section{Compound 16}
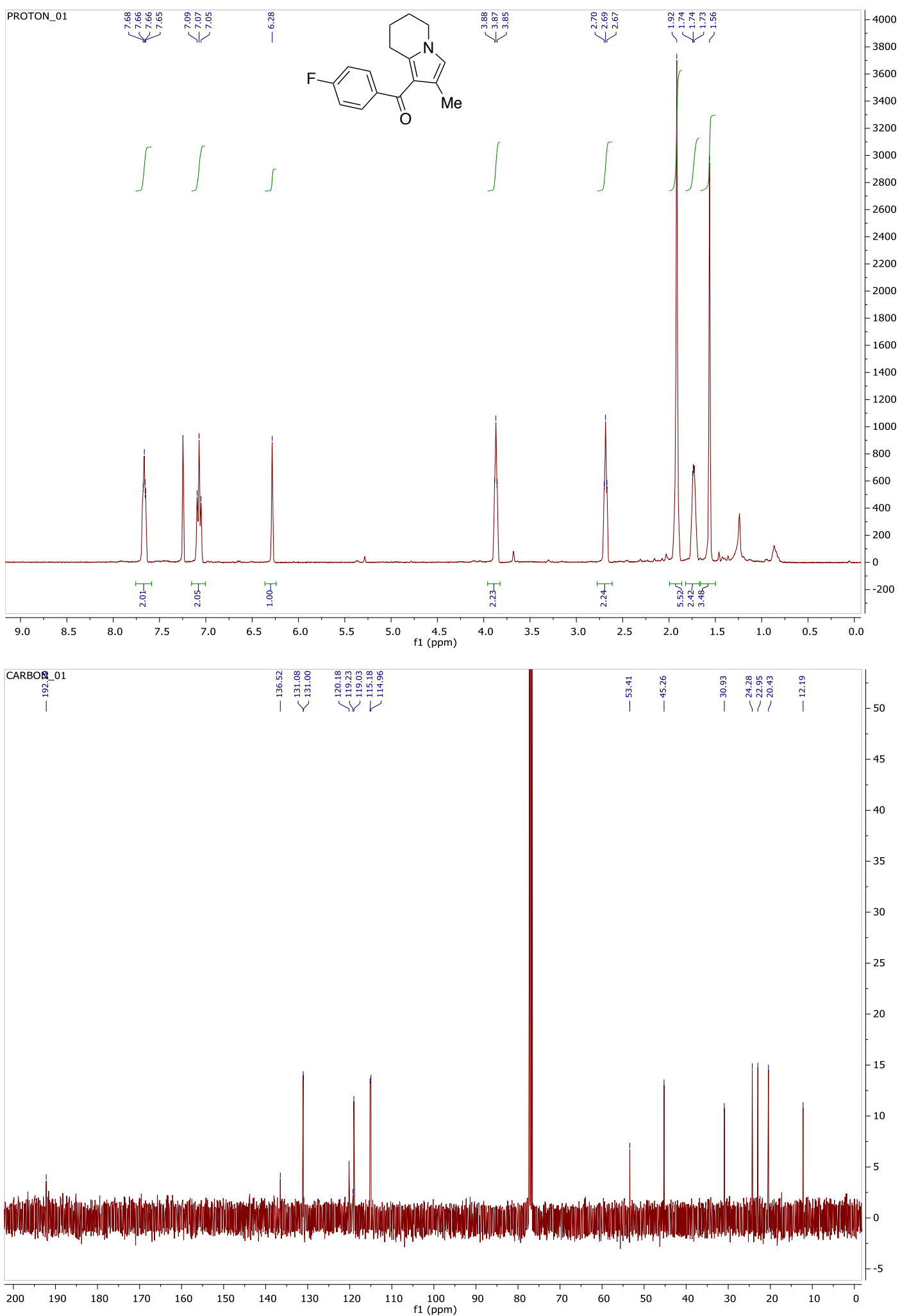